\title{
Synthesis of the Pyrrolidinone Core of KSM-2690 B
}

Timothy J. Donohoe, * Jessica Y. K. Chiu and Rhian E. Thomas ${ }^{\dagger}$ Chemistry Research Laboratory, University of Oxford, Mansfield Road, Oxford OXI 3TA, UK.

\section{Supporting Information}

timothy.donohoe@chem.ox.ac.uk 
Table of Contents

Materials and Methods $\quad 4$

Experimental 4

$N$-Boc pyrrole ester $8 \quad 4$

anti-Aldol $10 \quad 6$

BOM ether 21

syn-Diol 11

Iodohydrin 22

$\begin{array}{lr}\text { Hydroxyester } 12 & 10\end{array}$

$\begin{array}{ll}\text { Ketoester } \mathbf{1 3} & 11\end{array}$

Methylated ketoester $14 \quad 12$

Homoallyl alcohol 15

Allyl alcohol $16 \quad 14$

1,2-Diol 23

Acetonide $17 \quad 16$

$N$-Boc lactam $18 \quad 17$

$\begin{array}{lr}\text { Lactam } 24 & 18\end{array}$

$N$-methylated lactam $19 \quad 19$

1,3-Diol $20 \quad 19$

Hydroxyaldehyde $\mathbf{2 5} 20$

$\begin{array}{ll}\text { Lactone } 3 & 21\end{array}$

References $\quad 22$

Copies of NMR Spectra $\quad 21$

${ }^{13} \mathrm{C}$ NMR of $N$-Boc pyrrole 8

${ }^{13} \mathrm{C}$ NMR of anti-aldol $\mathbf{1 0}$

${ }^{1} \mathrm{H}$ NMR of BOM ether 21

${ }^{13} \mathrm{C}$ NMR of BOM ether $\mathbf{2 1} 26$

${ }^{1} \mathrm{H}$ NMR of syn-diol 11

${ }^{13} \mathrm{C}$ NMR of syn-diol 11

${ }^{1} \mathrm{H}$ NMR of iodohydrin 22

${ }^{13} \mathrm{C}$ NMR of iodohydrin 22

${ }^{1} \mathrm{H}$ NMR of hydroxyester $\mathbf{1 2} 31$

${ }^{13} \mathrm{C}$ NMR of hydroxyester $\mathbf{1 2} 32$

${ }^{1} \mathrm{H}$ NMR of ketoester $\mathbf{1 3} 33$

${ }^{13}$ C NMR of ketoester $\mathbf{1 3}$

${ }^{1} \mathrm{H}$ NMR of methylated ketoester $\mathbf{1 4}$ 
${ }^{13} \mathrm{C}$ NMR of methylated ketoester $\mathbf{1 4} 36$

${ }^{1} \mathrm{H}$ NMR of homoallyl alcohol $\mathbf{1 5} 37$

${ }^{13} \mathrm{C}$ NMR of homoallyl alcohol $\mathbf{1 5} 38$

${ }^{1} \mathrm{H}$ NMR of allyl alcohol $\mathbf{1 6}$

${ }^{13} \mathrm{C}$ NMR of allyl alcohol $16 \quad 40$

${ }^{1} \mathrm{H}$ NMR of 1,2-diol 23

${ }^{13}$ C NMR of 1,2-diol 23

${ }^{1} \mathrm{H}$ NMR of acetonide $\mathbf{1 7} 43$

${ }^{13} \mathrm{C}$ NMR of acetonide $\mathbf{1 7} 44$

${ }^{1} \mathrm{H}$ NMR of $N$-Boc lactam 18

${ }^{13} \mathrm{C}$ NMR of $N$-Boc lactam 18

${ }^{1} \mathrm{H}$ NMR of lactam 24

${ }^{13} \mathrm{C}$ NMR of lactam 24

${ }^{1} \mathrm{H}$ NMR of $N$-methylated lactam 19

${ }^{13} \mathrm{C}$ NMR of $N$-methylated lactam 19

${ }^{1}$ H NMR of 1,3-diol 20

${ }^{13} \mathrm{C}$ NMR of 1,3-diol 20

${ }^{1} \mathrm{H}$ NMR of hydroxyaldehyde $\mathbf{2 5} 53$

${ }^{13} \mathrm{C}$ NMR of hydroxyaldehyde $\mathbf{2 5} 54$

${ }^{1} \mathrm{H}$ NMR of lactone 3

${ }^{13} \mathrm{C}$ NMR of lactone 3 


\section{Materials and Methods}

All solvents and reagents requiring purification were purified using standard laboratory techniques according to methods published by Perrin, Armarego, and Perrin (Pergamon Press, 1966) apart from $\mathrm{CH}_{2} \mathrm{Cl}_{2}$, THF and $\mathrm{Et}_{2} \mathrm{O}$ which were dried by filtration through an activated alumina purification column. Petrol refers to petroleum ether in the boiling range $40-60{ }^{\circ} \mathrm{C}$. All reactions sensitive to oxygen or moisture were conducted under an argon atmosphere. Reactions were monitored by thin layer chromatography (TLC) using Merck Kieselgel $60 \mathrm{~F}_{254}$ $250 \mu \mathrm{m}$ pre-coated aluminium plates. Flash chromatography was performed using silica gel 60 (33-70 $\mu \mathrm{m}, \mathrm{BDH})$.

${ }^{1} \mathrm{H}$ NMR and ${ }^{13} \mathrm{C}$ NMR spectra were obtained on a Bruker Avance AV400, or on a Bruker Avance AV500 with cryoprobe spectrometer. Chemical shifts are reported in $\delta$ values relative to an internal standard of tetramethylsilane (TMS). Infrared (IR) spectra were recorded on a Bruker Tensor 27 FTIR spectrometer, as evaporated films or $\mathrm{KBr}$ discs. Electrospray ionisation (ESI) was performed on a Fisons Platform using tetraoctylammoniun bromide as the lock mass. High-resolution mass spectra were recorded on a Bruker MicroTOF spectrometer.

\section{Experimental}

\section{$N$-Boc pyrrole ester $8^{1}$}

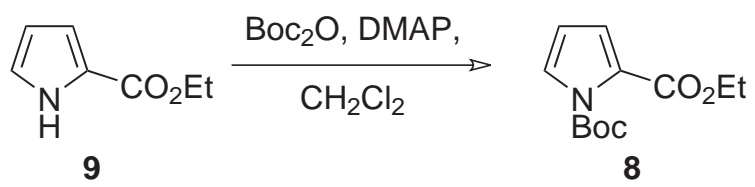

A solution of di-tert-butyl dicarbonate $(16.1 \mathrm{~g}, 73.7 \mathrm{mmol})$ in $\mathrm{CH}_{2} \mathrm{Cl}_{2}(20 \mathrm{~mL})$ was added dropwise via cannula to a solution of ethyl $1 H$-pyrrole-2-carboxylate $(9.31 \mathrm{~g}, 67.0 \mathrm{mmol})$ and DMAP (819 mg, $6.70 \mathrm{mmol})$ in $\mathrm{CH}_{2} \mathrm{Cl}_{2}(40 \mathrm{~mL})$. The reaction mixture was stirred at $\mathrm{rt}$ for $20 \mathrm{~h}$. Aqueous $1 \mathrm{M} \mathrm{HCl}(100 \mathrm{~mL})$ was added and the layers were separated. The aqueous layer was extracted with $\mathrm{Et}_{2} \mathrm{O}(3 \times 50 \mathrm{~mL})$ and the combined organic layers were dried with $\mathrm{MgSO}_{4}$, filtered and evaporated under reduced pressure. The crude product was purified by flash column chromatography (5\% EtOAc-petrol) to give $N$-Boc ethyl ester pyrrole 8 (15.8 g, $66.2 \mathrm{mmol}, 99 \%$ ) as a pale yellow oil.

${ }^{1} \mathbf{H}$ NMR $\left(400 \mathrm{MHz}, \mathrm{CDCl}_{3}\right) \delta 7.29(\mathrm{dd}, 1 \mathrm{H}, J=3.3$ and $1.7 \mathrm{~Hz}), 6.81(\mathrm{dd}, 1 \mathrm{H}, J=3.3$ and $1.7 \mathrm{~Hz}), 6.14(\mathrm{t}, 1 \mathrm{H}, J=3.3 \mathrm{~Hz}), 4.28(\mathrm{q}, 2 \mathrm{H}, J=7.1 \mathrm{~Hz}), 1.56(\mathrm{~s}, 9 \mathrm{H})$ and $1.33(\mathrm{t}, 3 \mathrm{H}$, 
$J=7.1 \mathrm{~Hz}) ;{ }^{13} \mathrm{C}$ NMR $\left(100 \mathrm{MHz}, \mathrm{CDCl}_{3}\right) \delta 160.7(\mathrm{C}), 148.3(\mathrm{C}), 126.4(\mathrm{CH}), 125.5(\mathrm{C})$, $120.5(\mathrm{CH}), 110.0(\mathrm{CH}), 84.5(\mathrm{C}), 60.6\left(\mathrm{CH}_{2}\right), 27.5\left(3 \times \mathrm{CH}_{3}\right)$ and $14.3\left(\mathrm{CH}_{3}\right)$. The data was consistent with that previously reported in the literature. ${ }^{1}$ 
anti-Aldol $10^{2}$

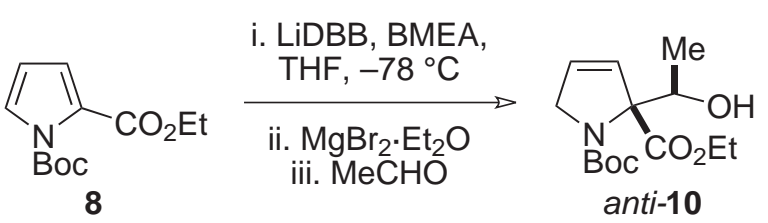

Freshly cut lithium wire $(216 \mathrm{mg}, 30.9 \mathrm{mmol}$ ) was hammered out into a foil, cut into thin strips and placed in a Schlenk tube containing DBB (7.98 g, $35.2 \mathrm{mmol}$ ) and glass "antibumping granules". The Schlenk tube was evacuated and purged with argon several times. The contents of the Schlenk tube were stirred vigorously until the lithium foil had been completely reduced to powder $(\sim 18 \mathrm{~h})$. THF $(60 \mathrm{~mL})$ was added, resulting in a dark turquoise solution which was cooled to $-78^{\circ} \mathrm{C}$. A solution of $N$-Boc pyrrole 8 (2.00 g, $\left.8.35 \mathrm{mmol}\right)$ and BMEA $(1.5 \mathrm{~mL}, 10.7 \mathrm{mmol})$ in THF $(35 \mathrm{~mL})$ was added dropwise via cannula. The turquoise colour persisted throughout the course of the substrate addition. The reaction mixture was stirred at $-78{ }^{\circ} \mathrm{C}$ for $15 \mathrm{~min}$ and 1,2-dibromoethane was added dropwise until the turquoise colour disappeared. $\mathrm{MgBr}_{2} \cdot \mathrm{Et}_{2} \mathrm{O}$ (2.38 g, $9.20 \mathrm{mmol}$ ) was added and the solution was stirred rapidly for $30 \mathrm{~min}$. Acetaldehyde $(1.3 \mathrm{~mL}, 23.2 \mathrm{mmol})$ was added and the reaction mixture was stirred for a further 20 min before quenching with saturated $\mathrm{NH}_{4} \mathrm{Cl}(15 \mathrm{~mL})$. The reaction mixture was warmed to $\mathrm{rt}$ and poured into aqueous $1 \mathrm{M} \mathrm{HCl}(50 \mathrm{~mL})$ and $\mathrm{Et}_{2} \mathrm{O}(50 \mathrm{~mL})$. The layers were separated and the aqueous layer was extracted with $\mathrm{Et}_{2} \mathrm{O}(3 \times 50 \mathrm{~mL})$. The combined extracts were dried with $\mathrm{MgSO}_{4}$, filtered and evaporated under reduced pressure. The crude product was purified by gradient flash column chromatography $(2.5 \% \rightarrow 4 \%$ $\mathrm{Me}_{2} \mathrm{CO}$-petrol) to give anti-aldol 10 (1.33 g, $\left.4.61 \mathrm{mmol}, 56 \%\right)$ and syn-aldol 10 (340 mg, $1.19 \mathrm{mmol}, 14 \%$ ) as pale yellow oils.

Data for anti-aldol 10:

${ }^{1} \mathbf{H}$ NMR $\left(400 \mathrm{MHz}, \mathrm{CDCl}_{3}\right)(\sim 1.4: 1$ mixture of Boc rotamers) (major Boc rotamer) $\delta 6.12$ $(\mathrm{dt}, 1 \mathrm{H}, J=6.4$ and $2.0 \mathrm{~Hz}), 5.81(\mathrm{dt}, 1 \mathrm{H}, J=6.4$ and $2.4 \mathrm{~Hz}), 4.68(\mathrm{q}, 1 \mathrm{H}, J=6.4 \mathrm{~Hz}), 4.41-$ $4.06(\mathrm{~m}, 4 \mathrm{H}), 3.84(\mathrm{br} \mathrm{s}, 1 \mathrm{H}), 1.43(\mathrm{~s}, 9 \mathrm{H}), 1.27(\mathrm{t}, 3 \mathrm{H}, J=7.2 \mathrm{~Hz})$ and $1.02(\mathrm{~d}, 3 \mathrm{H}$, $J=6.4 \mathrm{~Hz})$; (minor Boc rotamer) $\delta 6.04(\mathrm{dt}, 1 \mathrm{H}, J=6.4$ and $1.6 \mathrm{~Hz}), 5.83(\mathrm{dt}, 1 \mathrm{H}, J 6.4$ and $2.0 \mathrm{~Hz}), 4.74(\mathrm{q}, 1 \mathrm{H}, J=6.4 \mathrm{~Hz}), 4.41-4.06(\mathrm{~m}, 5 \mathrm{H}), 1.46(\mathrm{~s}, 9 \mathrm{H}), 1.24(\mathrm{t}, 3 \mathrm{H}, J=7.2 \mathrm{~Hz})$ and $1.06(\mathrm{~d}, 3 \mathrm{H}, J=6.4 \mathrm{~Hz}),{ }^{13} \mathrm{C}$ NMR $\left(100 \mathrm{MHz}, \mathrm{CDCl}_{3}\right)$ (major Boc rotamer) $174.0(\mathrm{C}), 152.7$ (C), $129.9(\mathrm{CH}), 126.2(\mathrm{CH}), 80.6(\mathrm{C}), 77.4(\mathrm{C}), 68.9(\mathrm{CH}), 61.6\left(\mathrm{CH}_{2}\right), 55.2\left(\mathrm{CH}_{2}\right), 28.2$ $\left(3 \times \mathrm{CH}_{3}\right), 15.6\left(\mathrm{CH}_{3}\right)$ and $13.9\left(\mathrm{CH}_{3}\right)$; (minor Boc rotamer) $\delta 173.4(\mathrm{C}), 153.7(\mathrm{C}), 129.6$ $(\mathrm{CH}), 126.4(\mathrm{CH}), 80.2(\mathrm{C}), 78.2(\mathrm{C}), 68.7(\mathrm{CH}), 61.4\left(\mathrm{CH}_{2}\right), 55.2\left(\mathrm{CH}_{2}\right), 28.3\left(3 \times \mathrm{CH}_{3}\right), 16.1$ $\left(\mathrm{CH}_{3}\right)$ and $14.0\left(\mathrm{CH}_{3}\right)$. The data was consistent with that previously reported in the literature. ${ }^{2}$ 


\section{BOM ether 21}

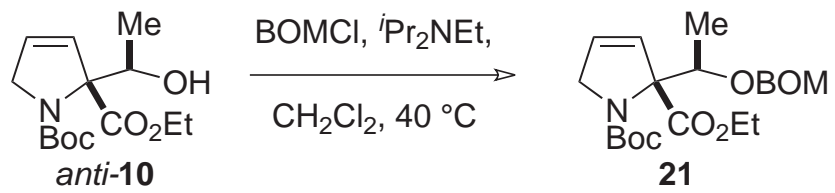

$N$-Ethyldiisopropylamine $(15 \mathrm{~mL}, 86.1 \mathrm{mmol})$ was added to a stirred solution of anti-aldol $\mathbf{1 0}$ (4.87 g, $17.1 \mathrm{mmol})$ in $\mathrm{CH}_{2} \mathrm{Cl}_{2}(100 \mathrm{~mL})$. The solution was cooled to $0{ }^{\circ} \mathrm{C}$ and $\mathrm{BOMCl}$ $(7.3 \mathrm{~mL}, 52.5 \mathrm{mmol})$ was added dropwise. The reaction mixture was stirred at $40{ }^{\circ} \mathrm{C}$ until TLC analysis showed the reaction was complete $(2 \mathrm{~d})$. The reaction was quenched with saturated $\mathrm{NH}_{4} \mathrm{Cl}(25 \mathrm{~mL})$ and the layers were separated. The aqueous layer was extracted with $\mathrm{Et}_{2} \mathrm{O}(3 \times 80 \mathrm{~mL})$ and the combined organic extracts were dried with $\mathrm{MgSO}_{4}$ and evaporated under reduced pressure. The crude product was purified by gradient flash column chromatography $\left(8 \% \rightarrow 16 \% \mathrm{Et}_{2} \mathrm{O}\right.$-petrol) to give $\mathrm{BOM}$ ether $21(6.49 \mathrm{~g}, 16.0 \mathrm{mmol}, 94 \%)$ as a pale yellow oil.

$\boldsymbol{R}_{f}\left(20 \% \mathrm{Me}_{2} \mathrm{CO}\right.$-petrol) 0.32; IR (thin film, $\mathrm{cm}^{-1}$ ) 2979, 1744, 1705, 1455, 1394, 1329, 1250, 1217, 1152, 1107, 1039; ${ }^{1} \mathbf{H}$ NMR $\left(400 \mathrm{MHz}, \mathrm{CDCl}_{3}\right)$ ( 1.8:1 mixture of Boc rotamers) (major Boc rotamer) $\delta 7.38-7.23(\mathrm{~m}, 5 \mathrm{H}), 6.09(\mathrm{~d}, 1 \mathrm{H}, J=6.3 \mathrm{~Hz}), 5.78(\mathrm{~d}, 1 \mathrm{H}$, $J=6.3 \mathrm{~Hz}), 5.08(\mathrm{~d}, 1 \mathrm{H}, J=7.0 \mathrm{~Hz}), 4.86(\mathrm{~d}, 1 \mathrm{H}, J=7.0 \mathrm{~Hz}), 4.73(\mathrm{~d}, 1 \mathrm{H}, J=11.5 \mathrm{~Hz}), 4.61$ (q, 1H, $J=6.3 \mathrm{~Hz}), 4.55(\mathrm{~d}, 1 \mathrm{H}, J=11.5 \mathrm{~Hz}), 4.39-4.02(\mathrm{~m}, 4 \mathrm{H}), 1.42(\mathrm{~s}, 9 \mathrm{H}), 1.23(\mathrm{t}, 3 \mathrm{H}$, $J=7.2 \mathrm{~Hz})$ and $1.11(\mathrm{~d}, 3 \mathrm{H}, J=6.3 \mathrm{~Hz}) ;($ minor Boc rotamer $) \delta 7.38-7.23(\mathrm{~m}, 5 \mathrm{H}), 6.04(\mathrm{~d}$, $1 \mathrm{H}, J=6.3 \mathrm{~Hz}), 5.81(\mathrm{~d}, 1 \mathrm{H}, J=6.3 \mathrm{~Hz}), 5.11(\mathrm{dd}, 1 \mathrm{H}, J=7.0 \mathrm{~Hz}), 4.86(\mathrm{~d}, 1 \mathrm{H}, J=7.0 \mathrm{~Hz})$, $4.77(\mathrm{q}, 1 \mathrm{H}, J=6.3 \mathrm{~Hz}), 4.73(\mathrm{~d}, 1 \mathrm{H}, J=11.5 \mathrm{~Hz}), 4.53(\mathrm{~d}, 1 \mathrm{H}, J=11.5 \mathrm{~Hz}), 4.39-4.02(\mathrm{~m}$, $4 \mathrm{H}), 1.44(\mathrm{~s}, 9 \mathrm{H}), 1.21(\mathrm{t}, 3 \mathrm{H}, J=7.6 \mathrm{~Hz})$ and $1.11(\mathrm{~d}, 3 \mathrm{H}, J=6.3 \mathrm{~Hz}) ;{ }^{13} \mathbf{C}$ NMR $(100 \mathrm{MHz}$, $\mathrm{CDCl}_{3}$ ) (major Boc rotamer) $\delta 171.2(\mathrm{C}), 152.8(\mathrm{C}), 138.0(\mathrm{C}), 129.1(\mathrm{CH}), 128.4(2 \times \mathrm{CH})$, $127.9(2 \times \mathrm{CH}), 127.6(\mathrm{CH}), 127.4(\mathrm{CH}), 96.0\left(\mathrm{CH}_{2}\right), 80.8(\mathrm{C}), 77.2(\mathrm{C}), 76.8(\mathrm{CH}), 69.7$ $\left(\mathrm{CH}_{2}\right), 61.1\left(\mathrm{CH}_{2}\right), 55.2\left(\mathrm{CH}_{2}\right), 28.2\left(3 \times \mathrm{CH}_{3}\right), 16.0\left(\mathrm{CH}_{3}\right)$ and $14.1\left(\mathrm{CH}_{3}\right)$; (minor Boc rotamer) $\delta 171.2(\mathrm{C}), 153.4(\mathrm{C}), 138.2(\mathrm{C}), 129.2(\mathrm{CH}), 128.3(2 \times \mathrm{CH}), 127.9(2 \times \mathrm{CH})$, $127.6(\mathrm{CH}), 127.4(\mathrm{CH}), 96.2\left(\mathrm{CH}_{2}\right), 80.1(\mathrm{C}), 77.6(\mathrm{C}), 76.2(\mathrm{CH}), 69.5\left(\mathrm{CH}_{2}\right), 61.0\left(\mathrm{CH}_{2}\right)$, $55.3\left(\mathrm{CH}_{2}\right), 28.3\left(3 \times \mathrm{CH}_{3}\right), 16.4\left(\mathrm{CH}_{3}\right)$ and $14.2\left(\mathrm{CH}_{3}\right)$; LRMS (ESI, $\left.m / z\right) 464\left(\mathrm{M}+\mathrm{NH}_{4}{ }^{+}+\right.$ MeCN, 100\%); HRMS (ESI) calcd for $\mathrm{C}_{22} \mathrm{H}_{31} \mathrm{NO}_{6} \mathrm{Na}\left(\mathrm{M}+\mathrm{Na}^{+}\right) 428.2049$; found 428.2046 $(\triangle 0.8 \mathrm{ppm})$. 
syn-Diol 11

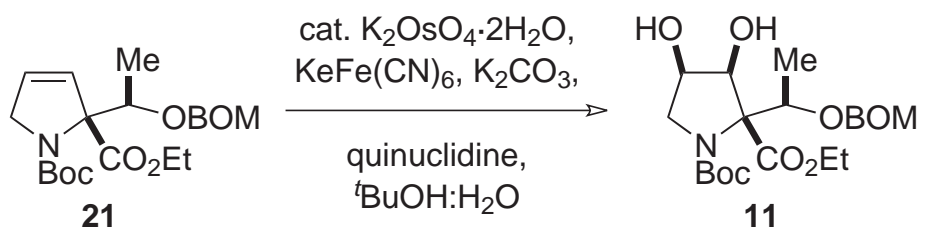

$\mathrm{K}_{3} \mathrm{Fe}(\mathrm{CN})_{6}(15.9 \mathrm{~g}, 48.3 \mathrm{mmol}), \mathrm{K}_{2} \mathrm{CO}_{3}(6.67 \mathrm{~g}, 48.3 \mathrm{mmol})$ and quinuclidine $(53.7 \mathrm{mg}$, $0.483 \mathrm{mmol})$ were added to BOM ether $21(6.53 \mathrm{~g}, 16.1 \mathrm{mmol})$ followed by a 1:1 mixture of ${ }^{t} \mathrm{BuOH}: \mathrm{H}_{2} \mathrm{O}(100 \mathrm{~mL}) . \mathrm{K}_{2} \mathrm{OsO}_{4} \cdot 2 \mathrm{H}_{2} \mathrm{O}(178 \mathrm{mg}, 0.483 \mathrm{mmol})$ was added in one portion. The resulting mixture was stirred for $36 \mathrm{~h}$ and saturated $\mathrm{Na}_{2} \mathrm{SO}_{3}(50 \mathrm{~mL})$ and EtOAc $(50 \mathrm{~mL})$ were added. The layers were separated and the aqueous layer was extracted with ethyl acetate $(3 \times 80 \mathrm{~mL})$. The combined organic layers were dried with $\mathrm{MgSO}_{4}$, filtered and evaporated under reduced pressure. The crude product was purified by flash column chromatography $\left(16 \% \rightarrow 25 \% \mathrm{Me}_{2} \mathrm{CO}\right.$-petrol) to give recovered BOM ether 21 (358 mg, $\left.0.884 \mathrm{mmol}, 5 \%\right)$, as well as syn-diol 11 (6.37 g, $14.5 \mathrm{mmol}, 90 \%)$ as an oil.

$\boldsymbol{R}_{\boldsymbol{f}}\left(20 \%\right.$ acetone-petrol) 0.21; IR (thin film, $\mathrm{cm}^{-1}$ ) 3434, 2979, 1735, 1703, 1455, 1395, 1221, 1172, 1116, 1039; ${ }^{1} \mathbf{H}$ NMR $\left(400 \mathrm{MHz}, \mathrm{CDCl}_{3}\right)$ ( 1.5:1 mixture of Boc rotamers) (major Boc rotamer) $\delta 7.27-7.35(\mathrm{~m}, 5 \mathrm{H}), 5.02(\mathrm{~d}, 1 \mathrm{H}, J=6.9 \mathrm{~Hz}), 4.90(\mathrm{~d}, 1 \mathrm{H}, J=6.9 \mathrm{~Hz}), 4.75(\mathrm{~d}, 1 \mathrm{H}$, $J=11.6 \mathrm{~Hz}), 4.64(\mathrm{q}, 1 \mathrm{H}, J=6.5 \mathrm{~Hz}), 4.60-4.51(\mathrm{~m}, 2 \mathrm{H}), 4.34-4.13(\mathrm{~m}, 3 \mathrm{H}), 4.00(\mathrm{br} \mathrm{s}, 1 \mathrm{H})$, $3.96(\mathrm{~d}, 1 \mathrm{H}, J=12.6 \mathrm{~Hz}), 3.40-3.33(\mathrm{~m}, 1 \mathrm{H}), 3.0($ br s, 1H), 1.38 (s, 9H), 1.29 (t, 3H, $J=7.1 \mathrm{~Hz}$ ) and $1.16(\mathrm{~d}, 3 \mathrm{H}, J=6.5 \mathrm{~Hz})$; (minor Boc rotamer) $\delta 7.27-7.35(\mathrm{~m}, 5 \mathrm{H}), 5.02(\mathrm{~d}$, $1 \mathrm{H}, J=6.9 \mathrm{~Hz}), 4.88(\mathrm{~d}, 1 \mathrm{H}, J=6.9 \mathrm{~Hz}), 4.82(\mathrm{q}, 1 \mathrm{H}, J=6.5 \mathrm{~Hz}), 4.73(\mathrm{~d}, 1 \mathrm{H}, J=11.9 \mathrm{~Hz})$, $4.60-4.51(\mathrm{~m}, 2 \mathrm{H}), 4.34-4.13(\mathrm{~m}, 3 \mathrm{H}), 4.00$ (br s, $1 \mathrm{H}), 3.82$ (d, $1 \mathrm{H}, J=12.6 \mathrm{~Hz}), 3.40-3.33$ (m, 1H), $3.0($ br s, 1H), $1.41(\mathrm{~s}, 9 \mathrm{H}), 1.27(\mathrm{t}, 3 \mathrm{H}, J=7.1 \mathrm{~Hz})$ and $1.13(\mathrm{~d}, 3 \mathrm{H}, J=6.5 \mathrm{~Hz})$; ${ }^{13}$ C NMR (100 MHz, $\mathrm{CDCl}_{3}$ ) (major Boc rotamer) $\delta 172.5$ (C), 152.9 (C), 137.6 (C), 128.5 $(2 \times \mathrm{CH}), 127.8(2 \times \mathrm{CH}), 127.8(\mathrm{CH}), 96.1\left(\mathrm{CH}_{2}\right), 81.2(\mathrm{C}), 77.6(\mathrm{CH}), 74.8(\mathrm{CH}), 72.0(\mathrm{C})$, $70.3(\mathrm{CH}), 70.0\left(\mathrm{CH}_{2}\right), 62.3\left(\mathrm{CH}_{2}\right), 54.1\left(\mathrm{CH}_{2}\right), 28.2\left(3 \times \mathrm{CH}_{3}\right), 16.2\left(\mathrm{CH}_{3}\right)$ and $14.0\left(\mathrm{CH}_{3}\right)$; (minor Boc rotamer) $\delta 172.7(\mathrm{C}), 154.0(\mathrm{C}), 137.8(\mathrm{C}), 128.4(2 \times \mathrm{CH}), 127.8(\mathrm{CH}), 127.6$ $(2 \times \mathrm{CH}), 96.1\left(\mathrm{CH}_{2}\right), 80.7(\mathrm{C}), 76.9(\mathrm{CH}), 73.9(\mathrm{CH}), 72.0(\mathrm{C}), 70.9(\mathrm{CH}), 70.0\left(\mathrm{CH}_{2}\right), 62.3$ $\left(\mathrm{CH}_{2}\right), 54.7\left(\mathrm{CH}_{2}\right), 28.2\left(3 \times \mathrm{CH}_{3}\right), 16.2\left(\mathrm{CH}_{3}\right)$ and $14.0\left(\mathrm{CH}_{3}\right)$; LRMS (ESI, $\left.m / z\right) 498(\mathrm{M}+$ $\left.\mathrm{NH}_{4}{ }^{+}+\mathrm{MeCN}, 100 \%\right)$; HRMS (ESI) calcd for $\mathrm{C}_{22} \mathrm{H}_{34} \mathrm{NO}_{8}\left(\mathrm{M}+\mathrm{H}^{+}\right)$440.2284; found $440.2287(\Delta 0.5 \mathrm{ppm})$. 
Iodohydrin 22

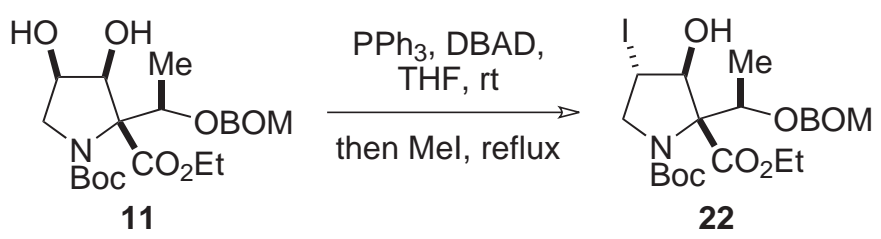

$\mathrm{PPh}_{3}(11.0 \mathrm{~g}, 42.1 \mathrm{mmol})$ and DBAD $(9.69 \mathrm{~g}, 42.1 \mathrm{mmol})$ were added to a solution of diol 11 $(6.16 \mathrm{~g}, 14.0 \mathrm{mmol})$ in THF $(120 \mathrm{~mL})$. The yellow solution was stirred at rt for $30 \mathrm{~min}$ and MeI $(6.1 \mathrm{~mL}, 98.0 \mathrm{mmol})$ was added dropwise. The resulting pale yellow suspension was heated at reflux for $15 \mathrm{~h}$. The reaction mixture was poured into $\mathrm{H}_{2} \mathrm{O}(80 \mathrm{~mL})$ and extracted with $\mathrm{CH}_{2} \mathrm{Cl}_{2}(3 \times 80 \mathrm{~mL})$. The organic extracts were dried with $\mathrm{MgSO}_{4}$, filtered and evaporated under reduced pressure. The residue was subjected to column chromatography (4\% $\mathrm{Me}_{2} \mathrm{CO}$-petrol) to give iodohydrin 22 (8.59 g) which was contaminated with DBAD residues but was used in the following step without further purification.

$\boldsymbol{R}_{\boldsymbol{f}}\left(20 \% \mathrm{Me}_{2} \mathrm{CO}\right.$-petrol) 0.42; IR (thin film, $\mathrm{cm}^{-1}$ ) 3346, 2979, 1745, 1704, 1455, 1389, 1251, 1164, 1027; ${ }^{1} \mathbf{H}$ NMR $\left(400 \mathrm{MHz}, \mathrm{CDCl}_{3}\right)(\sim 1.3: 1$ mixture of Boc rotamers) (major Boc rotamer) $\delta 7.36-7.22(\mathrm{~m}, 5 \mathrm{H}), 5.07(\mathrm{~d}, 1 \mathrm{H}, J=7.1 \mathrm{~Hz}), 4.86(\mathrm{~d}, 1 \mathrm{H}, J=7.1 \mathrm{~Hz}), 4.73(\mathrm{~d}, 1 \mathrm{H}$, $J=11.6 \mathrm{~Hz}), 4.59(\mathrm{q}, 1 \mathrm{H}, J=6.6 \mathrm{~Hz}), 4.59-4.50(\mathrm{~m}, 2 \mathrm{H}), 4.40-4.08(\mathrm{~m}, 4 \mathrm{H}), 3.52-3.38(\mathrm{~m}$, $1 \mathrm{H}), 3.01(\mathrm{~d}, 1 \mathrm{H}, J=2.5 \mathrm{~Hz}), 1.36(\mathrm{~s}, 9 \mathrm{H}), 1.26(\mathrm{t}, 3 \mathrm{H}, J=7.1 \mathrm{~Hz})$ and $1.18(\mathrm{~d}, 3 \mathrm{H}$, $J=6.6 \mathrm{~Hz})$; (minor Boc rotamer) $\delta 7.36-7.22(\mathrm{~m}, 5 \mathrm{H}), 5.07(\mathrm{~d}, 1 \mathrm{H}, J=7.1 \mathrm{~Hz}), 4.86(\mathrm{~d}, 1 \mathrm{H}$, $J=7.1 \mathrm{~Hz}), 4.76(\mathrm{q}, 1 \mathrm{H}, J=6.6 \mathrm{~Hz}), 4.73(\mathrm{~d}, 1 \mathrm{H}, J=11.6 \mathrm{~Hz}), 4.59-4.50$ (m, 2H), 4.40-4.08 $(\mathrm{m}, 4 \mathrm{H}), 3.52-3.38(\mathrm{~m}, 1 \mathrm{H}), 3.03(\mathrm{~d}, 1 \mathrm{H}, J=2.5 \mathrm{~Hz}), 1.41(\mathrm{~s}, 9 \mathrm{H}), 1.22(\mathrm{t}, 3 \mathrm{H}, J=7.6 \mathrm{~Hz})$ and $1.15(\mathrm{~d}, 3 \mathrm{H}, J=6.6 \mathrm{~Hz}) ;{ }^{13} \mathrm{C}$ NMR $\left(100 \mathrm{MHz}, \mathrm{CDCl}_{3}\right.$ ) (major Boc rotamer) $\delta 170.5(\mathrm{C})$, $152.1(\mathrm{C}), 137.5(\mathrm{C}), 128.5(2 \times \mathrm{CH}), 127.9(2 \times \mathrm{CH}), 127.9(\mathrm{CH}), 95.9\left(\mathrm{CH}_{2}\right), 82.0(\mathrm{CH})$, $81.5(\mathrm{C}), 77.9(\mathrm{CH}), 71.1(\mathrm{C}), 70.1\left(\mathrm{CH}_{2}\right), 61.6\left(\mathrm{CH}_{2}\right), 54.0\left(\mathrm{CH}_{2}\right), 28.2\left(3 \times \mathrm{CH}_{3}\right), 18.5(\mathrm{CH})$, $15.9\left(\mathrm{CH}_{3}\right)$ and $14.3\left(\mathrm{CH}_{3}\right)$; (minor Boc rotamer) $\delta 170.5(\mathrm{C}), 153.1(\mathrm{C}), 137.7(\mathrm{C}), 128.4(2 \times$ $\mathrm{CH}), 127.9(\mathrm{CH}), 127.7(2 \times \mathrm{CH}), 96.1\left(\mathrm{CH}_{2}\right), 81.1(\mathrm{CH}), 81.0(\mathrm{C}), 77.4(\mathrm{CH}), 70.9(\mathrm{C}), 69.9$ $\left(\mathrm{CH}_{2}\right), 61.4\left(\mathrm{CH}_{2}\right), 54.7\left(\mathrm{CH}_{2}\right), 28.1\left(3 \times \mathrm{CH}_{3}\right), 19.0(\mathrm{CH}), 16.1\left(\mathrm{CH}_{3}\right)$ and $14.3\left(\mathrm{CH}_{3}\right) ; \mathbf{L R M S}$ $($ ESI, $m / z) 608\left(\mathrm{M}+\mathrm{NH}_{4}{ }^{+}+\mathrm{MeCN}, 100 \%\right)$ and $572\left(\mathrm{M}+\mathrm{Na}^{+}, 20 \%\right)$; HRMS (ESI) calcd for $\mathrm{C}_{22} \mathrm{H}_{33} \mathrm{NO}_{7} \mathrm{I}\left(\mathrm{M}+\mathrm{H}^{+}\right)$550.1302; found 550.1302 ( $\left.\triangle 0.0 \mathrm{ppm}\right)$. 


\section{Hydroxyester 12}

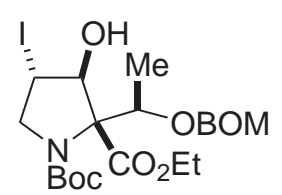

22

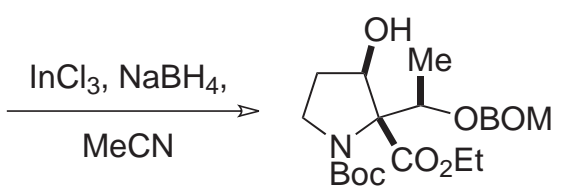

12

$\mathrm{InCl}_{3}(621 \mathrm{mg}, 2.81 \mathrm{mmol})$ was heated at $140{ }^{\circ} \mathrm{C}$ in vacuo for $1 \mathrm{~h}$. After the introduction of argon and addition of anhydrous $\mathrm{MeCN}(50 \mathrm{~mL})$, the solution was cooled down to $-78{ }^{\circ} \mathrm{C}$ and $\mathrm{NaBH}_{4}(850 \mathrm{mg}, 22.5 \mathrm{mmol})$ was added in one portion. The mixture was kept at this temperature for $10 \mathrm{~min}$. Upon warming to $\mathrm{rt}$, a solution of contaminated iodohydrin 22 $(8.91 \mathrm{~g})$ in anhydrous $\mathrm{MeCN}(40 \mathrm{~mL})$ was added via cannula and the reaction mixture was stirred at rt overnight. $\mathrm{H}_{2} \mathrm{O}(20 \mathrm{~mL})$ was added and the aqueous layer was extracted with $\mathrm{Et}_{2} \mathrm{O}$ $(3 \times 80 \mathrm{~mL})$. The combined organic extracts were washed with brine $(50 \mathrm{~mL})$, dried with $\mathrm{MgSO}_{4}$ and evaporated under reduced pressure. The crude product was purified by column chromatography $\left(20 \% \rightarrow 25 \% \mathrm{Et}_{2} \mathrm{O}\right.$-petrol) to give hydroxyester 12 (4.17 g, $9.85 \mathrm{mmol}, 70 \%$ over 2 steps) as an oil.

$\boldsymbol{R}_{f}\left(20 \% \mathrm{Me}_{2} \mathrm{CO}\right.$-petrol) 0.32; IR (thin film, $\mathrm{cm}^{-1}$ ) 3551, 2980, 1741, 1699, 1455, 1391, 1304, 1257, 1225, 1172, 1037, 859, 738, 699; ${ }^{1} \mathbf{H}$ NMR (400 MHz, $\left.\mathrm{CDCl}_{3}\right)(\sim 2: 1$ mixture of Boc rotamers) (major Boc rotamer) $\delta 7.39-7.24(\mathrm{~m}, 5 \mathrm{H}), 5.11(\mathrm{~d}, 1 \mathrm{H}, J=7.1 \mathrm{~Hz}), 4.90(\mathrm{~d}, 1 \mathrm{H}$, $J=7.1 \mathrm{~Hz}), 4.76(\mathrm{~d}, 1 \mathrm{H}, J=11.9 \mathrm{~Hz}), 4.69-4.57(\mathrm{~m}, 3 \mathrm{H}), 4.30-4.09(\mathrm{~m}, 2 \mathrm{H}), 3.88(\mathrm{t}, 1 \mathrm{H}$, $J=10.1 \mathrm{~Hz}$ ), 3.26-3.14 (m, 1H), 2.77 (br s, 1H), 2.30-2.04 (m, 2H), 1.39 (s, 9H), 1.28 (t, 3H, $J=7.1 \mathrm{~Hz})$ and $1.19(\mathrm{~d}, 3 \mathrm{H}, J=6.6 \mathrm{~Hz})$; (minor Boc rotamer) $\delta 7.39-7.24(\mathrm{~m}, 5 \mathrm{H}), 5.11(\mathrm{~d}$, $1 \mathrm{H}, J=7.1 \mathrm{~Hz}), 4.90(\mathrm{~d}, 1 \mathrm{H}, J=7.1 \mathrm{~Hz}), 4.83(\mathrm{q}, 1 \mathrm{H}, J=6.6 \mathrm{~Hz}), 4.76(\mathrm{~d}, 1 \mathrm{H}, J=11.9 \mathrm{~Hz})$, 4.67-4.57 (m, 2H), 4.30-4.09 (m, 2H), 3.75 (t, 1H, $J=10.1 \mathrm{~Hz}), 3.26-3.14(\mathrm{~m}, 1 \mathrm{H}), 2.83$ (br s, 1H), 2.30-2.04 (m, 2H), $1.42(\mathrm{~s}, 9 \mathrm{H}), 1.25(\mathrm{t}, 3 \mathrm{H}, J=7.1 \mathrm{~Hz})$ and $1.16(\mathrm{~d}, 3 \mathrm{H}, J=6.6 \mathrm{~Hz})$; ${ }^{13} \mathrm{C}$ NMR (100 MHz, $\mathrm{CDCl}_{3}$ ) (major Boc rotamer) $\delta 171.4(\mathrm{C}), 153.3(\mathrm{C}), 137.6(\mathrm{C}), 128.5$ $(2 \times \mathrm{CH}), 127.9(2 \times \mathrm{CH}), 127.7(\mathrm{CH}), 96.0\left(\mathrm{CH}_{2}\right), 80.7(\mathrm{C}), 78.7(\mathrm{CH}), 74.5(\mathrm{CH}), 71.5(\mathrm{C})$, $70.0\left(\mathrm{CH}_{2}\right), 61.1\left(\mathrm{CH}_{2}\right), 44.6\left(\mathrm{CH}_{2}\right), 29.1\left(\mathrm{CH}_{2}\right), 28.2\left(3 \times \mathrm{CH}_{3}\right), 15.9\left(\mathrm{CH}_{3}\right)$ and $14.3\left(\mathrm{CH}_{3}\right)$; (minor Boc rotamer) $\delta 171.3(\mathrm{C}), 154.2(\mathrm{C}), 137.9(\mathrm{C}), 128.4(2 \times \mathrm{CH}), 127.8(2 \times \mathrm{CH}), 127.6$ $(\mathrm{CH}), 96.2\left(\mathrm{CH}_{2}\right), 80.1(\mathrm{C}), 78.2(\mathrm{CH}), 73.6(\mathrm{CH}), 71.5(\mathrm{C}), 69.8\left(\mathrm{CH}_{2}\right), 61.0\left(\mathrm{CH}_{2}\right), 45.1$ $\left(\mathrm{CH}_{2}\right), 28.6\left(\mathrm{CH}_{2}\right), 28.3\left(3 \times \mathrm{CH}_{3}\right), 16.1\left(\mathrm{CH}_{3}\right)$ and $14.3\left(\mathrm{CH}_{3}\right)$; LRMS (ESI, $\left.\mathrm{m} / \mathrm{z}\right) 482$ $\left(\mathrm{M}+\mathrm{NH}_{4}{ }^{+}+\mathrm{MeCN}, 100 \%\right)$; HRMS (ESI) calcd for $\mathrm{C}_{22} \mathrm{H}_{34} \mathrm{NO}_{7}\left(\mathrm{M}+\mathrm{H}^{+}\right)$424.2335; found $424.2330(\Delta 1.3 \mathrm{ppm})$. 


\section{Ketoester 13}

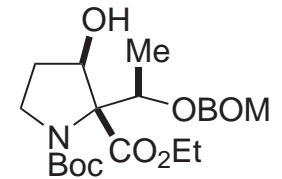

12

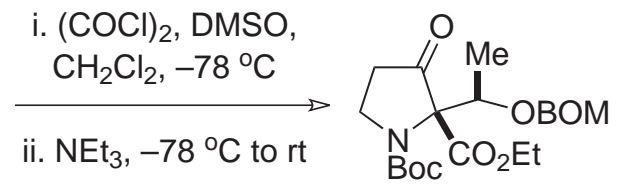

13

Oxalyl chloride $(1.4 \mathrm{~mL}, 15.8 \mathrm{mmol})$ and $\mathrm{CH}_{2} \mathrm{Cl}_{2}(30 \mathrm{~mL})$ were introduced to a flame-dried flask and cooled to $-78{ }^{\circ} \mathrm{C}$. A solution of anhydrous DMSO (2.2 mL, $\left.31.0 \mathrm{mmol}\right)$ in $\mathrm{CH}_{2} \mathrm{Cl}_{2}$ $(8 \mathrm{~mL})$ was added dropwise and the mixture was stirred at $-78{ }^{\circ} \mathrm{C}$ for $10 \mathrm{~min}$. A solution of hydroxyester $12(3.33 \mathrm{~g}, 7.87 \mathrm{mmol})$ in $\mathrm{CH}_{2} \mathrm{Cl}_{2}(30 \mathrm{~mL})$ was added dropwise and the mixture was stirred for $40 \mathrm{~min}$. Triethylamine $(5.5 \mathrm{~mL}, 39.5 \mathrm{mmol})$ was added dropwise and the reaction mixture was warmed to room to rt over $40 \mathrm{~min}$. The reaction mixture was poured into $\mathrm{H}_{2} \mathrm{O}(50 \mathrm{~mL})$ and the layers were separated. The organic extract was washed with aqueous $1 \mathrm{M} \mathrm{HCl}(50 \mathrm{~mL}), \mathrm{H}_{2} \mathrm{O}(3 \times 50 \mathrm{~mL})$ and brine $(50 \mathrm{~mL})$, dried with $\mathrm{MgSO}_{4}$, filtered and evaporated under reduced pressure. The residue was purified by flash column chromatography (10\% $\mathrm{Et}_{2} \mathrm{O}$-petrol) to give ketoester $\mathbf{1 3}$ (3.18 g, $\left.7.54 \mathrm{mmol}, 96 \%\right)$ as a pale yellow oil.

$\boldsymbol{R}_{f}\left(20 \% \mathrm{Me}_{2} \mathrm{CO}\right.$-petrol) 0.45; IR (thin film, $\mathrm{cm}^{-1}$ ) 2980, 1772, 1742, 1702, 1455, 1390, 1249 , 1162, 1025; ${ }^{1} \mathbf{H}$ NMR (400 MHz, $\left.\mathrm{C}_{6} \mathrm{D}_{6}\right)(\sim 1.2: 1$ mixture of Boc rotamers) (major Boc rotamer) $\delta 7.44-7.39(\mathrm{~m}, 2 \mathrm{H}), 7.29-7.15(\mathrm{~m}, 3 \mathrm{H}), 5.07(\mathrm{q}, 1 \mathrm{H}, J=6.3 \mathrm{~Hz}), 4.62(\mathrm{~d}, 1 \mathrm{H}$, $J=9.9 \mathrm{~Hz}), 4.57(\mathrm{~d}, 1 \mathrm{H}, J=9.9 \mathrm{~Hz}), 4.54(\mathrm{~d}, 1 \mathrm{H}, J=11.8 \mathrm{~Hz}), 4.46(\mathrm{~d}, 1 \mathrm{H}, J=11.8 \mathrm{~Hz})$, $4.32-3.81(\mathrm{~m}, 3 \mathrm{H}), 3.68-3.56(\mathrm{~m}, 1 \mathrm{H}), 2.49-2.34(\mathrm{~m}, 1 \mathrm{H}), 2.19$ (ddd, 1H, $J=18.6,8.3$ and $3.3 \mathrm{~Hz}), 1.56(\mathrm{~d}, 3 \mathrm{H}, J=6.3 \mathrm{~Hz}), 1.47(\mathrm{~s}, 9 \mathrm{H})$ and $0.99(\mathrm{t}, 3 \mathrm{H}, J=7.1 \mathrm{~Hz})$; (minor Boc rotamer) $\delta 7.44-7.39(\mathrm{~m}, 2 \mathrm{H}), 7.29-7.15(\mathrm{~m}, 3 \mathrm{H}), 5.18(\mathrm{q}, 1 \mathrm{H}, J=6.3 \mathrm{~Hz}), 4.59(\mathrm{~d}, 1 \mathrm{H}$, $J=9.8 \mathrm{~Hz}), 4.55(\mathrm{~d}, 1 \mathrm{H}, J=9.8 \mathrm{~Hz}), 4.54(\mathrm{~d}, 1 \mathrm{H}, J=11.8 \mathrm{~Hz}), 4.46(\mathrm{~d}, 1 \mathrm{H}, J=11.8 \mathrm{~Hz})$, $4.32-3.81(\mathrm{~m}, 3 \mathrm{H}), 3.68-3.56(\mathrm{~m}, 1 \mathrm{H}), 2.49-2.34$ (m, 1H), 2.29 (ddd, 1H, $J=18.4,8.7$ and $4.2 \mathrm{~Hz}), 1.73(\mathrm{~d}, 3 \mathrm{H}, J=6.3 \mathrm{~Hz}), 1.51(\mathrm{~s}, 9 \mathrm{H})$ and $1.05(\mathrm{t}, 3 \mathrm{H}, J=7.1 \mathrm{~Hz}) ;{ }^{13} \mathbf{C ~ N M R}$ $\left(100 \mathrm{MHz}, \mathrm{C}_{6} \mathrm{D}_{6}\right.$ ) (major Boc rotamer) $\delta 208.5$ (C), 166.6 (C), 153.7 (C), 138.3 (C), 128.6 $(2 \times \mathrm{CH}), 128.1(2 \times \mathrm{CH}), 127.8(\mathrm{CH}), 92.9\left(\mathrm{CH}_{2}\right), 80.8(\mathrm{C}), 77.7(\mathrm{CH}), 75.4(\mathrm{C}), 69.9\left(\mathrm{CH}_{2}\right)$, $61.5\left(\mathrm{CH}_{2}\right), 43.1\left(\mathrm{CH}_{2}\right), 36.0\left(\mathrm{CH}_{2}\right), 28.1\left(3 \times \mathrm{CH}_{3}\right), 16.3\left(\mathrm{CH}_{3}\right)$ and $13.8\left(\mathrm{CH}_{3}\right) ;$ (minor Boc rotamer) $\delta 207.5(\mathrm{C}), 166.2(\mathrm{C}), 154.4(\mathrm{C}), 138.3(\mathrm{C}), 128.5(2 \times \mathrm{CH}), 128.3(2 \times \mathrm{CH}), 127.5$ $(\mathrm{CH}), 92.9\left(\mathrm{CH}_{2}\right), 80.1(\mathrm{C}), 78.1(\mathrm{CH}), 75.5(\mathrm{C}), 69.9\left(\mathrm{CH}_{2}\right), 61.5\left(\mathrm{CH}_{2}\right), 43.4\left(\mathrm{CH}_{2}\right), 36.8$ $\left(\mathrm{CH}_{2}\right), 28.2\left(3 \times \mathrm{CH}_{3}\right), 16.4\left(\mathrm{CH}_{3}\right)$ and $14.0\left(\mathrm{CH}_{3}\right)$; LRMS (ESI, $\left.m / z\right) 480\left(\mathrm{M}+\mathrm{NH}_{4}{ }^{+}+\right.$ MeCN, 100\%); HRMS (ESI) calcd for $\mathrm{C}_{22} \mathrm{H}_{31} \mathrm{NO}_{7} \mathrm{Na}\left(\mathrm{M}+\mathrm{Na}^{+}\right) 444.1998$; found 444.1996 $(\triangle 0.6 \mathrm{ppm})$. 


\section{Methylated ketoester 14}

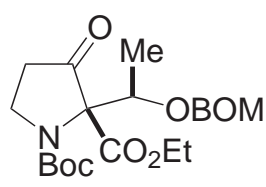

13
1) NaHMDS, 15-crown-5, $\mathrm{THF},-78^{\circ} \mathrm{C}$ then $\mathrm{Mel}$

2) NaHMDS, 15-crown-5, $-78^{\circ} \mathrm{C}$ then 2,6-di-tert-butylphenol

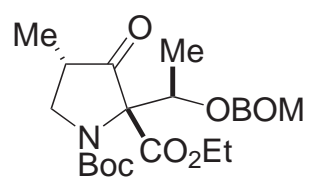

14

NaHMDS (1M in THF) (2.4 mL, $2.40 \mathrm{mmol})$ was added to a stirred solution of ketoester $\mathbf{1 3}$ (963 mg, $2.29 \mathrm{mmol})$ and 15 -crown-5 $(0.48 \mathrm{~mL}, 2.40 \mathrm{mmol})$ in THF $(20 \mathrm{~mL})$ at $-78^{\circ} \mathrm{C}$. After $30 \mathrm{~min}$ MeI $(0.86 \mathrm{~mL}, 13.8 \mathrm{mmol})$ was added dropwise. The reaction mixture was stirred at $-78{ }^{\circ} \mathrm{C}$ for $5 \mathrm{~h}$ and quenched with saturated $\mathrm{NH}_{4} \mathrm{Cl}(5 \mathrm{~mL})$. The layers were separated and the aqueous layer was extracted with $\mathrm{Et}_{2} \mathrm{O}(3 \times 25 \mathrm{~mL})$. The combined organic extracts were dried with $\mathrm{MgSO}_{4}$, filtered and evaporated under reduced pressure. The crude product was purified by gradient flash column chromatography $\left(12 \% \rightarrow 16 \% \mathrm{Et}_{2} \mathrm{O}\right.$-petrol) to first yield the dimethylated ketone (61.2 mg, $0.136 \mathrm{mmol}, 6 \%$ ), followed by the methylated ketoester (754 $\mathrm{mg}, 1.73 \mathrm{mmol}, 76 \%)$ as an inseparable mixture of diastereomers, and finally recovered ketoester 13 (51.7 mg, $0.123 \mathrm{mmol}, 5 \%)$. NaHMDS (1M in THF) (2.6 mL, $2.60 \mathrm{mmol}$ ) was added to a stirred solution of the methylated ketoester (932 mg, $2.14 \mathrm{mmol}$ ) and 15-crown-5 $(0.51 \mathrm{~mL}, 2.60 \mathrm{mmol})$ in THF $(20 \mathrm{~mL})$ at $-78{ }^{\circ} \mathrm{C}$. After $30 \mathrm{~min}$ a solution of 2,6-di-tertbutylphenol $(1.33 \mathrm{~g}, 6.42 \mathrm{mmol})$ in THF $(10 \mathrm{~mL})$ was added dropwise. The reaction mixture was stirred at $-78{ }^{\circ} \mathrm{C}$ for $5 \mathrm{~h}$ and quenched with saturated $\mathrm{NH}_{4} \mathrm{Cl}(5 \mathrm{~mL})$. The layers were separated and the aqueous layer was extracted with $\mathrm{Et}_{2} \mathrm{O}(3 \times 25 \mathrm{~mL})$. The combined organic extracts were dried with $\mathrm{MgSO}_{4}$, filtered and evaporated under reduced pressure. The crude product was purified by column chromatography (15\% $\mathrm{Et}_{2} \mathrm{O}$-petrol) to yield the methylated ketoester 14 (894 mg, $2.05 \mathrm{mmol}, 96 \%)$ as a pale yellow oil and as almost exclusively one diastereomer $(\mathrm{dr}>10: 1)$.

$\boldsymbol{R}_{f}$ (40\% $\mathrm{Et}_{2} \mathrm{O}$-petrol) 0.44; IR (thin film, $\mathrm{cm}^{-1}$ ) 2979, 1770, 1742, 1703, 1455, 1392, 1244, 1162, 1038; ${ }^{1} \mathbf{H}$ NMR $\left(400 \mathrm{MHz}, \mathrm{C}_{6} \mathrm{D}_{6}\right.$ ) (major Boc rotamer) $\delta$ 7.32-7.27 (m, 2H), 7.15-7.03 $(\mathrm{m}, 3 \mathrm{H}), 5.00(\mathrm{q}, 1 \mathrm{H}, J=6.3 \mathrm{~Hz}), 4.58(\mathrm{t}, 1 \mathrm{H}, J=10.3 \mathrm{~Hz}) ; 4.53-4.32(\mathrm{~m}, 4 \mathrm{H}), 3.98(\mathrm{dq}, 1 \mathrm{H}$, $J=10.8$ and $7.1 \mathrm{~Hz}), 3.75(\mathrm{dq}, 1 \mathrm{H}, J=10.8$ and $7.1 \mathrm{~Hz}), 3.20(\mathrm{t}, 1 \mathrm{H}, J=10.3 \mathrm{~Hz}), 2.46(\mathrm{dq}$, $1 \mathrm{H}, J=10.0$ and $7.0 \mathrm{~Hz}), 1.48(\mathrm{~d}, 3 \mathrm{H}, J=6.3 \mathrm{~Hz}), 1.37(\mathrm{~s}, 9 \mathrm{H}), 0.88(\mathrm{~d}, 3 \mathrm{H}, J=6.9 \mathrm{~Hz})$ and $0.87(\mathrm{t}, 3 \mathrm{H}, J=7.2 \mathrm{~Hz}) ;$ (minor Boc rotamer) $\delta 7.32-7.27(\mathrm{~m}, 2 \mathrm{H}), 7.15-7.03(\mathrm{~m}, 3 \mathrm{H}), 5.11$ $(\mathrm{q}, 1 \mathrm{H}, J=6.3 \mathrm{~Hz}), 4.53-4.32(\mathrm{~m}, 4 \mathrm{H}), 4.18(\mathrm{t}, 1 \mathrm{H}, J=10.1 \mathrm{~Hz}), 4.12(\mathrm{dq}, 1 \mathrm{H}, J=10.8$ and $7.1 \mathrm{~Hz}), 3.90(\mathrm{dq}, 1 \mathrm{H}, J=10.8$ and $7.1 \mathrm{~Hz}), 3.21(\mathrm{t}, 1 \mathrm{H}, J=10.1 \mathrm{~Hz}), 2.52(\mathrm{dq}, 1 \mathrm{H}$, $J=9.8$ and $6.9 \mathrm{~Hz}), 1.64(\mathrm{~d}, 3 \mathrm{H}, J=6.3 \mathrm{~Hz}), 1.42(\mathrm{~s}, 9 \mathrm{H}), 0.94(\mathrm{t}, 3 \mathrm{H}, J=7.0 \mathrm{~Hz})$ and $0.93(\mathrm{~d}$, $3 \mathrm{H}, J=6.8 \mathrm{~Hz}) ;{ }^{13} \mathrm{C}$ NMR $\left(100 \mathrm{MHz}, \mathrm{C}_{6} \mathrm{D}_{6}\right)$ (major Boc rotamer) $\delta 210.9(\mathrm{C}), 166.4(\mathrm{C})$, 
$153.4(\mathrm{C}), 138.3(\mathrm{C}), 128.6(2 \times \mathrm{CH}), 128.1(2 \times \mathrm{CH}), 127.8(\mathrm{CH}), 92.9\left(\mathrm{CH}_{2}\right), 80.7(\mathrm{C}), 78.0$ $(\mathrm{CH}), 75.9(\mathrm{C}), 69.9\left(\mathrm{CH}_{2}\right), 61.5\left(\mathrm{CH}_{2}\right), 50.4\left(\mathrm{CH}_{2}\right), 41.6(\mathrm{CH}), 28.1\left(3 \times \mathrm{CH}_{3}\right), 16.1\left(\mathrm{CH}_{3}\right)$, $13.8\left(\mathrm{CH}_{3}\right)$ and $11.6\left(\mathrm{CH}_{3}\right)$; (minor Boc rotamer) $\delta 210.1(\mathrm{C}), 166.0(\mathrm{C}), 153.9(\mathrm{C}), 138.4(\mathrm{C})$, $128.5(2 \times \mathrm{CH}), 128.3(2 \times \mathrm{CH}), 127.7(\mathrm{CH}), 92.9\left(\mathrm{CH}_{2}\right), 80.0(\mathrm{C}), 78.5(\mathrm{CH}), 75.9(\mathrm{C}), 69.9$ $\left(\mathrm{CH}_{2}\right), 61.5\left(\mathrm{CH}_{2}\right), 51.0\left(\mathrm{CH}_{2}\right), 42.2(\mathrm{CH}), 28.2\left(3 \times \mathrm{CH}_{3}\right), 16.2\left(\mathrm{CH}_{3}\right), 14.0\left(\mathrm{CH}_{3}\right)$ and 11.7 $\left(\mathrm{CH}_{3}\right)$. LRMS (ESI, $\left.m / z\right) 458\left(\mathrm{M}+\mathrm{Na}^{+}, 100 \%\right)$ and $494\left(\mathrm{M}+\mathrm{NH}_{4}{ }^{+}+\mathrm{MeCN}, 60 \%\right)$; HRMS (ESI) calcd for $\mathrm{C}_{23} \mathrm{H}_{33} \mathrm{NO}_{7} \mathrm{Na}\left(\mathrm{M}+\mathrm{Na}^{+}\right)$458.2155; found $458.2165(\Delta 2.3 \mathrm{ppm})$.

\section{Homoallyl alcohol 15}

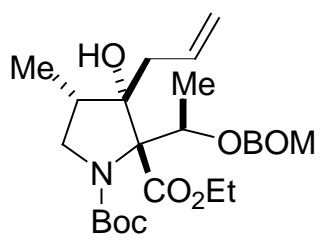

Allylmagnesium bromide (1.0 $\mathrm{M}$ in $\left.\mathrm{Et}_{2} \mathrm{O}\right)(5.9 \mathrm{~mL}, 5.90 \mathrm{mmol})$ was added dropwise to a stirred solution of methylketone $14(357 \mathrm{mg}, 0.821 \mathrm{mmol})$ in $\mathrm{Et}_{2} \mathrm{O}(50 \mathrm{~mL})$ at $-78^{\circ} \mathrm{C}$. The reaction mixture was stirred for $3 \mathrm{~h}$, quenched with saturated $\mathrm{NH}_{4} \mathrm{Cl}(5 \mathrm{~mL})$, poured into water and the layers were separated. The aqueous phase was extracted with $\mathrm{Et}_{2} \mathrm{O}$ $(3 \times 30 \mathrm{~mL})$ and the combined organic extracts were washed with brine, dried with $\mathrm{MgSO}_{4}$ and evaporated under reduced pressure. The crude product was purified by flash column chromatography (10.5\% Et $2 \mathrm{O}$-petrol) to give homoallyl alcohol 15 (338 mg, $0.709 \mathrm{mmol}$, $86 \%)$ as an oil.

$\boldsymbol{R}_{f}$ (40\% $\mathrm{Et}_{2} \mathrm{O}$-petrol) 0.65; IR (thin film, $\mathrm{cm}^{-1}$ ) 3514, 2979, 1737, 1695, 1455, 1402. 1257, 1162, 1027; ${ }^{1} \mathbf{H}$ NMR $\left(400 \mathrm{MHz}, \mathrm{CDCl}_{3}\right)$ ( 1.3:1 mixture of Boc rotamers) (major Boc rotamer) $\delta 7.39-7.27(\mathrm{~m}, 5 \mathrm{H}), 5.87(\mathrm{dddd}, 1 \mathrm{H}, J=10.5,8.2,6.0$ and $2.1 \mathrm{~Hz}), 5.15(\mathrm{~d}, 1 \mathrm{H}$, $J=7.1 \mathrm{~Hz}), 5.06-4.96(\mathrm{~m}, 2 \mathrm{H}), 4.91(\mathrm{~d}, 1 \mathrm{H}, J=7.1 \mathrm{~Hz}), 4.76(\mathrm{~d}, 1 \mathrm{H}, J=11.6 \mathrm{~Hz}), 4.74(\mathrm{q}$, $1 \mathrm{H}, J=6.8 \mathrm{~Hz}), 4.59(\mathrm{~d}, 1 \mathrm{H}, J=11.6 \mathrm{~Hz}), 4.26-4.04(\mathrm{~m}, 2 \mathrm{H}), 3.96(\mathrm{dd}, 1 \mathrm{H}, J=10.1 \mathrm{and}$ $7.8 \mathrm{~Hz}$ ), 3.62 (br s, 1H), 3.18 (dd, 1H, $J=11.6$ and $10.3 \mathrm{~Hz}$ ), 2.68-2.58 (m, 1H), 2.43-2.22 $(\mathrm{m}, 2 \mathrm{H}), 1.40(\mathrm{~d}, 3 \mathrm{H}, J=6.8 \mathrm{~Hz}), 1.39(\mathrm{~s}, 9 \mathrm{H}), 1.30(\mathrm{t}, 3 \mathrm{H}, J=7.1 \mathrm{~Hz})$, and $1.02(\mathrm{~d}, 3 \mathrm{H}$, $J=6.6 \mathrm{~Hz}$ ); (minor Boc rotamer) 7.39-7.27 (m, 5H), 5.93 (dddd, 1H, $J=10.4,8.4,6.0$ and $2.1 \mathrm{~Hz}), 5.15(\mathrm{~d}, 1 \mathrm{H}, J=7.1 \mathrm{~Hz}), 5.06-4.96(\mathrm{~m}, 2 \mathrm{H}), 4.94(\mathrm{q}, 1 \mathrm{H}, J=6.8 \mathrm{~Hz}), 4.91(\mathrm{~d}, 1 \mathrm{H}$, $J=7.1 \mathrm{~Hz}), 4.76(\mathrm{~d}, 1 \mathrm{H}, J=11.6 \mathrm{~Hz}), 4.57(\mathrm{~d}, 1 \mathrm{H}, J=11.6 \mathrm{~Hz}), 4.26-4.04(\mathrm{~m}, 2 \mathrm{H}), 3.84(\mathrm{dd}$, $1 \mathrm{H}, J=10.1$ and $7.8 \mathrm{~Hz}$ ), $3.63(\mathrm{br} \mathrm{s}, 1 \mathrm{H}), 3.17(\mathrm{dd}, 1 \mathrm{H}, J=11.6$ and $10.3 \mathrm{~Hz}), 2.68-2.58(\mathrm{~m}$, 1H), 2.43-2.22 (m, 2H), $1.44(\mathrm{~s}, 9 \mathrm{H}), 1.36(\mathrm{~d}, 3 \mathrm{H}, J=6.8 \mathrm{~Hz}), 1.26(\mathrm{t}, 3 \mathrm{H}, J=7.1 \mathrm{~Hz})$ and $1.01(\mathrm{~d}, 3 \mathrm{H}, J=6.6 \mathrm{~Hz}) ;{ }^{13} \mathbf{C} \mathbf{~ N M R}\left(100 \mathrm{MHz}, \mathrm{CDCl}_{3}\right.$ ) (major Boc rotamer) $\delta 172.8(\mathrm{C})$, 
$152.6(\mathrm{C}), 137.5(\mathrm{C}), 133.7(\mathrm{CH}), 128.5(2 \times \mathrm{CH}), 128.0(2 \times \mathrm{CH}), 127.9(\mathrm{CH}), 117.2\left(\mathrm{CH}_{2}\right)$, $96.0\left(\mathrm{CH}_{2}\right), 86.6(\mathrm{C}), 80.9(\mathrm{CH}), 80.6(\mathrm{C}), 76.6(\mathrm{C}), 70.1\left(\mathrm{CH}_{2}\right), 61.3\left(\mathrm{CH}_{2}\right), 53.0\left(\mathrm{CH}_{2}\right), 43.3$ $\left(\mathrm{CH}_{2}\right), 39.9(\mathrm{CH}), 28.3\left(3 \times \mathrm{CH}_{3}\right), 18.6\left(\mathrm{CH}_{3}\right), 14.0\left(\mathrm{CH}_{3}\right)$ and $10.0\left(\mathrm{CH}_{3}\right)$; (minor Boc rotamer) $\delta 172.7(\mathrm{C}), 153.9(\mathrm{C}), 137.7(\mathrm{C}), 133.9(\mathrm{CH}), 128.4(2 \times \mathrm{CH}), 127.9(2 \times \mathrm{CH})$, 127.6 (CH), $117.1\left(\mathrm{CH}_{2}\right), 96.2\left(\mathrm{CH}_{2}\right), 85.3(\mathrm{C}), 80.4(\mathrm{CH}), 79.9(\mathrm{C}), 76.6(\mathrm{C}), 69.9\left(\mathrm{CH}_{2}\right)$, $61.0\left(\mathrm{CH}_{2}\right), 53.4\left(\mathrm{CH}_{2}\right), 43.2\left(\mathrm{CH}_{2}\right), 40.4(\mathrm{CH}), 28.4\left(3 \times \mathrm{CH}_{3}\right), 18.7\left(\mathrm{CH}_{3}\right), 14.1\left(\mathrm{CH}_{3}\right)$ and $10.0\left(\mathrm{CH}_{3}\right)$; LRMS (ESI, $\left.m / z\right) 536\left(\mathrm{M}+\mathrm{NH}_{4}{ }^{+}+\mathrm{MeCN}, 100 \%\right)$ and $500\left(\mathrm{M}+\mathrm{Na}^{+}, 45 \%\right)$; HRMS (ESI) calcd for $\mathrm{C}_{26} \mathrm{H}_{39} \mathrm{NNaO}_{7}\left(\mathrm{M}+\mathrm{Na}^{+}\right)$500.2625; found $500.2619(\Delta 1.0 \mathrm{ppm})$.

\section{Allyl alcohol 16}

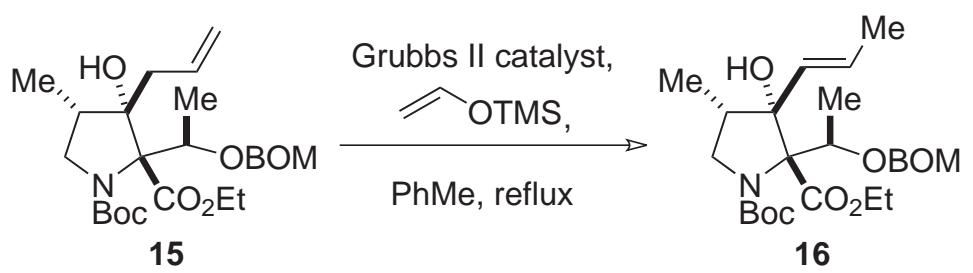

Vinyloxytrimethylsilane $(1.2 \mathrm{~mL}, 8.24 \mathrm{mmol})$ was added dropwise to a solution of homoallyl alcohol 15 (393 mg, $0.824 \mathrm{mmol})$ in anhydrous PhMe (40 mL). Grubbs II catalyst $(35.0 \mathrm{mg}$, $41.2 \mu \mathrm{mol})$ was added in one portion. The reaction mixture was heated to vigorous reflux for 15 h. Upon cooling, the solvent was evaporated under reduced pressure and the crude alkene (401 mg) could be used in the next step without further purification. However, a sample for characterisation was obtained by flash column chromatography (15\% Et $\mathrm{E}_{2} \mathrm{O}$-petrol), which gave allyl alcohol $\mathbf{1 6}$ as a pale yellow oil.

$\boldsymbol{R}_{\boldsymbol{f}}\left(20 \% \mathrm{Et}_{2} \mathrm{O}\right.$-petrol) 0.46; IR (thin film, $\mathrm{cm}^{-1}$ ) 3507, 2979, 1737, 1694, 1454, 1400, 1255, 1230, 1170, 1125, 1037; ${ }^{1} \mathbf{H}$ NMR (400 MHz, $\left.\mathrm{C}_{6} \mathrm{D}_{6}\right)$ ( 1.4:1 mixture of Boc rotamers) (major Boc rotamer) $\delta 7.40(\mathrm{~d}, 2 \mathrm{H}, J=7.3 \mathrm{~Hz}), 7.28-7.15(\mathrm{~m}, 3 \mathrm{H}), 5.78(\mathrm{dq}, 1 \mathrm{H}, J=15.4$ and $6.3 \mathrm{~Hz}), 5.67(\mathrm{dd}, 1 \mathrm{H}, J=15.4$ and $1.1 \mathrm{~Hz}), 5.46(\mathrm{q}, 1 \mathrm{H}, J=6.7 \mathrm{~Hz}), 5.31(\mathrm{~d}, 1 \mathrm{H}, J=6.4 \mathrm{~Hz})$, $4.83(\mathrm{~d}, 1 \mathrm{H}, J=6.4 \mathrm{~Hz}), 4.72(\mathrm{~d}, 1 \mathrm{H}, J=12.1 \mathrm{~Hz}), 4.53(\mathrm{~d}, 1 \mathrm{H}, J=12.1 \mathrm{~Hz}), 4.26-3.91(\mathrm{~m}$, $2 \mathrm{H}), 4.16(\mathrm{dd}, 1 \mathrm{H}, J=9.9$ and $7.9 \mathrm{~Hz}), 4.10(\mathrm{~d}, 1 \mathrm{H}, J=1.7 \mathrm{~Hz}), 3.58(\mathrm{dd}, 1 \mathrm{H}, J=11.3$ and $9.9 \mathrm{~Hz}), 2.80-2.63(\mathrm{~m}, 1 \mathrm{H}), 1.71(\mathrm{~d}, 3 \mathrm{H}, J=6.7 \mathrm{~Hz}), 1.65(\mathrm{td}, 3 \mathrm{H}, J=6.3$ and $1.1 \mathrm{~Hz}), 1.55$ (s, 9H), $1.13(\mathrm{~d}, 3 \mathrm{H}, J=6.8 \mathrm{~Hz})$ and $1.07(\mathrm{t}, 3 \mathrm{H}, J=7.2 \mathrm{~Hz})$; (minor Boc rotamer) $\delta 7.40(\mathrm{~d}$, $2 \mathrm{H}, J=7.3 \mathrm{~Hz}), 7.28-7.15(\mathrm{~m}, 3 \mathrm{H}), 5.72(\mathrm{dq}, 1 \mathrm{H}, J=15.5$ and $6.3 \mathrm{~Hz}), 5.61(\mathrm{dd}, 1 \mathrm{H}$, $J=15.5$ and $1.1 \mathrm{~Hz}), 5.36(\mathrm{~d}, 1 \mathrm{H}, J=6.7 \mathrm{~Hz}), 5.12(\mathrm{q}, 1 \mathrm{H}, J=6.8 \mathrm{~Hz}), 4.80(\mathrm{~d}, 1 \mathrm{H}$, $J=6.7 \mathrm{~Hz}), 4.72(\mathrm{~d}, 1 \mathrm{H}, J=12.1 \mathrm{~Hz}), 4.53(\mathrm{~d}, 1 \mathrm{H}, J=12.1 \mathrm{~Hz}), 4.50(\mathrm{dd}, 1 \mathrm{H}, J=10.1$ and $7.7 \mathrm{~Hz}), 4.26-3.91(\mathrm{~m}, 2 \mathrm{H}), 4.06(\mathrm{~d}, 1 \mathrm{H}, J=1.7 \mathrm{~Hz}), 3.62(\mathrm{dd}, 1 \mathrm{H}, J=11.3$ and $10.1 \mathrm{~Hz})$, 2.80-2.63 (m, 1H), $1.69(\mathrm{~d}, 3 \mathrm{H}, J=6.8 \mathrm{~Hz}), 1.65(\mathrm{td}, 1 \mathrm{H}, J=6.3$ and $1.1 \mathrm{~Hz}), 1.46(\mathrm{~s}, 9 \mathrm{H})$, 
$1.09(\mathrm{~d}, 3 \mathrm{H}, J=6.5 \mathrm{~Hz})$ and $1.05(\mathrm{t}, 3 \mathrm{H}, J=7.2 \mathrm{~Hz}) ;{ }^{13} \mathrm{C} \mathbf{N M R}\left(100 \mathrm{MHz}, \mathrm{C}_{6} \mathrm{D}_{6}\right)($ major Boc rotamer) $\delta 172.4(\mathrm{C}), 152.7(\mathrm{C}), 137.6(\mathrm{C}), 132.3(\mathrm{CH}), 128.4(2 \times \mathrm{CH}), 127.8(2 \times \mathrm{CH})$, $127.7(\mathrm{CH}), 125.2(\mathrm{CH}), 95.8\left(\mathrm{CH}_{2}\right), 87.8(\mathrm{C}), 80.4(\mathrm{C}), 80.2(\mathrm{CH}), 77.4(\mathrm{C}), 69.9\left(\mathrm{CH}_{2}\right), 61.2$ $\left(\mathrm{CH}_{2}\right), 52.7\left(\mathrm{CH}_{2}\right), 38.1(\mathrm{CH}), 28.2\left(3 \times \mathrm{CH}_{3}\right), 18.8\left(\mathrm{CH}_{3}\right), 17.9\left(\mathrm{CH}_{3}\right), 14.1\left(\mathrm{CH}_{3}\right)$ and 8.7 $\left(\mathrm{CH}_{3}\right)$; (minor Boc rotamer) $\delta 172.4(\mathrm{C}), 154.1(\mathrm{C}), 137.9(\mathrm{C}), 132.5(\mathrm{CH}), 128.3(2 \times \mathrm{CH})$, $127.6(2 \times \mathrm{CH}), 127.5(\mathrm{CH}), 125.1(\mathrm{CH}), 95.9\left(\mathrm{CH}_{2}\right), 86.5(\mathrm{C}), 79.8(\mathrm{C}), 79.6(\mathrm{CH}), 77.4(\mathrm{C})$, 69.8 $\left(\mathrm{CH}_{2}\right), 61.0\left(\mathrm{CH}_{2}\right), 53.1\left(\mathrm{CH}_{2} \mathrm{~N}\right), 38.8(\mathrm{CH}), 28.3\left(3 \times \mathrm{CH}_{3}\right), 18.8\left(\mathrm{CH}_{3}\right), 17.8\left(\mathrm{CH}_{3}\right), 14.1$ $\left(\mathrm{CH}_{3}\right)$ and $8.7\left(\mathrm{CH}_{3}\right)$; LRMS (ESI, $\left.\mathrm{m} / z\right) 536\left(\mathrm{M}+\mathrm{NH}_{4}{ }^{+}+\mathrm{MeCN}, 100 \%\right)$; HRMS (ESI) calcd for $\mathrm{C}_{26} \mathrm{H}_{39} \mathrm{NNaO}_{7}\left(\mathrm{M}+\mathrm{Na}^{+}\right)$500.2625; found 500.2617 ( $\left.\Delta 1.4 \mathrm{ppm}\right)$.

\section{1,2-Diol 23}
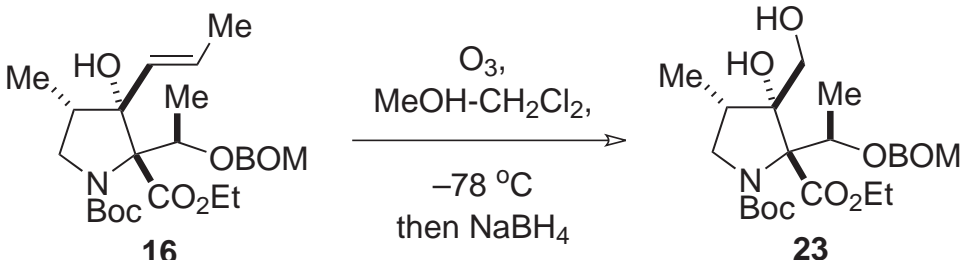

Ozone was bubbled through a solution of crude allyl alcohol $16(401 \mathrm{mg})$ in a 1:1 $\mathrm{CH}_{2} \mathrm{Cl}_{2^{-}}$ $\mathrm{MeOH}$ mixture $(10 \mathrm{~mL})$ at $-78{ }^{\circ} \mathrm{C}$ for $15 \mathrm{~min}$. $\mathrm{NaBH}_{4}(93.1 \mathrm{mg}, 2.46 \mathrm{mmol})$ was added and the reaction mixture was stirred at $-78{ }^{\circ} \mathrm{C}$ for $3 \mathrm{~h} . \mathrm{H}_{2} \mathrm{O}(1 \mathrm{~mL})$ was added and the organic solvents were evaporated under reduced pressure. The remaining aqueous residue was extracted with $\mathrm{CH}_{2} \mathrm{Cl}_{2}(3 \times 20 \mathrm{~mL})$, dried with $\mathrm{MgSO}_{4}$, filtered and evaporated under reduced pressure to give 1,2-diol $\mathbf{2 3}(390 \mathrm{mg}$ ) which could be used directly in the next step without further purification. However, a sample for characterisation was obtained by flash column chromatography (30\% $\mathrm{Et}_{2} \mathrm{O}$-petrol) which gave 1,2-diol 23 as an oil.

$\boldsymbol{R}_{\boldsymbol{f}}\left(60 \% \mathrm{Et}_{2} \mathrm{O}\right.$-petrol) 0.22; IR (thin film, $\mathrm{cm}^{-1}$ ) 3494, 2978, 1734, 1694, 1455, 1396, 1257, 1165, 1026; ${ }^{1} \mathbf{H}$ NMR $\left(400 \mathrm{MHz}, \mathrm{CDCl}_{3}\right)(\sim 1: 1$ mixture of Boc rotamers) $\delta 7.38-7.27$ (m, $10 \mathrm{H}), 5.09(\mathrm{~d}, 1 \mathrm{H}, J=6.8 \mathrm{~Hz}), 5.08(\mathrm{~d}, 1 \mathrm{H}, J=6.8 \mathrm{~Hz}), 4.98(\mathrm{~d}, 1 \mathrm{H}, J=6.8 \mathrm{~Hz}), 4.98(\mathrm{~d}, 1 \mathrm{H}$, $J=6.8 \mathrm{~Hz}), 4.86(\mathrm{q}, 1 \mathrm{H}, J=6.8 \mathrm{~Hz}), 4.76(\mathrm{~d}, 1 \mathrm{H}, J 11.6 \mathrm{~Hz}), 4.75(\mathrm{~d}, 1 \mathrm{H}, J=11.6 \mathrm{~Hz}), 4.69$ $(\mathrm{q}, 1 \mathrm{H}, J=6.8 \mathrm{~Hz}), 4.65(\mathrm{~d}, 1 \mathrm{H}, J=11.6 \mathrm{~Hz}), 4.63(\mathrm{~d}, 1 \mathrm{H}, J=11.6 \mathrm{~Hz}), 4.29-4.08(\mathrm{~m}, 4 \mathrm{H})$, $4.02(\mathrm{dd}, 1 \mathrm{H}, J=10.2$ and $7.9 \mathrm{~Hz}), 3.89(\mathrm{dd}, 1 \mathrm{H}, J 10.2$ and $7.9 \mathrm{~Hz}), 3.64(\mathrm{~d}, 1 \mathrm{H}, J=1.7 \mathrm{~Hz})$, $3.59(\mathrm{~d}, 1 \mathrm{H}, J=1.7 \mathrm{~Hz}), 3.48(\mathrm{~s}, 4 \mathrm{H}), 3.21(\mathrm{dd}, 1 \mathrm{H}, J 11.6$ and $10.2 \mathrm{~Hz}), 3.20(\mathrm{dd}, 1 \mathrm{H}$, $J=11.6$ and $10.2 \mathrm{~Hz}$ ), 3.12 (br s, 1H), 2.94 (br s, 1H), 2.35-2.22 (m, 2H), 1.44 (s, 9H), 1.43 $(\mathrm{d}, 3 \mathrm{H}, J=6.8 \mathrm{~Hz}), 1.39(\mathrm{~s}, 9 \mathrm{H}), 1.39(\mathrm{~d}, 3 \mathrm{H}, J=6.8 \mathrm{~Hz}), 1.30(\mathrm{t}, 3 \mathrm{H}, J=7.2 \mathrm{~Hz}), 1.26(\mathrm{t}, 3 \mathrm{H}$, $J=7.2 \mathrm{~Hz}), 1.01(\mathrm{~d}, 3 \mathrm{H}, J=6.5 \mathrm{~Hz})$ and $0.99(\mathrm{~d}, 3 \mathrm{H}, J=6.5 \mathrm{~Hz}) ;{ }^{13} \mathbf{C} \mathbf{N M R}(100 \mathrm{MHz}$, $\left.\mathrm{CDCl}_{3}\right)$ (major Boc rotamer) $\delta 172.9(\mathrm{C}), 153.9(\mathrm{C}), 137.4(\mathrm{C}), 128.4(2 \times \mathrm{CH}), 127.8(2 \times$ 
$\mathrm{CH}), 127.8(\mathrm{CH}), 96.2\left(\mathrm{CH}_{2}\right), 86.2(\mathrm{C}), 80.8(\mathrm{C}), 79.7(\mathrm{CH}), 76.8(\mathrm{C}), 70.4\left(\mathrm{CH}_{2}\right), 65.3\left(\mathrm{CH}_{2}\right)$, $61.7\left(\mathrm{CH}_{2}\right), 53.0\left(\mathrm{CH}_{2}\right), 37.5(\mathrm{CH}), 28.2\left(3 \times \mathrm{CH}_{3}\right), 17.8\left(\mathrm{CH}_{3}\right), 14.0\left(\mathrm{CH}_{3}\right)$ and $9.6\left(\mathrm{CH}_{3}\right)$; (minor Boc rotamer) $\delta 172.7(\mathrm{C}), 152.6(\mathrm{C}), 137.2(\mathrm{C}), 128.5(2 \times \mathrm{CH}), 128.0(2 \times \mathrm{CH}), 128.0$ $(\mathrm{CH}), 95.9\left(\mathrm{CH}_{2}\right), 87.6(\mathrm{C}), 80.2(\mathrm{C}), 79.1(\mathrm{CH}), 77.2(\mathrm{C}), 70.5\left(\mathrm{CH}_{2}\right), 65.4\left(\mathrm{CH}_{2}\right), 61.5\left(\mathrm{CH}_{2}\right)$, $52.6\left(\mathrm{CH}_{2}\right), 37.0(\mathrm{CH}), 28.3\left(3 \times \mathrm{CH}_{3}\right), 17.6\left(\mathrm{CH}_{3}\right), 14.0\left(\mathrm{CH}_{3}\right)$ and $9.7\left(\mathrm{CH}_{3}\right)$; LRMS (ESI, $m / z) 526\left(\mathrm{M}+\mathrm{NH}_{4}{ }^{+}+\mathrm{MeCN}, 100 \%\right)$ and $490\left(\mathrm{M}+\mathrm{Na}^{+}, 15 \%\right)$; HRMS (ESI) calcd for $\mathrm{C}_{24} \mathrm{H}_{37} \mathrm{NNaO}_{8}\left(\mathrm{M}+\mathrm{Na}^{+}\right)$490.2417; found $490.2414(\Delta 0.5 \mathrm{ppm})$.

\section{Acetonide 17}
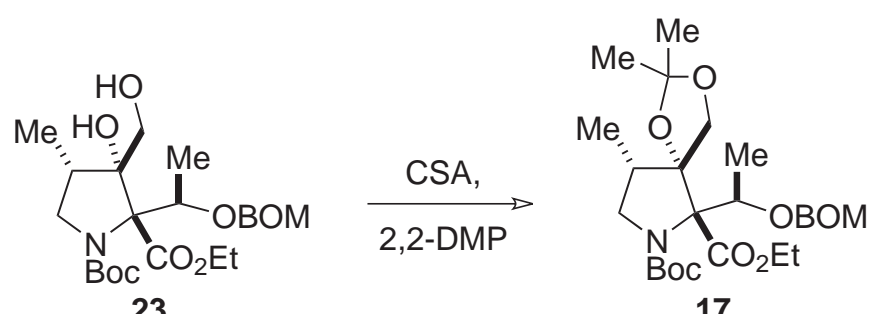

CSA (95.2 mg, $0.410 \mathrm{mmol}$ ) was added to a solution of crude 1,2-diol 23 (390 mg) in 2,2dimethoxypropane $(20 \mathrm{~mL})$ and the reaction mixture was stirred at $\mathrm{rt}$ for $39 \mathrm{~h}$. Aqueous $\mathrm{NaHCO}_{3}(5 \mathrm{~mL})$ was added and the layers were separated. The aqueous layer was extracted with EtOAc $(3 \times 20 \mathrm{~mL})$, dried with $\mathrm{MgSO}_{4}$ and evaporated under reduced pressure. The crude product was purified by flash column chromatography (13\% $\mathrm{Et}_{2} \mathrm{O}$-petrol) to give acetonide 17 (295 mg, $0.582 \mathrm{mmol}, 71 \%$ over 3 steps) as an oil.

$\boldsymbol{R}_{f}$ (40 \% Et $\mathrm{Et}_{2} \mathrm{O}$-petrol) 0.49; IR (thin film, $\mathrm{cm}^{-1}$ ) 2980, 2939, 1741, 1699, 1397, 1368, 1249 , 1170, 1040; ${ }^{1} \mathbf{H}$ NMR (400 MHz, $\left.\mathrm{CDCl}_{3}\right)$ ( 1.05:1 mixture of Boc rotamers) (major Boc rotamer) $\delta 7.35-7.22(\mathrm{~m}, 5 \mathrm{H}), 5.03(\mathrm{~d}, 1 \mathrm{H}, J=7.1 \mathrm{~Hz}), 4.85(\mathrm{~d}, 1 \mathrm{H}, J=7.1 \mathrm{~Hz}), 4.77(\mathrm{~d}, 1 \mathrm{H}$, $J=11.6 \mathrm{~Hz}), 4.67(\mathrm{q}, 1 \mathrm{H}, J=6.8 \mathrm{~Hz}), 4.46(\mathrm{~d}, 1 \mathrm{H}, J=11.6 \mathrm{~Hz}), 4.28(\mathrm{~d}, 1 \mathrm{H}, J=8.6 \mathrm{~Hz})$, $4.23-4.05(\mathrm{~m}, 2 \mathrm{H}), 3.77(\mathrm{~d}, 1 \mathrm{H}, J=8.6 \mathrm{~Hz}), 3.74(\mathrm{dd}, 1 \mathrm{H}, J=10.4$ and $7.3 \mathrm{~Hz}), 2.97(\mathrm{dd}, 1 \mathrm{H}$, $J=10.4$ and $5.8 \mathrm{~Hz}), 2.55-2.42(\mathrm{~m}, 1 \mathrm{H}), 1.63(\mathrm{~s}, 3 \mathrm{H}), 1.52(\mathrm{~s}, 3 \mathrm{H}), 1.44(\mathrm{~d}, 3 \mathrm{H}, J=6.8 \mathrm{~Hz})$, $1.43(\mathrm{~s}, 9 \mathrm{H}), 1.26(\mathrm{t}, 3 \mathrm{H}, J=7.1 \mathrm{~Hz})$ and $1.06(\mathrm{~d}, 3 \mathrm{H}, J=6.3 \mathrm{~Hz})$; (minor Boc rotamer) 7.35$7.22(\mathrm{~m}, 5 \mathrm{H}), 5.05(\mathrm{~d}, 1 \mathrm{H}, J=6.9 \mathrm{~Hz}), 4.89-4.82(\mathrm{~m}, 2 \mathrm{H}), 4.77(\mathrm{~d}, 1 \mathrm{H}, J=11.6 \mathrm{~Hz}), 4.46(\mathrm{~d}$, $1 \mathrm{H}, J=11.6 \mathrm{~Hz}), 4.40(\mathrm{~d}, 1 \mathrm{H}, J=8.6 \mathrm{~Hz}), 4.23-4.05(\mathrm{~m}, 2 \mathrm{H}), 3.89(\mathrm{dd}, 1 \mathrm{H}, J=10.4$ and $7.3 \mathrm{~Hz}), 3.79(\mathrm{~d}, 1 \mathrm{H}, J=8.6 \mathrm{~Hz}), 3.00(\mathrm{dd}, 1 \mathrm{H}, J=10.4$ and $5.8 \mathrm{~Hz}), 2.55-2.42(\mathrm{~m}, 1 \mathrm{H}), 1.61$ $(\mathrm{s}, 3 \mathrm{H}), 1.51(\mathrm{~s}, 3 \mathrm{H}), 1.46(\mathrm{~d}, 3 \mathrm{H}, J=6.8 \mathrm{~Hz}), 1.39(\mathrm{~s}, 9 \mathrm{H}), 1.24(\mathrm{t}, 3 \mathrm{H}, J=7.1 \mathrm{~Hz})$ and 1.04 $(\mathrm{d}, 3 \mathrm{H}, J=6.3 \mathrm{~Hz}) ;{ }^{13} \mathrm{C}$ NMR $\left(100 \mathrm{MHz}, \mathrm{CDCl}_{3}\right)$ (major Boc rotamer) $\delta 171.6(\mathrm{C}), 152.8$ (C), $138.0(\mathrm{C}), 128.4(2 \times \mathrm{CH}), 127.7(2 \times \mathrm{CH}), 127.6(\mathrm{CH}), 112.4(\mathrm{C}), 96.6\left(\mathrm{CH}_{2}\right), 93.7(\mathrm{C})$, $79.8(\mathrm{C}), 78.5(\mathrm{CH}), 74.0(\mathrm{C}), 71.2\left(\mathrm{CH}_{2}\right), 69.7\left(\mathrm{CH}_{2}\right), 61.3\left(\mathrm{CH}_{2}\right), 52.4\left(\mathrm{CH}_{2}\right), 40.7(\mathrm{CH})$, $28.2\left(3 \times \mathrm{CH}_{3}\right), 27.3\left(\mathrm{CH}_{3}\right), 26.7\left(\mathrm{CH}_{3}\right), 17.8\left(\mathrm{CH}_{3}\right), 14.1\left(\mathrm{CH}_{3}\right)$ and $11.7\left(\mathrm{CH}_{3}\right)$; (minor Boc 
rotamer) $\delta 171.8(\mathrm{C}), 153.6(\mathrm{C}), 138.3(\mathrm{C}), 128.3(2 \times \mathrm{CH}), 127.9(2 \times \mathrm{CH}), 127.4(\mathrm{CH})$, $112.2(\mathrm{C}), 96.6\left(\mathrm{CH}_{2}\right), 92.5(\mathrm{C}), 80.8(\mathrm{C}), 77.8(\mathrm{CH}), 74.0(\mathrm{C}), 71.1\left(\mathrm{CH}_{2}\right), 69.5\left(\mathrm{CH}_{2}\right), 61.2$ $\left(\mathrm{CH}_{2}\right), 52.9\left(\mathrm{CH}_{2}\right), 41.0(\mathrm{CH}), 28.4\left(3 \times \mathrm{CH}_{3}\right), 27.3\left(\mathrm{CH}_{3}\right), 26.6\left(\mathrm{CH}_{3}\right), 18.9\left(\mathrm{CH}_{3}\right), 14.1\left(\mathrm{CH}_{3}\right)$ and $11.7\left(\mathrm{CH}_{3}\right)$; LRMS (ESI, $\left.\mathrm{m} / \mathrm{z}\right) 566\left(\mathrm{M}+\mathrm{NH}_{4}{ }^{+}+\mathrm{MeCN}, 100 \%\right)$; HRMS (ESI) calcd for $\mathrm{C}_{27} \mathrm{H}_{41} \mathrm{NNaO}_{8}\left(\mathrm{M}+\mathrm{Na}^{+}\right)$530.2724; found 530.2732 ( $\left.\triangle 1.4 \mathrm{ppm}\right)$.

\section{$N$-Boc lactam 18}

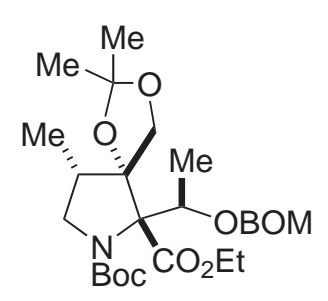

17

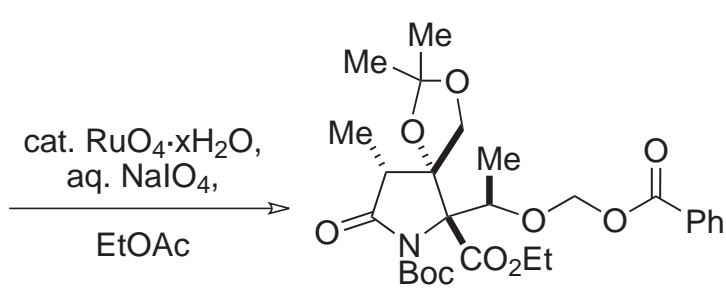

18

$10 \%$ aqueous $\mathrm{NaIO}_{4}$ solution $(3 \mathrm{~mL})$ was added to $\mathrm{RuO}_{2} \cdot x \mathrm{H}_{2} \mathrm{O}(20.0 \mathrm{mg})$ to give a yellow solution. A solution of acetonide $17(71.7 \mathrm{mg}, 0.141 \mathrm{mmol})$ in EtOAc $(3 \mathrm{~mL})$ was added dropwise and the resulting biphasic mixture was stirred at $40{ }^{\circ} \mathrm{C}$ for $20 \mathrm{~h}$. The black reaction mixture was treated with more $10 \% \mathrm{NaIO}_{4}$ solution until it turned yellow. The layers were separated and the aqueous layer was extracted with EtOAc $(3 \times 15 \mathrm{~mL})$. The combined organic extracts were treated with 2-propanol $(0.5 \mathrm{~mL})$, stirred for $30 \mathrm{~min}$ and filtered through Celite ${ }^{\circledR}$. The filtrate was dried with $\mathrm{MgSO}_{4}$, filtered and evaporated under reduced pressure. The residue was purified by flash column chromatography (13.5\% $\mathrm{Et}_{2} \mathrm{O}$-petrol) to give lactam 18 (49.6 mg, $92.7 \mu \mathrm{mol}, 66 \%)$ as an oil.

$\boldsymbol{R}_{\boldsymbol{f}}\left(40 \% \mathrm{Et}_{2} \mathrm{O}\right.$-petrol) 0.22; IR (thin film, $\mathrm{cm}^{-1}$ ) 2928, 1794, 1756, 1738, 1730, 1454, 1371, 1254, 1155, 1058; ${ }^{1} \mathbf{H}$ NMR (400 MHz, $\left.\mathrm{CDCl}_{3}\right): \delta$ 8.10-8.07 (m, 2H), 7.62-7.57 (m, 1H), $7.50-7.44(\mathrm{~m}, 2 \mathrm{H}), 5.85(\mathrm{~d}, 1 \mathrm{H}, J=6.6 \mathrm{~Hz}), 5.59(\mathrm{~d}, 1 \mathrm{H}, J=6.6 \mathrm{~Hz}), 4.91(\mathrm{q}, 1 \mathrm{H}, J=6.6 \mathrm{~Hz})$, $4.49(\mathrm{~d}, 1 \mathrm{H}, J=9.1 \mathrm{~Hz}), 4.25(\mathrm{dq}, 2 \mathrm{H}, J=10.9$ and $7.1 \mathrm{~Hz}), 4.22(\mathrm{dq}, 2 \mathrm{H}, J=10.9$ and $7.1 \mathrm{~Hz}), 3.77(\mathrm{~d}, 1 \mathrm{H}, J=9.1 \mathrm{~Hz}), 2.82(\mathrm{q}, 1 \mathrm{H}, J=7.2 \mathrm{~Hz}), 1.53(\mathrm{~s}, 9 \mathrm{H}), 1.51(\mathrm{~s}, 3 \mathrm{H}), 1.43(\mathrm{~d}$, $3 \mathrm{H}, J=6.6 \mathrm{~Hz}), 1.39(\mathrm{~s}, 3 \mathrm{H}), 1.30(\mathrm{t}, 3 \mathrm{H}, J=7.1 \mathrm{~Hz})$ and $1.29(\mathrm{~d}, 3 \mathrm{H}, J=7.2 \mathrm{~Hz}) ;{ }^{13} \mathbf{C ~ N M R}$ $\left(100 \mathrm{MHz}, \mathrm{CDCl}_{3}\right.$ ) (major Boc rotamer) $\delta 174.6(\mathrm{C}), 169.6(\mathrm{C}), 165.8(\mathrm{C}), 150.1(\mathrm{C}), 133.2$ $(\mathrm{CH}), 130.0(\mathrm{C}), 129.7(2 \times \mathrm{CH}), 128.5(2 \times \mathrm{CH}), 112.9(\mathrm{C}), 89.8\left(\mathrm{CH}_{2}\right), 85.4(\mathrm{C}), 84.1(\mathrm{C})$, $78.1(\mathrm{CH}), 73.7(\mathrm{C}), 72.5\left(\mathrm{CH}_{2}\right), 62.1\left(\mathrm{CH}_{2}\right), 46.7(\mathrm{CH}), 27.9\left(3 \times \mathrm{CH}_{3}\right), 26.5\left(\mathrm{CH}_{3}\right), 26.3$ $\left(\mathrm{CH}_{3}\right), 17.8\left(\mathrm{CH}_{3}\right), 14.0\left(\mathrm{CH}_{3}\right)$ and $10.5\left(\mathrm{CH}_{3}\right)$; LRMS (ESI, $\left.\mathrm{m} / z\right) 594\left(\mathrm{M}+\mathrm{NH}_{4}{ }^{+}+\mathrm{MeCN}\right.$, $100 \%)$; HRMS (ESI) calcd for $\mathrm{C}_{27} \mathrm{H}_{37} \mathrm{NNaO}_{10}\left(\mathrm{M}+\mathrm{Na}^{+}\right)$; found $558.2300(\Delta 1.7 \mathrm{ppm})$. 


\section{Lactam 24}

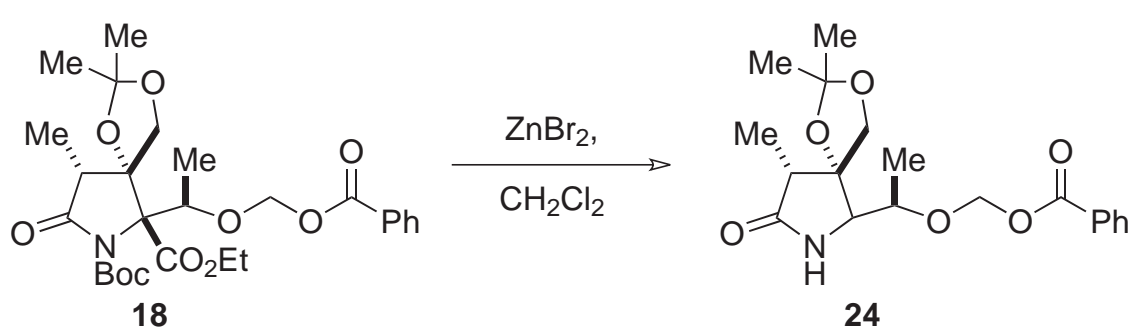

$\mathrm{ZnBr}_{2}(113 \mathrm{mg}, 0.502 \mathrm{mmol})$ was dried at $200{ }^{\circ} \mathrm{C}$ under vacuum for $4 \mathrm{~h}$. Argon was introduced upon cooling and $\mathrm{CH}_{2} \mathrm{Cl}_{2}(4 \mathrm{~mL})$ was added. A solution of N-Boc lactam 18 (80.8 $\mathrm{mg}, 0.151 \mathrm{mmol})$ in $\mathrm{CH}_{2} \mathrm{Cl}_{2}(6 \mathrm{~mL})$ was added dropwise and the reaction mixture was stirred for $15 \mathrm{~h}$. The reaction was quenched with water and the layers were separated. The aqueous layer was extracted with EtOAc $(3 \times 20 \mathrm{~mL})$. The combined organic layers were washed with brine, dried with $\mathrm{MgSO}_{4}$ and evaporated under reduced pressure. The residue was subjected to column chromatography $\left(13.5 \% \mathrm{Me}_{2} \mathrm{CO}\right.$-petrol) to give the deprotected lactam $24(65.1 \mathrm{mg})$ as an oil which solidified on standing, containing trace amounts of impurities but was not purified further at this stage.

$\boldsymbol{R}_{\boldsymbol{f}}$ (40\% $\mathrm{Me}_{2} \mathrm{CO}$-petrol) 0.58 ; $\mathbf{m p}$ decomposed at $80{ }^{\circ} \mathrm{C}$; IR (thin film, $\mathrm{cm}^{-1}$ ) 3215, 2985, 1720, 1711, 1453, 1383, 1269, 1132, 1058; ${ }^{1} \mathbf{H}$ NMR (400 MHz, $\left.\mathrm{CDCl}_{3}\right) \delta 8.08-8.05$ (m, 2H), 7.61-7.55 (m, 1H), 7.48-7.43 (m, 2H), 6.73 (br s, 1H,), 5.68 (d, 1H, J=6.6 Hz), 5.53 (d, $1 \mathrm{H}$, $J=6.6 \mathrm{~Hz}), 4.44(\mathrm{q}, 1 \mathrm{H}, J=6.6 \mathrm{~Hz}), 4.28(\mathrm{~d}, 1 \mathrm{H}, J=10.1 \mathrm{~Hz}), 4.23(\mathrm{qd}, 2, J=7.3$ and $3.0 \mathrm{~Hz}), 3.80(\mathrm{~d}, 1 \mathrm{H}, J=10.1 \mathrm{~Hz}), 2.40(\mathrm{q}, 1 \mathrm{H}, J=7.3 \mathrm{~Hz}), 1.43(\mathrm{~s}, 3 \mathrm{H}), 1.30(\mathrm{t}, 3 \mathrm{H}$, $J=7.3 \mathrm{~Hz}), 1.28(\mathrm{~s}, 3 \mathrm{H}), 1.26(\mathrm{~d}, 3 \mathrm{H}, J=6.6 \mathrm{~Hz})$ and $1.25(\mathrm{~d}, 3 \mathrm{H}, J=7.3 \mathrm{~Hz}) ;{ }^{13} \mathbf{C ~ N M R}$ $\left(100 \mathrm{MHz}, \mathrm{CDCl}_{3}\right) \delta 177.0(\mathrm{C}), 170.1(\mathrm{C}), 165.9(\mathrm{C}), 133.4(\mathrm{CH}), 129.8(2 \times \mathrm{CH}), 129.8(\mathrm{C})$, $128.5(2 \times \mathrm{CH}), 111.3(\mathrm{C}), 88.7\left(\mathrm{CH}_{2}\right), 86.8(\mathrm{C}), 76.0(\mathrm{CH}), 75.1(\mathrm{C}), 74.3\left(\mathrm{CH}_{2}\right), 62.1\left(\mathrm{CH}_{2}\right)$, $46.1(\mathrm{CH}), 26.0\left(\mathrm{CH}_{3}\right), 25.3\left(\mathrm{CH}_{3}\right), 16.3\left(\mathrm{CH}_{3}\right), 14.0\left(\mathrm{CH}_{3}\right)$ and $11.0\left(\mathrm{CH}_{3}\right)$; LRMS (ESI, $\left.\mathrm{m} / \mathrm{z}\right)$ $494\left(\mathrm{M}+\mathrm{NH}_{4}{ }^{+}+\mathrm{MeCN}, 100 \%\right)$; HRMS (ESI) calcd for $\mathrm{C}_{22} \mathrm{H}_{29} \mathrm{NNaO}_{8}\left(\mathrm{M}+\mathrm{Na}^{+}\right) 458.1785$; found $458.1785(\triangle 0.1 \mathrm{ppm})$. 


\section{$N$-methylated lactam 19}
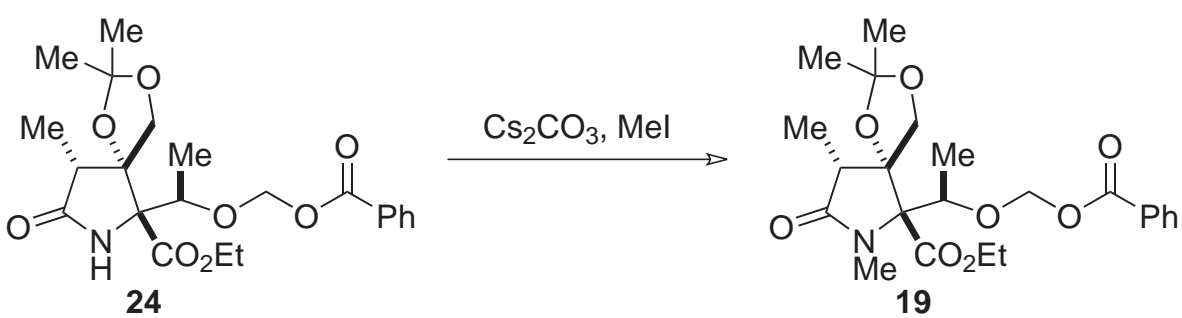

Lactam $24(65.1 \mathrm{mg})$ was stirred with $\mathrm{Cs}_{2} \mathrm{CO}_{3}(195 \mathrm{mg}, 0.598 \mathrm{mmol})$ and $\mathrm{MeI}(2 \mathrm{~mL})$ for $24 \mathrm{~h}$, after which more $\mathrm{Cs}_{2} \mathrm{CO}_{3}(145 \mathrm{mg}, 0.445 \mathrm{mmol})$ was added. Stirring was continued for $18 \mathrm{~h}$ and the reaction was quenched with $\mathrm{H}_{2} \mathrm{O}(2 \mathrm{~mL})$ and diluted with EtOAc $(10 \mathrm{~mL})$. The layers were separated and the aqueous layer was extracted with EtOAc $(3 \times 15 \mathrm{~mL})$. The combined organic layers were washed with brine, washed with $\mathrm{MgSO}_{4}$ and evaporated under reduced pressure. The residue was subjected to column chromatography ( $7 \% \mathrm{Me}_{2} \mathrm{CO}$-petrol) to give the $N$-methylated lactam $19(59.4 \mathrm{mg})$ as an oil containing trace amounts of impurities but was not purified further at this stage.

$\boldsymbol{R}_{\boldsymbol{f}}\left(30 \% \mathrm{Me}_{2} \mathrm{CO}\right.$-petrol) 0.46; IR (thin film, $\mathrm{cm}^{-1}$ ) 2984, 2927, 2361, 2341, 1733, 1703, 1382, 1268, 1242, 1058; ${ }^{1} \mathbf{H}$ NMR $\left(400 \mathrm{MHz}, \mathrm{CDCl}_{3}\right) \delta 8.09-8.04(\mathrm{~m}, 2 \mathrm{H}), 7.61-7.55(\mathrm{~m}, 1 \mathrm{H})$, 7.48-7.42 (m, 2H), $5.74(\mathrm{~d}, 1 \mathrm{H}, J=6.5 \mathrm{~Hz}), 5.48(\mathrm{~d}, 1 \mathrm{H}, J=6.5 \mathrm{~Hz}), 4.63(\mathrm{q}, 1 \mathrm{H}$, $J=6.7 \mathrm{~Hz}), 4.32-4.14(\mathrm{~m}, 3 \mathrm{H}), 3.79(\mathrm{~d}, 1 \mathrm{H}, J=9.9 \mathrm{~Hz}),, 2.92(\mathrm{~s}, 3 \mathrm{H}), 2.44(\mathrm{q}, 1 \mathrm{H}$, $J=7.5 \mathrm{~Hz}), 1.42(\mathrm{~d}, 3 \mathrm{H}, J=6.7 \mathrm{~Hz}), 1.39(\mathrm{~s}, 3 \mathrm{H}), 1.29(\mathrm{~d}, 3 \mathrm{H}, J=7.5 \mathrm{~Hz}), 1.29(\mathrm{t}, 3 \mathrm{H}$, $J=7.2 \mathrm{~Hz})$ and $1.26(\mathrm{~s}, 3 \mathrm{H}) ;{ }^{13} \mathrm{C}$ NMR $\left(100 \mathrm{MHz}, \mathrm{CDCl}_{3}\right) \delta 176.4(\mathrm{C}), 169.6(\mathrm{C}), 165.8(\mathrm{C})$, $133.4(\mathrm{CH}), 129.8(\mathrm{C}), 129.8(2 \times \mathrm{CH}), 128.5(2 \times \mathrm{CH}), 111.4(\mathrm{C}), 88.3\left(\mathrm{CH}_{2}\right), 85.7(\mathrm{C}), 75.7$ $\left(\mathrm{CH}_{2}\right), 75.5(\mathrm{CH}), 75.2(\mathrm{C}), 61.8\left(\mathrm{CH}_{2}\right), 45.9(\mathrm{CH}), 30.1\left(\mathrm{CH}_{3}\right), 26.0\left(\mathrm{CH}_{3}\right), 25.3\left(\mathrm{CH}_{3}\right), 17.8$ $\left(\mathrm{CH}_{3}\right), 14.0\left(\mathrm{CH}_{3}\right)$ and $11.9\left(\mathrm{CH}_{3}\right)$; LRMS (ESI, $\left.\mathrm{m} / z\right) 508\left(\mathrm{M}+\mathrm{NH}_{4}{ }^{+}+\mathrm{MeCN}, 100 \%\right)$ and $472\left(\mathrm{M}+\mathrm{Na}^{+}, 5 \%\right)$; HRMS (ESI) calcd for $\mathrm{C}_{23} \mathrm{H}_{31} \mathrm{NNaO}_{8}\left(\mathrm{M}+\mathrm{Na}^{+}\right)$472.1942; found $472.1945(\Delta 0.7 \mathrm{ppm})$.

\section{1,3-Diol 20}
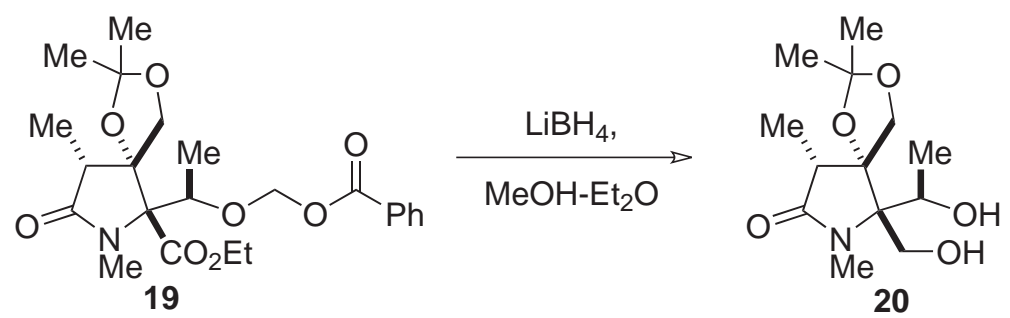

A flame-dried flask was charged with $\mathrm{LiBH}_{4}(14.4 \mathrm{mg}, 0.661 \mathrm{mmol})$ and flushed with argon. $\mathrm{Et}_{2} \mathrm{O}(4 \mathrm{~mL})$ was added, followed by $\mathrm{MeOH}(27 \mu \mathrm{L}, 0.661 \mathrm{mmol})$. A solution of $N$-methylated lactam 19 (59.4 mg) in $\mathrm{Et}_{2} \mathrm{O}(12 \mathrm{~mL})$ was added dropwise and the reaction mixture was stirred 
at $\mathrm{rt}$ for $5 \mathrm{~h}$. The reaction was quenched with $\mathrm{H}_{2} \mathrm{O}(4 \mathrm{~mL})$ and diluted with EtOAc. The layers were separated and the aqueous layer was extracted with EtOAc $(3 \times 25 \mathrm{~mL})$. The combined organic extracts were dried with $\mathrm{Na}_{2} \mathrm{SO}_{4}$, filtered and evaporated under reduced pressure. The crude product was purified by column chromatography $\left(10 \% \rightarrow 30 \% \mathrm{Me}_{2} \mathrm{CO}\right.$-petrol) to give 1,3-diol 20 (31.3 mg, $0.115 \mathrm{mmol}, 76 \%$ over 3 steps) as needles.

$\boldsymbol{R}_{f}$ (20\% Me $\mathrm{Me}_{2} \mathrm{CO}$-petrol) 0.28; mp 167-169 ${ }^{\circ} \mathrm{C}, \mathbf{I R}\left(\mathrm{KBr}\right.$ disc, $\left.\mathrm{cm}^{-1}\right)$ 3406, 2988, 2931, 1669 , 1457, 1386, 1247, 1214, 1167, 1051; ${ }^{1} \mathbf{H}$ NMR $\left(400 \mathrm{MHz}, \mathrm{CDCl}_{3}\right) \delta 5.11(\mathrm{~s}, 1 \mathrm{H}), 4.36(\mathrm{~d}, 1 \mathrm{H}$, $J=10.5 \mathrm{~Hz}), 4.13(\mathrm{~d}, 1 \mathrm{H}, J=10.5 \mathrm{~Hz}), 4.09(\mathrm{qd}, 1 \mathrm{H}, J=6.9$ and $1.3 \mathrm{~Hz}), 3.93(\mathrm{dd}, 1 \mathrm{H}$, $J=13.0$ and $4.9 \mathrm{~Hz}), 3.71(\mathrm{~s}, \mathrm{H}), 3.42(\mathrm{dd}, 1 \mathrm{H}, J=13.0$ and $8.1 \mathrm{~Hz}), 2.95(\mathrm{~s}, 3 \mathrm{H}), 2.78(\mathrm{q}, 1 \mathrm{H}$, $J=7.4 \mathrm{~Hz}), 1.52(\mathrm{~s}, 3 \mathrm{H}), 1.45(\mathrm{~s}, 3 \mathrm{H}), 1.27(\mathrm{~d}, 3 \mathrm{H}, J=6.9 \mathrm{~Hz})$ and $1.12(\mathrm{~d}, 3 \mathrm{H}, J=7.4 \mathrm{~Hz})$; ${ }^{13}$ C NMR (100 MHz, $\left.\mathrm{CDCl}_{3}\right) \delta 176.5(\mathrm{C}), 110.4(\mathrm{C}), 89.9(\mathrm{C}), 73.2(\mathrm{C}), 69.1\left(\mathrm{CH}_{2}\right), 66.4$ $(\mathrm{CH}), 60.4\left(\mathrm{CH}_{2}\right), 45.2(\mathrm{CH}), 27.5\left(\mathrm{CH}_{3}\right), 25.7\left(\mathrm{CH}_{3}\right), 25.7\left(\mathrm{CH}_{3}\right), 17.5\left(\mathrm{CH}_{3}\right)$ and $10.2\left(\mathrm{CH}_{3}\right)$; LRMS (ESI, $m / z) 332\left(\mathrm{M}+\mathrm{NH}_{4}{ }^{+}+\mathrm{MeCN}, 100 \%\right)$; HRMS (ESI) calcd for $\mathrm{C}_{13} \mathrm{H}_{23} \mathrm{NNaO}_{5}$ $\left(\mathrm{M}+\mathrm{Na}^{+}\right) 296.1468$; found $296.1466(\Delta 0.8 \mathrm{ppm})$.

\section{Hydroxyaldehyde 25}
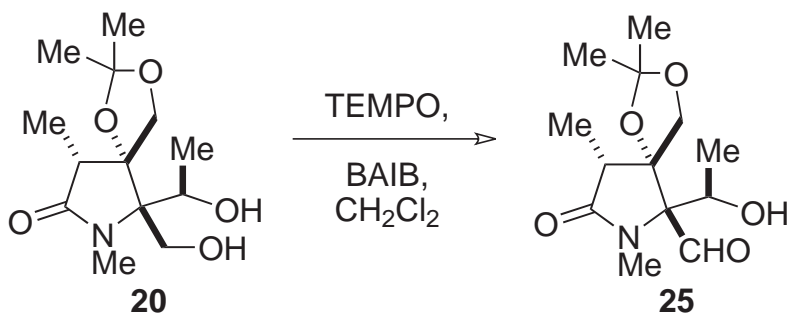

BAIB $(24.7 \mathrm{mg}, 76.6 \mu \mathrm{mol})$ and TEMPO $(2.4 \mathrm{mg}, 15.3 \mu \mathrm{mol})$ were added to a stirred solution of diol $20(19.0 \mathrm{mg}, 69.5 \mu \mathrm{mol})$ in wet $\mathrm{CH}_{2} \mathrm{Cl}_{2}(3 \mathrm{~mL})$. The reaction mixture was stirred for 24 $\mathrm{h}$ and quenched with saturated $\mathrm{Na}_{2} \mathrm{~S}_{2} \mathrm{O}_{3}(5 \mathrm{~mL})$ and poured into $\mathrm{CH}_{2} \mathrm{Cl}_{2}(10 \mathrm{~mL})$. The layers were separated and the aqueous layer was extracted with $\mathrm{CH}_{2} \mathrm{Cl}_{2}(3 \times 10 \mathrm{~mL})$. The combined organic extracts were washed with aqueous $\mathrm{NaHCO}_{3}(10 \mathrm{~mL})$ and brine $(10 \mathrm{~mL})$, dried with $\mathrm{Na}_{2} \mathrm{SO}_{4}$, filtered and evaporated under reduced pressure to give crude hydroxyaldehyde 25 $(17.5 \mathrm{mg}, 64.6 \mu \mathrm{mol}, 93 \%)$ as an oil which did not require any further purification.

$\boldsymbol{R}_{f}\left(20 \% \mathrm{Me}_{2} \mathrm{CO}\right.$-petrol) 0.36; IR (thin film, $\mathrm{cm}^{-1}$ ) 3520, 2988, 2924, 2852, 1726, 1694, 1455, 1430, 1385, 1059; ${ }^{1} \mathbf{H}$ NMR (500 MHz, $\left.\mathrm{CDCl}_{3}\right) \delta 9.68(\mathrm{~s}, 1 \mathrm{H}), 4.63(\mathrm{q}, 1 \mathrm{H}, J=6.8 \mathrm{~Hz}), 4.42$ $(\mathrm{d}, 1 \mathrm{H}, J=10.4 \mathrm{~Hz}), 3.93(\mathrm{~d}, 1 \mathrm{H}, J=10.4 \mathrm{~Hz}), 3.58(\mathrm{br} \mathrm{s}, 1 \mathrm{H}), 3.10$ (s, 3H), 2.21 (q, 1H, $J=7.1 \mathrm{~Hz}), 1.59(\mathrm{~s}, 3 \mathrm{H}), 1.49(\mathrm{~s}, 3 \mathrm{H}), 1.42(\mathrm{~d}, 3 \mathrm{H}, J=6.8 \mathrm{~Hz})$ and $1.24(\mathrm{~d}, 3 \mathrm{H}, J=7.1 \mathrm{~Hz})$; ${ }^{13}$ C NMR (125 MHz, $\left.\mathrm{CDCl}_{3}\right) \delta 199.3$ (C), 175.0 (C), 111.3 (C), 88.9 (C), 78.2 (C), 67.7 
$\left(\mathrm{CH}_{2}\right), 65.8(\mathrm{CH}), 44.2(\mathrm{CH}), 29.0\left(\mathrm{CH}_{3}\right), 25.7\left(\mathrm{CH}_{3}\right), 25.5\left(\mathrm{CH}_{3}\right), 18.4\left(\mathrm{CH}_{3}\right)$ and $9.4\left(\mathrm{CH}_{3}\right)$; LRMS (ESI, m/z) $362\left(\mathrm{M}+\mathrm{MeOH}+\mathrm{NH}_{4}{ }^{+}+\mathrm{MeCN}, 100 \%\right)$; HRMS (FI) calcd for $\mathrm{C}_{13} \mathrm{H}_{22} \mathrm{NO}_{5}\left(\mathrm{M}+\mathrm{H}^{+}\right)$272.1498; found $272.1493(\Delta 1.7 \mathrm{ppm})$.

\section{Lactone 3}

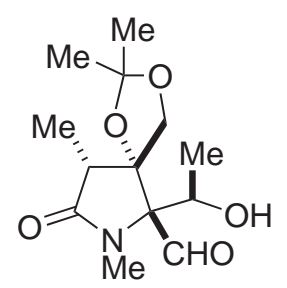

25

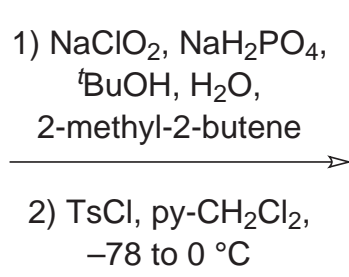

-78 to $0^{\circ} \mathrm{C}$

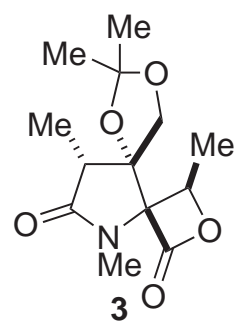

Hydroxyaldehyde $25(17.5 \mathrm{mg}, 64.6 \mu \mathrm{mol})$ was dissolved in ${ }^{t} \mathrm{BuOH}(4.5 \mathrm{~mL})$ and 2-methyl-2butene $(1.5 \mathrm{~mL})$ and cooled to $0{ }^{\circ} \mathrm{C}$. A solution of $\mathrm{NaClO}_{2}(58.4 \mathrm{mg}, 0.646 \mathrm{mmol})$ and $\mathrm{NaH}_{2} \mathrm{PO}_{4}(62.0 \mathrm{mg}, 0.517 \mathrm{mmol})$ in $\mathrm{H}_{2} \mathrm{O}(3 \mathrm{~mL})$ was added dropwise and the reaction mixture was warmed to $\mathrm{rt}$ and stirred vigorously for $3.5 \mathrm{~h}$. The reaction mixture was diluted with $\mathrm{H}_{2} \mathrm{O}$ $(3 \mathrm{~mL})$ and was washed once with EtOAc $(10 \mathrm{~mL})$. The aqueous layer was acidified to $\mathrm{pH} 3$ and extracted with EtOAc $(6 \times 10 \mathrm{~mL})$. The combined organic extracts were dried with $\mathrm{Na}_{2} \mathrm{SO}_{4}$, filtered and evaporated under reduced pressure to give the crude hydroxyacid 4 (10.5 mg). This was dissolved in 1:1 pyridine- $\mathrm{CH}_{2} \mathrm{Cl}_{2}(2 \mathrm{~mL})$ and cooled to $-5{ }^{\circ} \mathrm{C}$. TsCl $(22.0 \mathrm{mg}, 0.115 \mathrm{mmol}$ ) was added and upon dissolution, the reaction mixture was warmed to $0{ }^{\circ} \mathrm{C}$ and stirred for $20 \mathrm{~h}$. The reaction mixture was diluted with $\mathrm{H}_{2} \mathrm{O}(2 \mathrm{~mL})$ and poured into EtOAc $(5 \mathrm{~mL})$. The layers were separated and the aqueous layer was extracted with EtOAc $(3 \times 10 \mathrm{~mL})$. The combined organic extracts were dried with $\mathrm{Na}_{2} \mathrm{SO}_{4}$, filtered and evaporated under reduced pressure. The pyridine was azeotropically removed with $\mathrm{PhMe}$. The crude product was purified by gradient flash column chromatography $\left(5 \% \rightarrow 9 \% \mathrm{Me}_{2} \mathrm{CO}\right.$-petrol) to give lactone $\mathbf{3}$ ( $6.9 \mathrm{mg}, 25.7 \mu \mathrm{mol}, 40 \%$ over 2 steps) as needles.

$\boldsymbol{R}_{\boldsymbol{f}}$ (20\% $\mathrm{Me}_{2} \mathrm{CO}$-petrol) 0.28; mp 146-149 ${ }^{\circ} \mathrm{C}$; IR (KBr disc, $\left.\mathrm{cm}^{-1}\right)$ 2948, 1819, 1720, 1463, 1378, 1287, 1272, 1184, 1029; ${ }^{1} \mathbf{H}$ NMR $\left(500 \mathrm{MHz}, \mathrm{CDCl}_{3}\right) \delta 4.83(\mathrm{q}, 1 \mathrm{H}, J=6.7 \mathrm{~Hz}), 4.77$ $(\mathrm{d}, 1 \mathrm{H}, J=9.4 \mathrm{~Hz}), 3.81(\mathrm{~d}, 1 \mathrm{H}, J=9.4 \mathrm{~Hz}), 2.91(\mathrm{~s}, 3 \mathrm{H}), 2.41(\mathrm{q}, 1 \mathrm{H}, J=7.5 \mathrm{~Hz}), 1.83$ (d, $3 \mathrm{H}, J=6.7 \mathrm{~Hz}), 1.52(\mathrm{~s}, 3 \mathrm{H}), 1.42(\mathrm{~s}, 3 \mathrm{H})$ and $1.24(\mathrm{~d}, 3 \mathrm{H}, J=7.5 \mathrm{~Hz}) ;{ }^{13} \mathbf{C} \mathbf{N M R}(125 \mathrm{MHz}$, $\left.\mathrm{CDCl}_{3}\right) \delta 174.4(\mathrm{C}), 167.4(\mathrm{C}), 111.9(\mathrm{C}), 85.0(\mathrm{C}), 81.5(\mathrm{C}), 78.7(\mathrm{CH}), 73.1\left(\mathrm{CH}_{2}\right), 45.2$ (CH), $26.4\left(\mathrm{CH}_{3}\right), 26.1\left(\mathrm{CH}_{3}\right), 25.7\left(\mathrm{CH}_{3}\right), 16.4\left(\mathrm{CH}_{3}\right)$ and $13.6\left(\mathrm{CH}_{3}\right)$; LRMS (ESI, $\left.m / z\right) 292$ $\left(\mathrm{M}+\mathrm{Na}^{+}, 100 \%\right)$; HRMS (ESI) calcd for $\mathrm{C}_{13} \mathrm{H}_{19} \mathrm{NNaO}_{5}\left(\mathrm{M}+\mathrm{Na}^{+}\right) 292.1155$; found 292.1149 $(\Delta 2.1 \mathrm{ppm})$. 


\section{References}

1. Donohoe, T. J.; Sintim, H. O.; Sisangia, L.; Harling, J. D. Angew. Chem., Int. Ed. 2004, 43, 2293.

2. Donohoe, T. J.; House, D.; Ace, K. W. Org. Biomol. Chem. 2003, 1, 3749. 


\section{${ }^{13} \mathrm{C}$ NMR of $N$-Boc pyrrole 8}
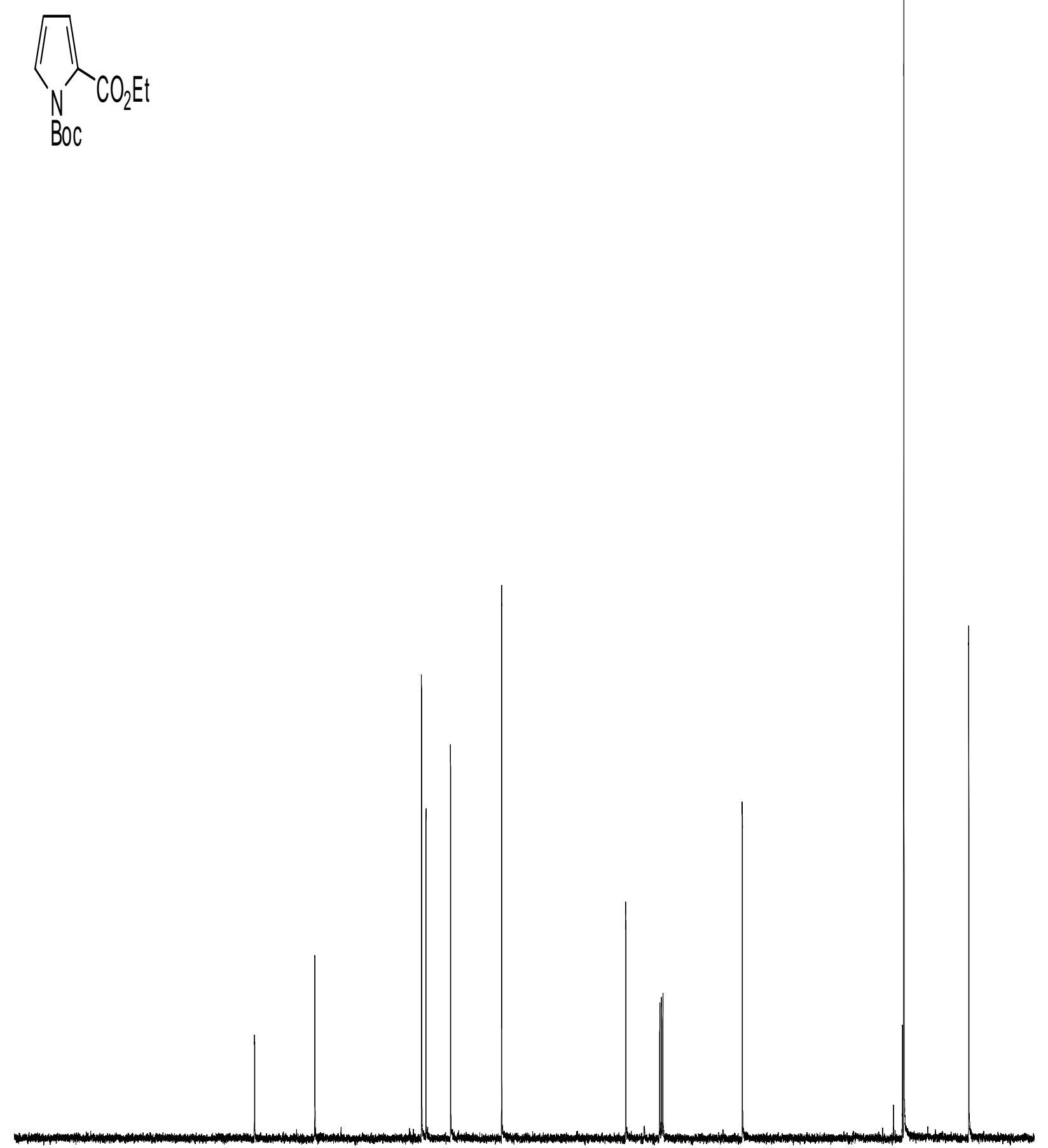



$\begin{array}{llllllllllllllllllll}200 & 190 & 180 & 170 & 160 & 150 & 140 & 130 & 120 & 110 & 100 & 90 & 80 & 70 & 60 & 50 & 40 & 30 & 20 & 10\end{array}$ chemical shift (ppm) 
${ }^{13} \mathrm{C}$ NMR of anti-aldol 10
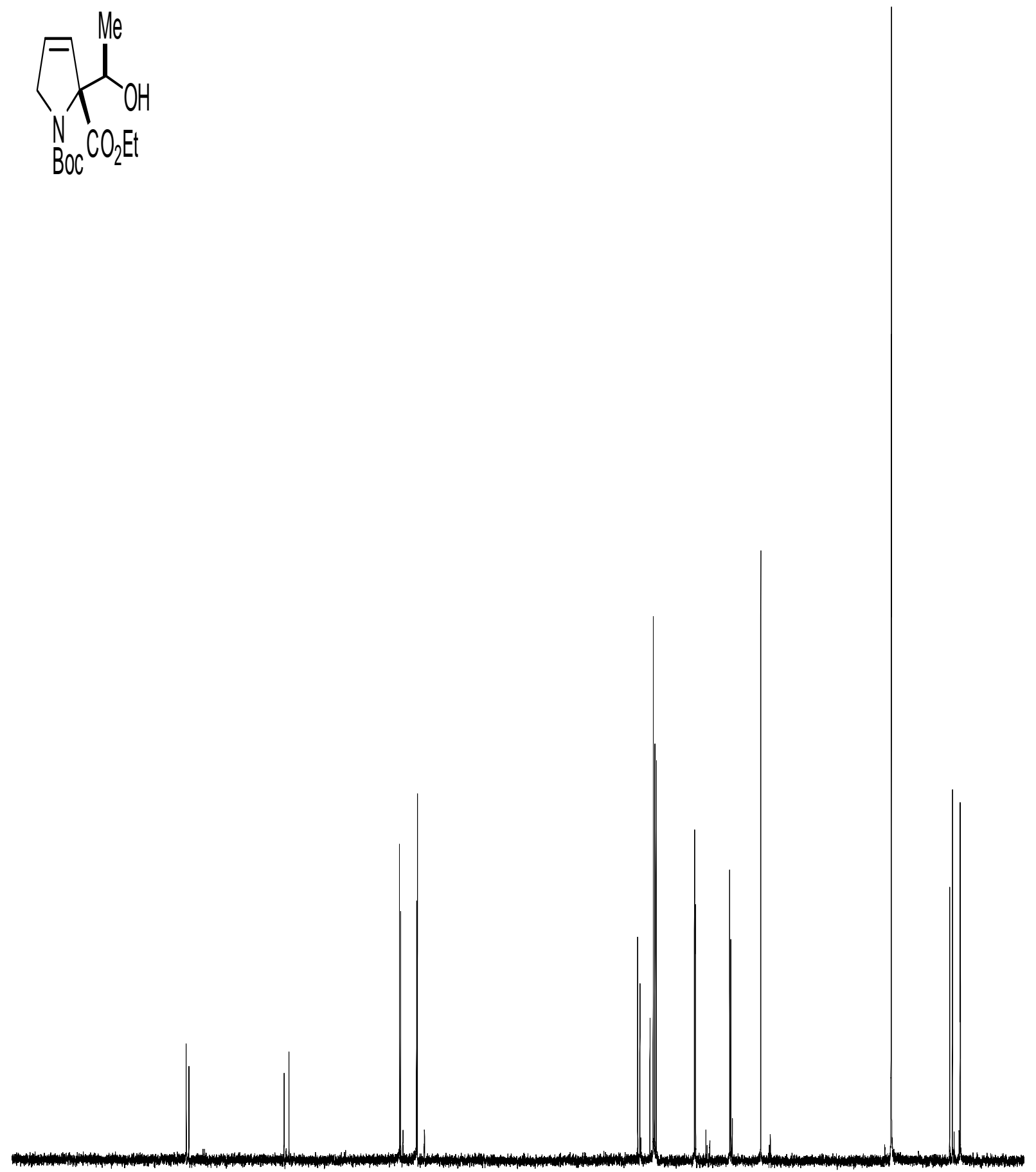

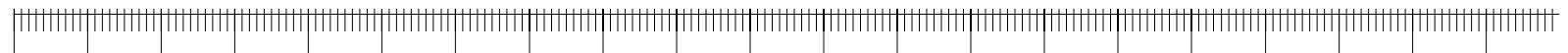

$\begin{array}{llllllllllllllllllll}200 & 190 & 180 & 170 & 160 & 150 & 140 & 130 & 120 & 110 & 100 & 90 & 80 & 70 & 60 & 50 & 40 & 30 & 20 & 10\end{array}$ chemical shift (ppm) 
${ }^{1}$ H NMR of BOM ether 21
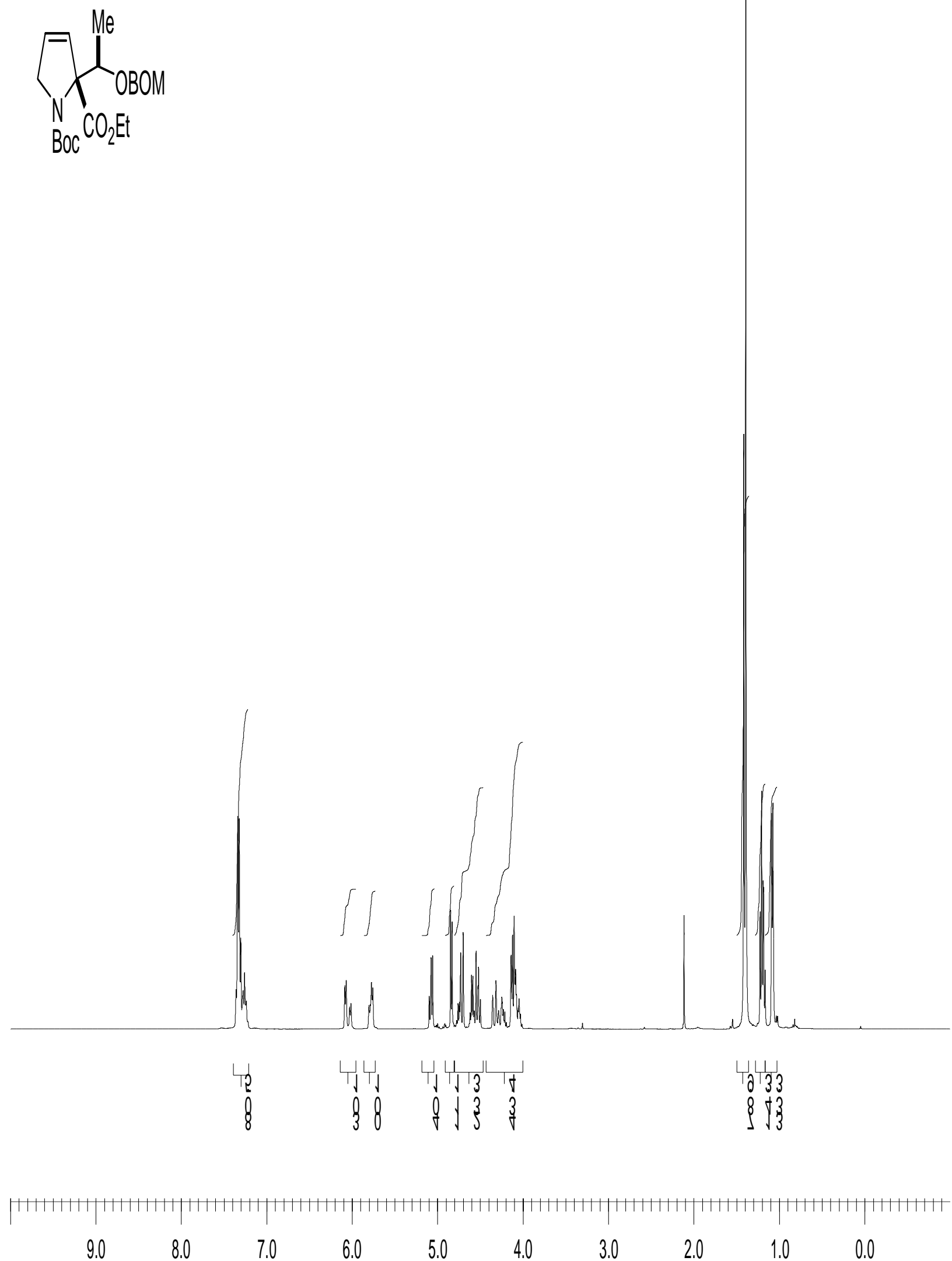
chemical shitt (ppm) 
${ }^{13} \mathrm{C}$ NMR of BOM ether 21
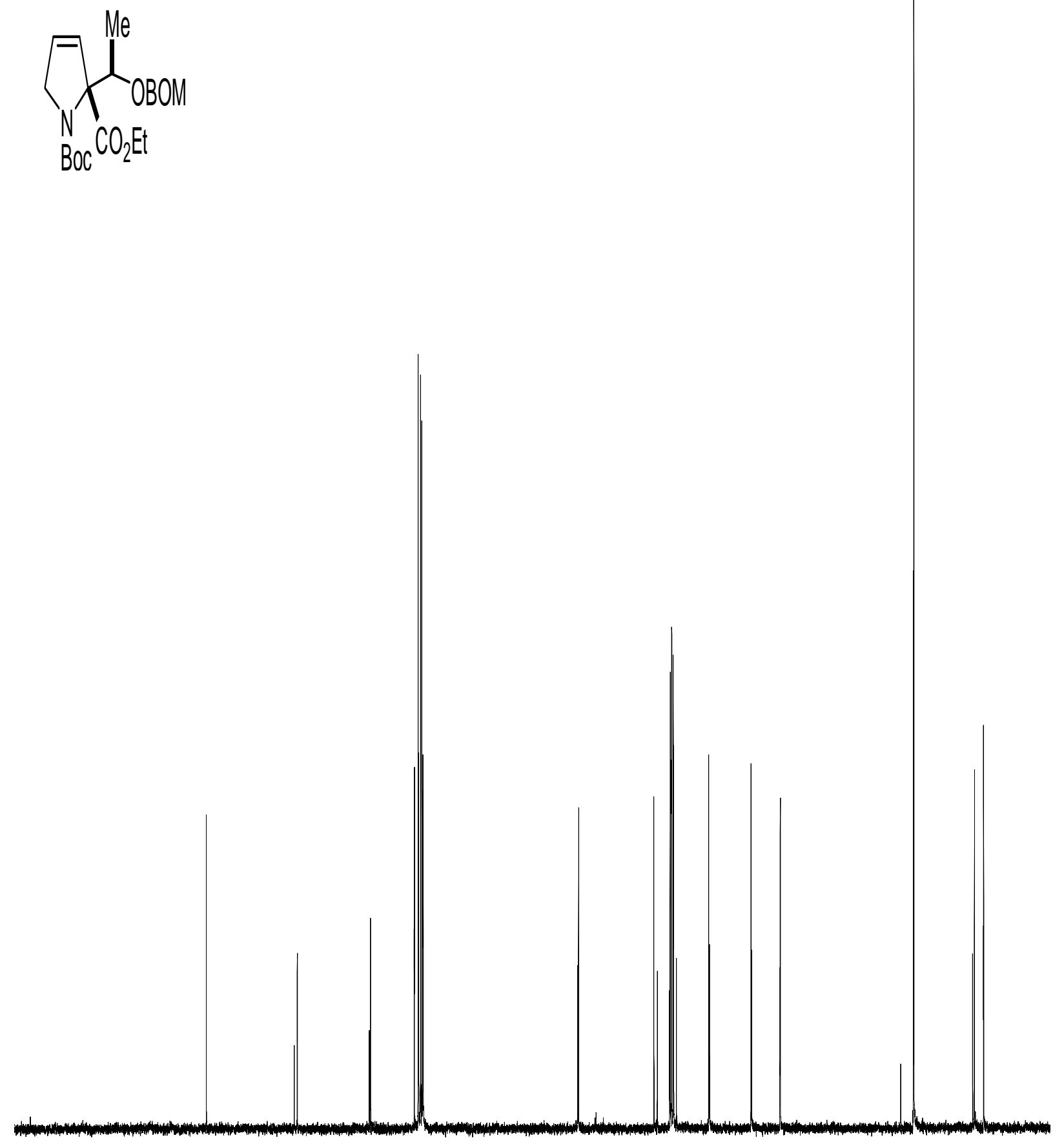

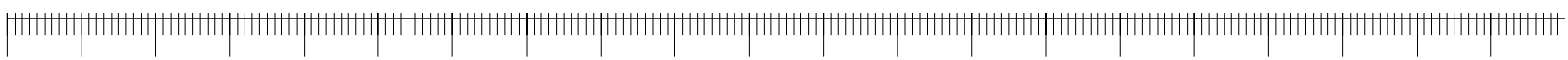

$\begin{array}{llllllllllllllllllll}200 & 190 & 180 & 170 & 160 & 150 & 140 & 130 & 120 & 110 & 100 & 90 & 80 & 70 & 60 & 50 & 40 & 30 & 20 & 10\end{array}$ chemical shitt (ppm) 
${ }^{1} \mathrm{H}$ NMR of syn-diol $\mathbf{1 1}$
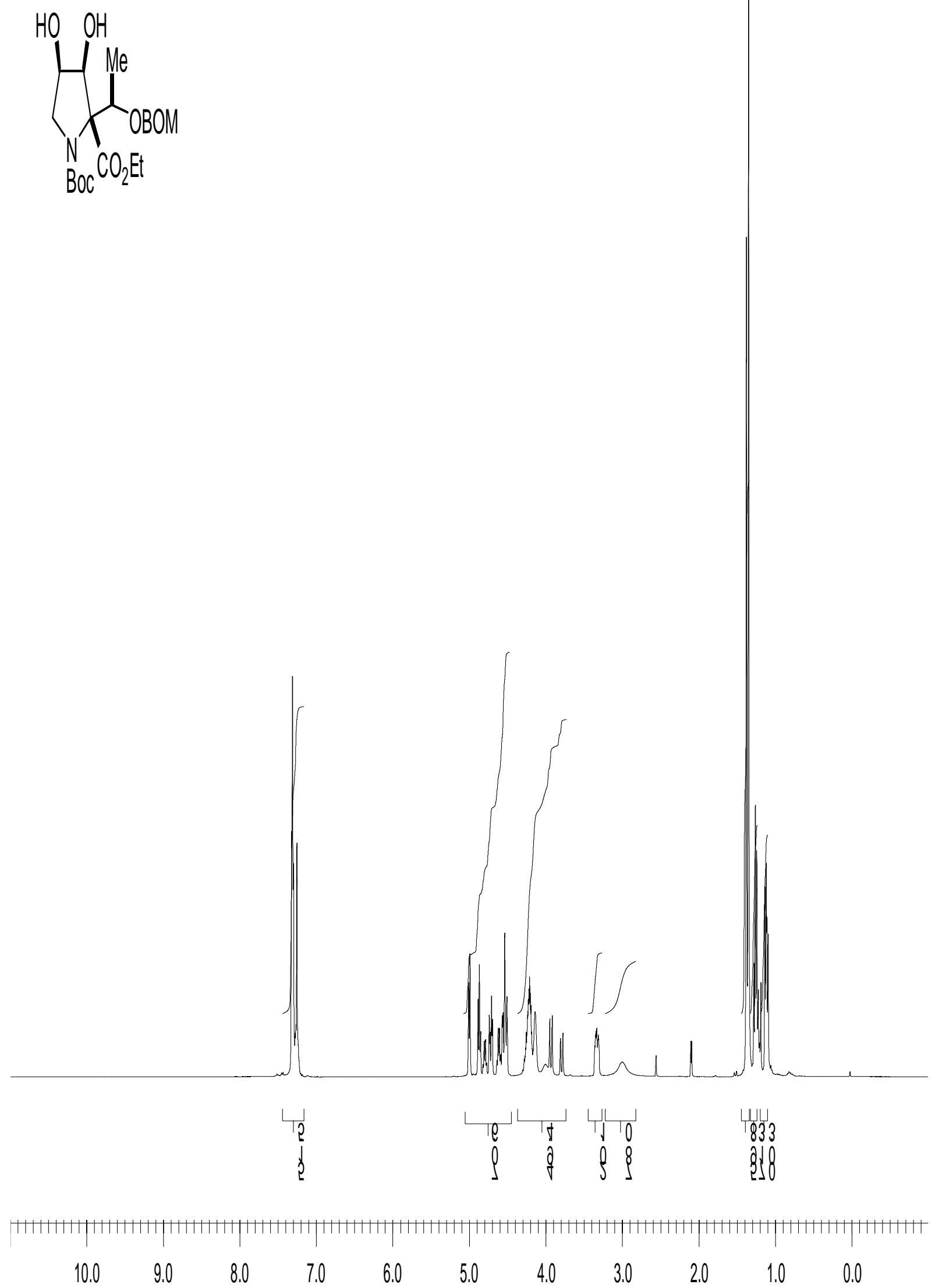

chemical shitt (opm) 
${ }^{13}$ C NMR of syn-diol 11
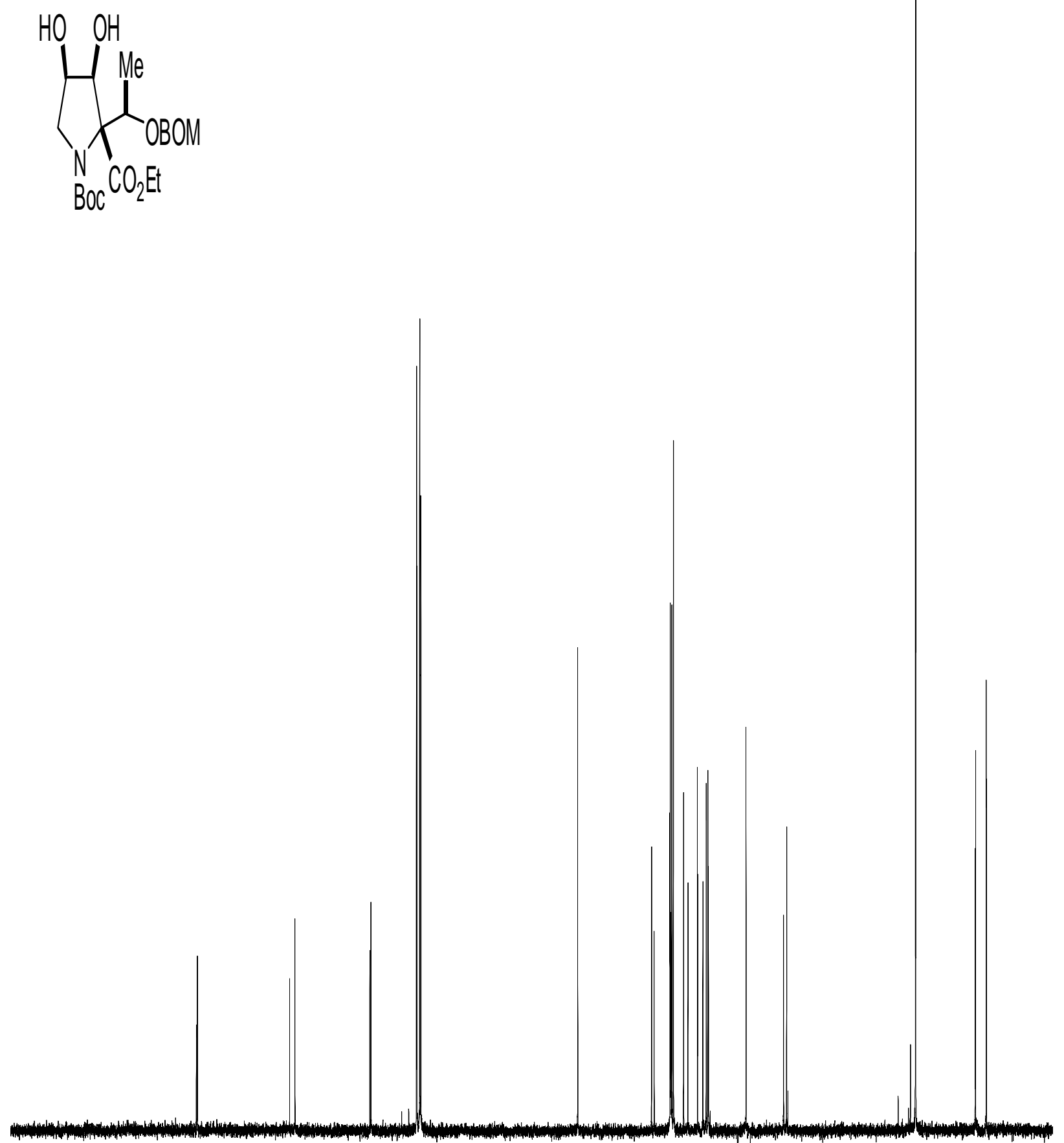

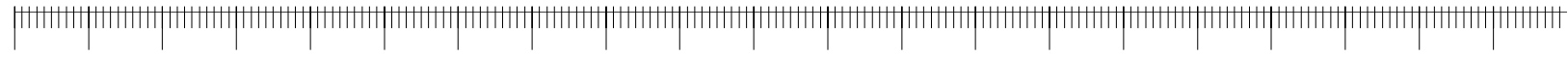

$\begin{array}{llllllllllllllllllll}200 & 190 & 180 & 170 & 160 & 150 & 140 & 130 & 120 & 110 & 100 & 90 & 80 & 70 & 60 & 50 & 40 & 30 & 20 & 10\end{array}$ chemical shitt (ppm) 
${ }^{1}$ H NMR of iodohydrin 22
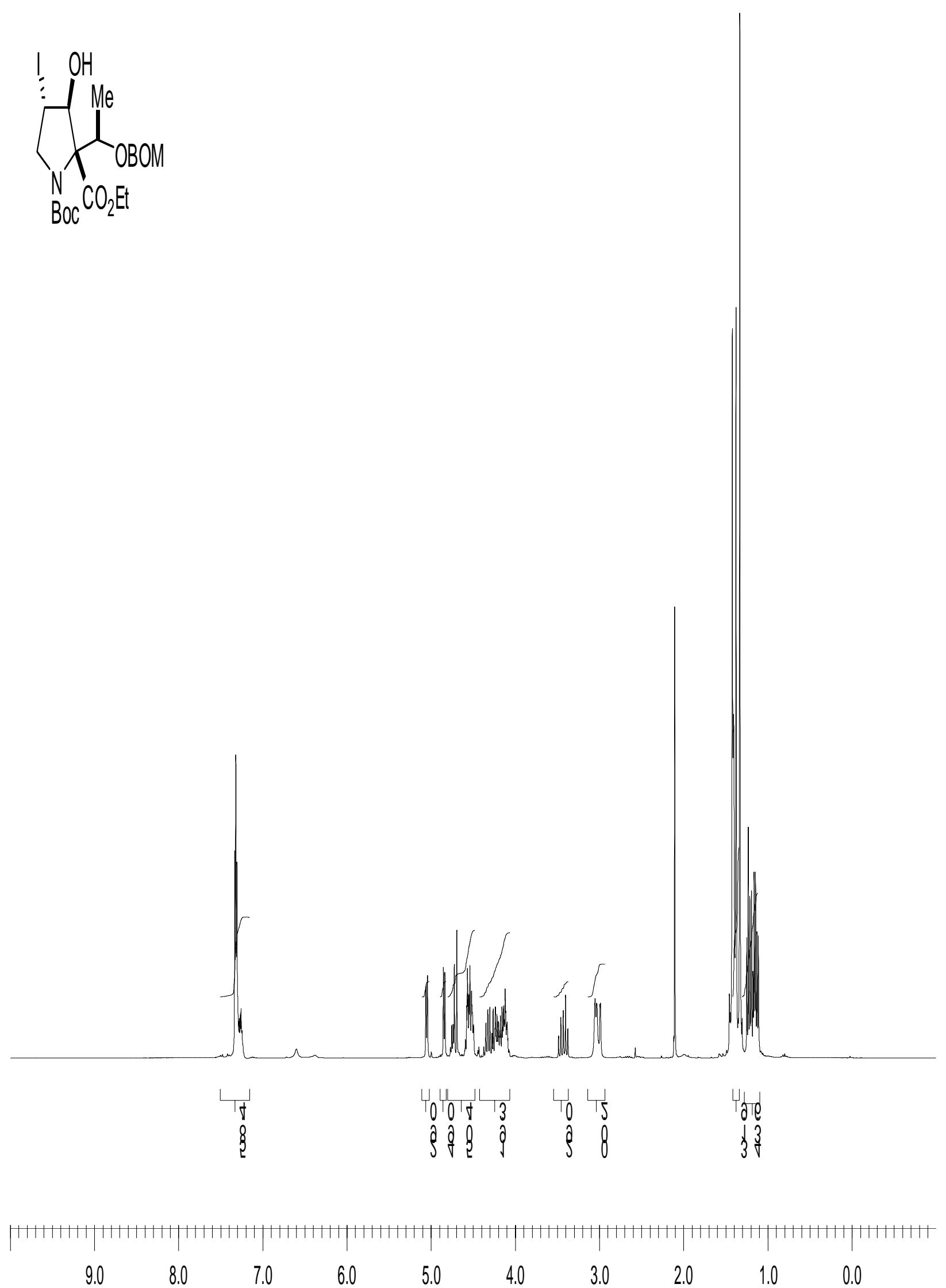
chemical shitt (ppm) 
${ }^{13} \mathrm{C}$ NMR of iodohydrin 22


$\begin{array}{llllllllllllllllllll}200 & 190 & 180 & 170 & 160 & 150 & 140 & 130 & 120 & 110 & 100 & 90 & 80 & 70 & 60 & 50 & 40 & 30 & 20 & 10\end{array}$ chemical shitt (ppm) 
${ }^{1} \mathrm{H}$ NMR of hydroxyester $\mathbf{1 2}$
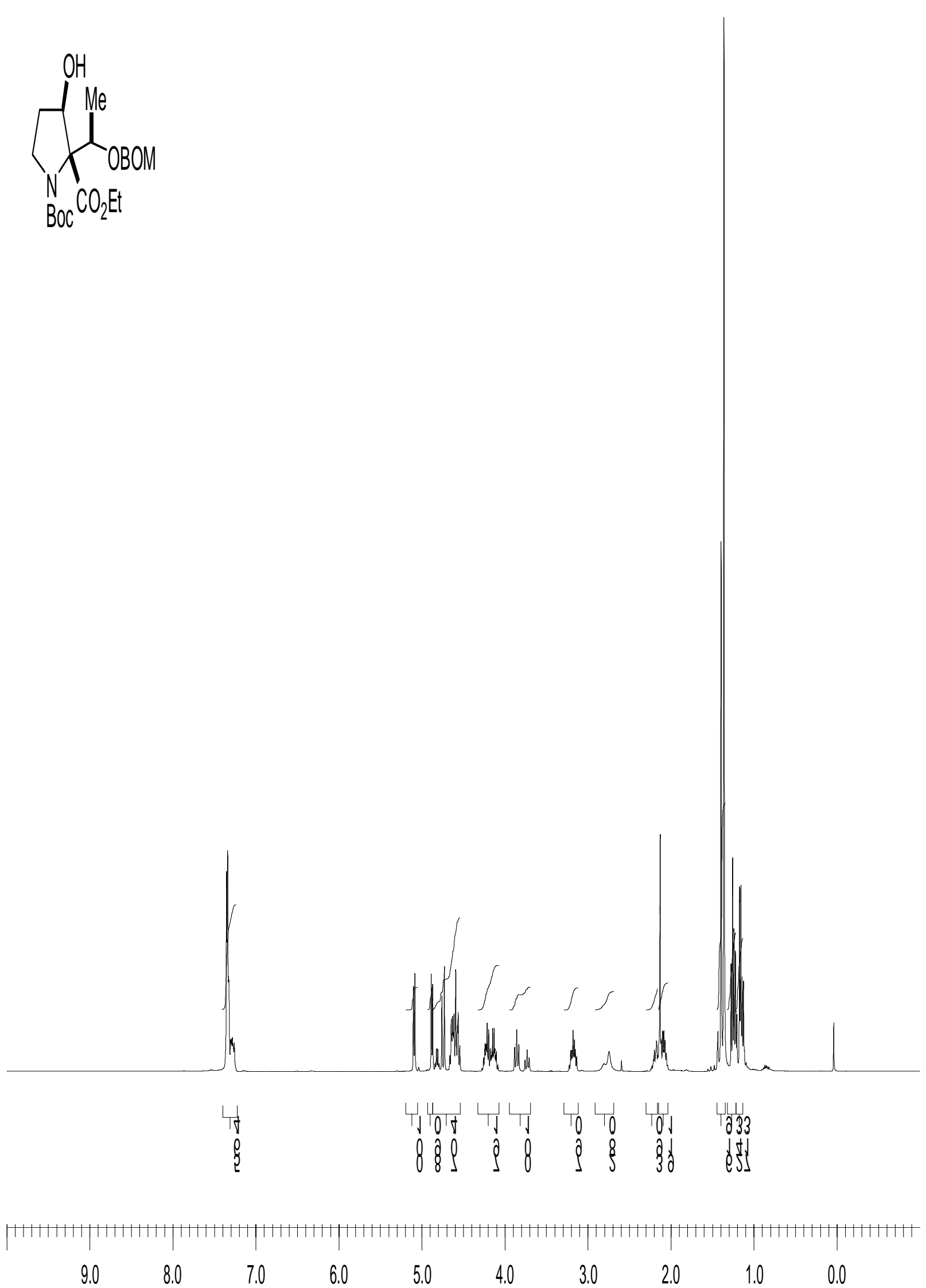
chemical shift (ppm) 


\section{${ }^{13} \mathrm{C}$ NMR of hydroxyester $\mathbf{1 2}$}
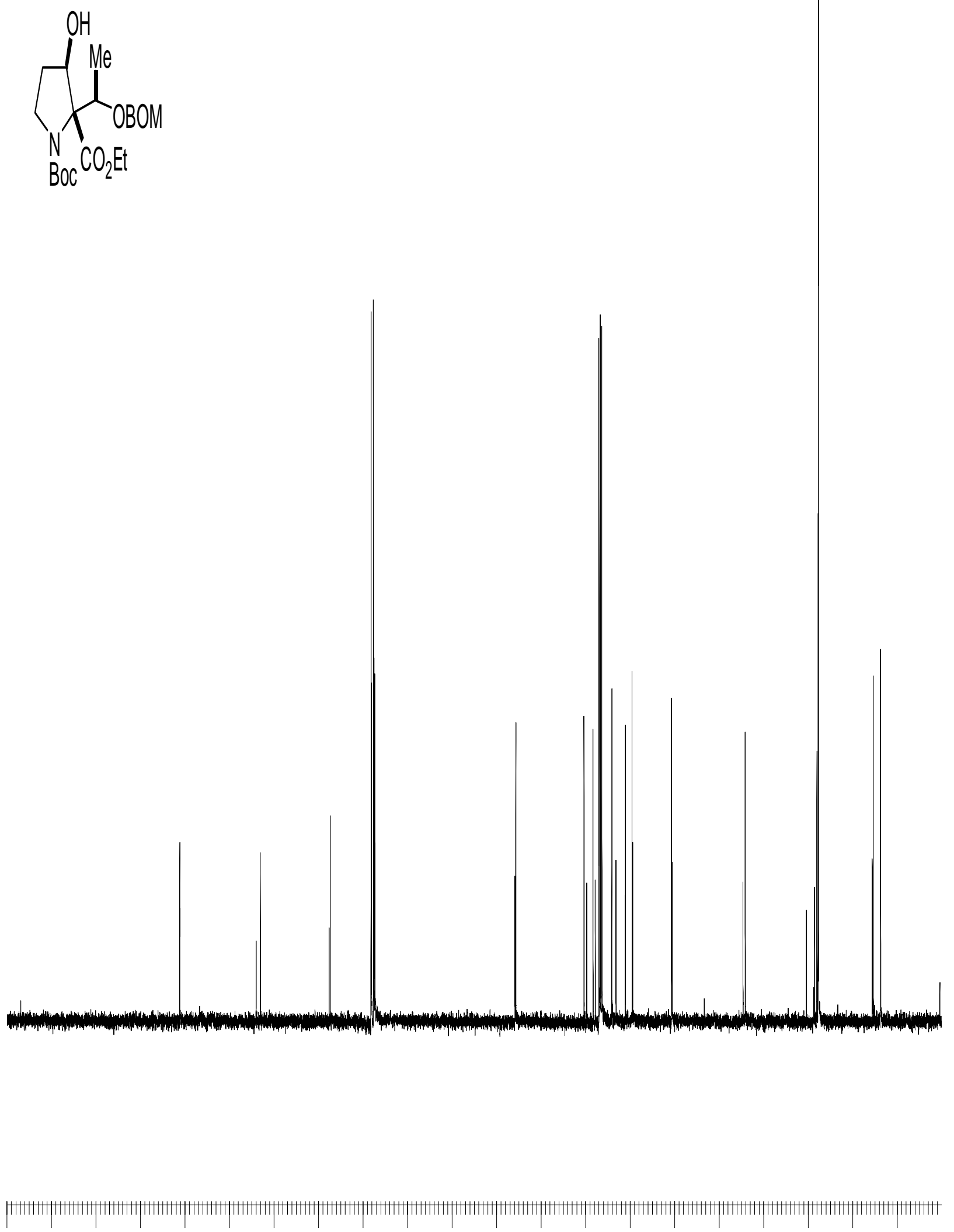

$\begin{array}{llllllllllllllllllll}200 & 190 & 180 & 170 & 160 & 150 & 140 & 130 & 120 & 110 & 100 & 90 & 80 & 70 & 60 & 50 & 40 & 30 & 20 & 10\end{array}$ chemical shift (ppm) 
${ }^{1} \mathrm{H}$ NMR of ketoester $\mathbf{1 3}$
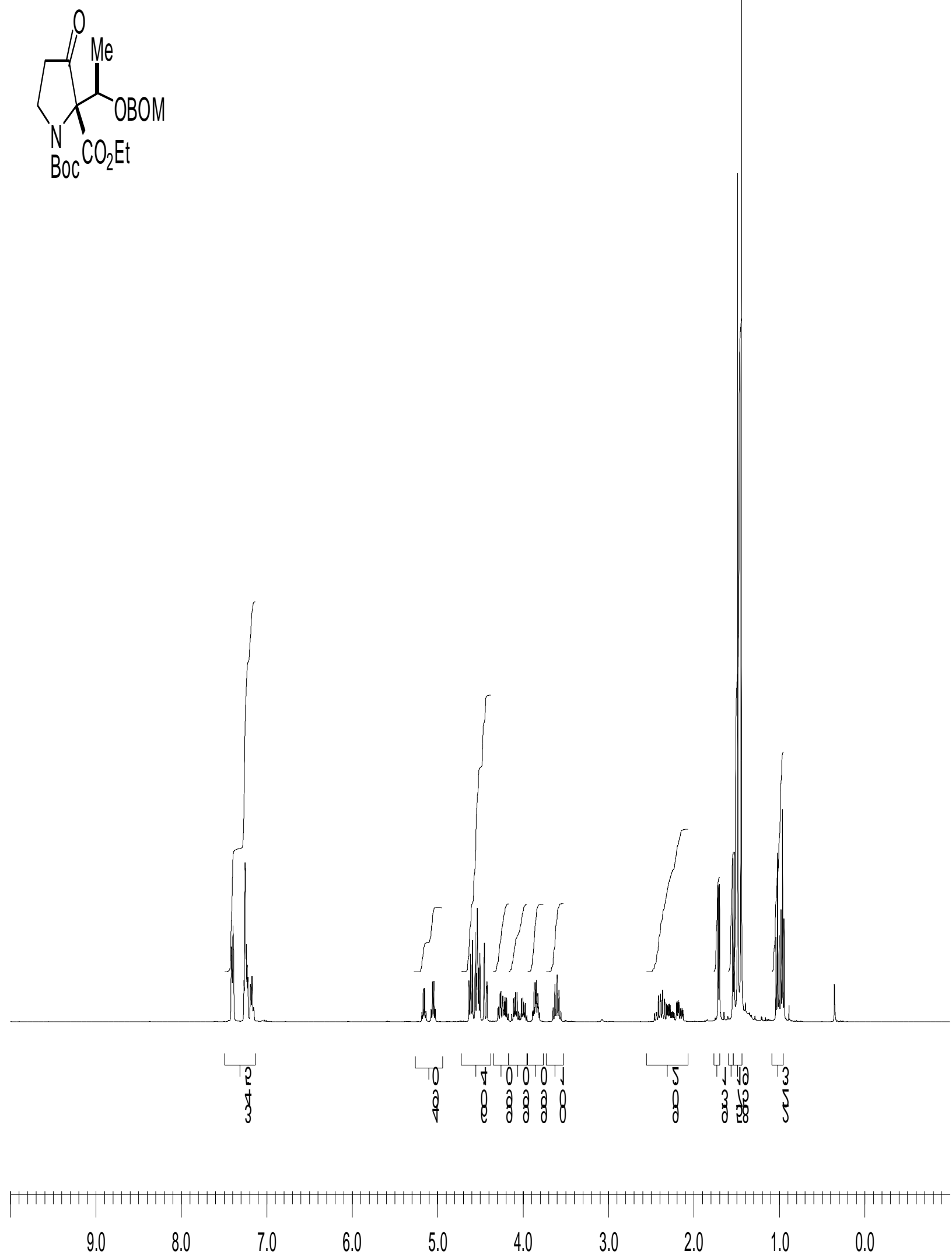

chemical shift (ppm) 


\section{${ }^{13} \mathrm{C}$ NMR of ketoester $\mathbf{1 3}$}
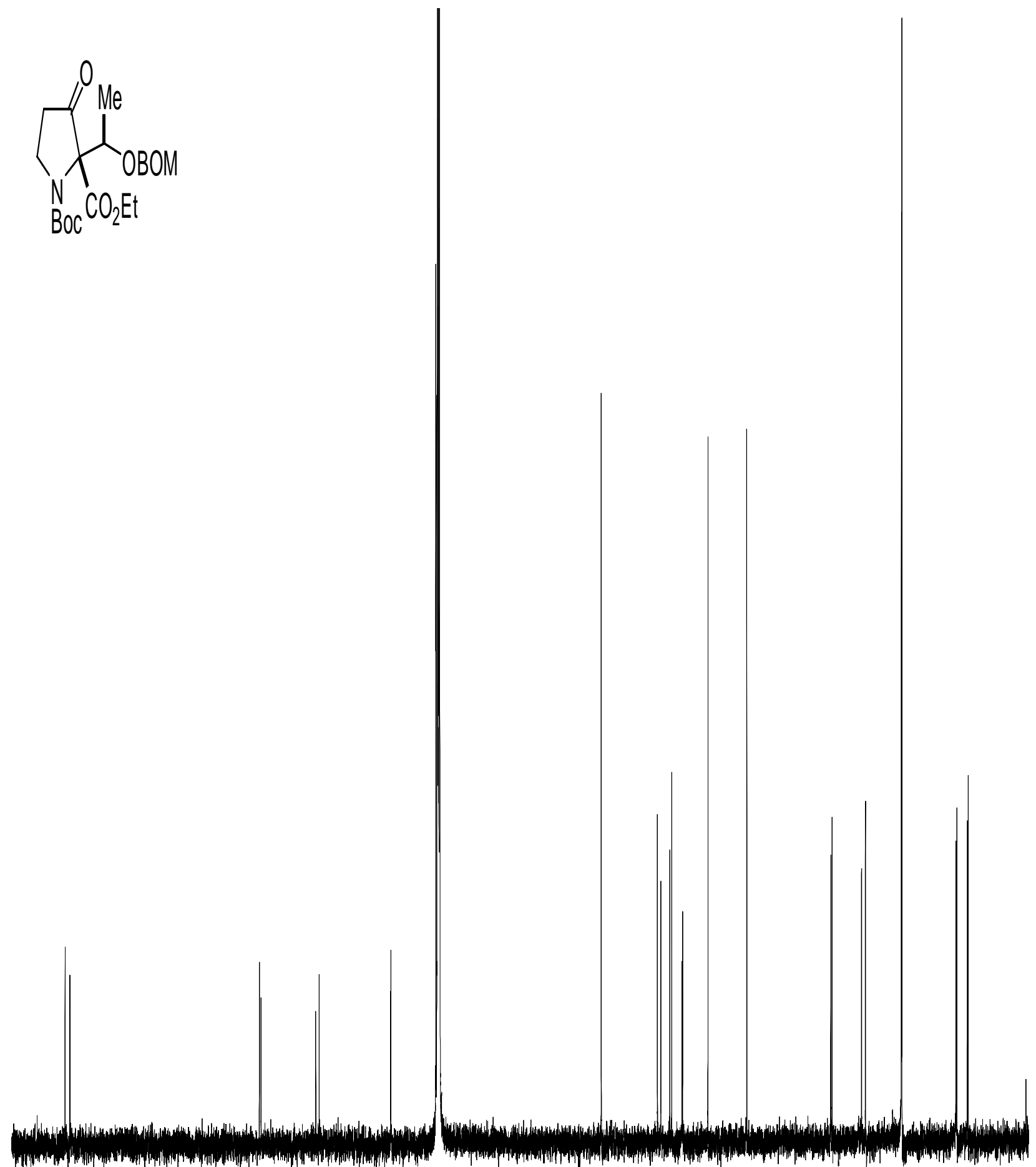

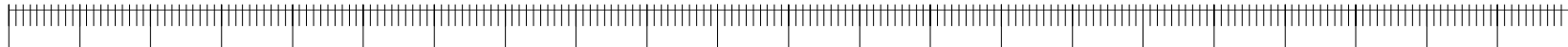

$\begin{array}{lllllllllllllllllllll}210 & 200 & 190 & 180 & 170 & 160 & 150 & 140 & 130 & 120 & 110 & 100 & 90 & 80 & 70 & 60 & 50 & 40 & 30 & 20 & 10\end{array}$ chemical shitt (ppm) 
${ }^{1} \mathrm{H}$ NMR of methylated ketoester 14
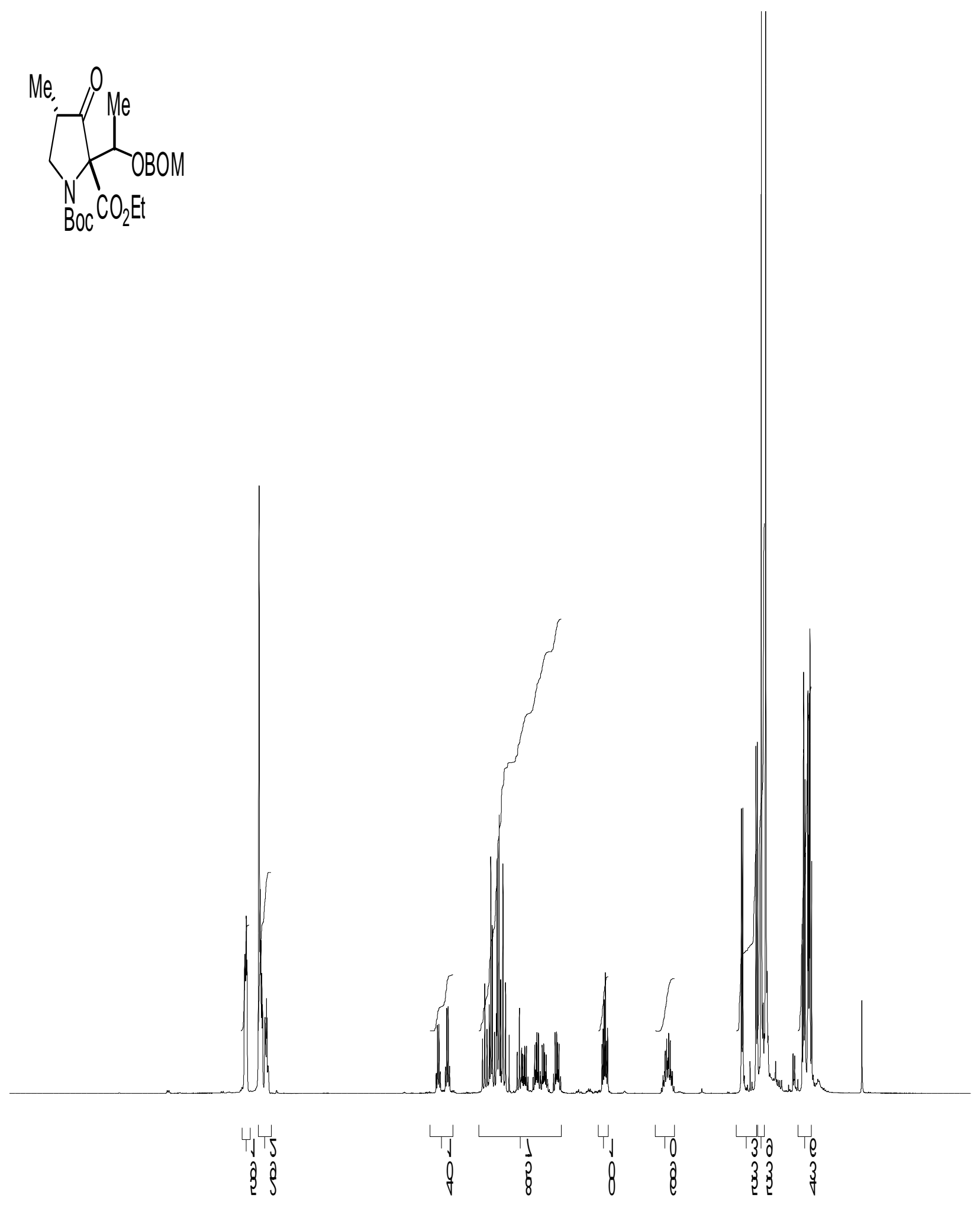
${ }^{13} \mathrm{C}$ NMR of methylated ketoester 14
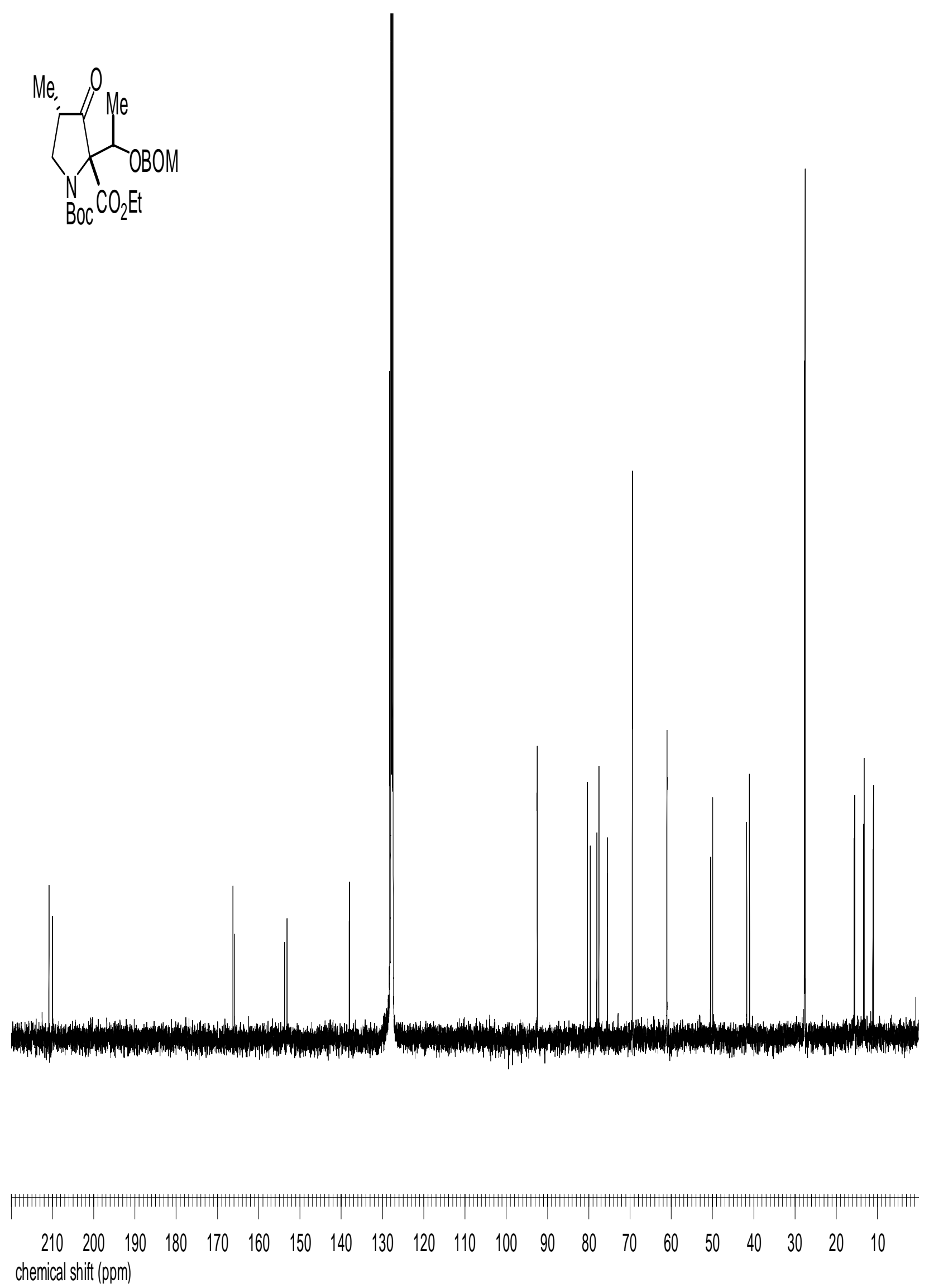
${ }^{1} \mathrm{H}$ NMR of homoallyl alcohol $\mathbf{1 5}$
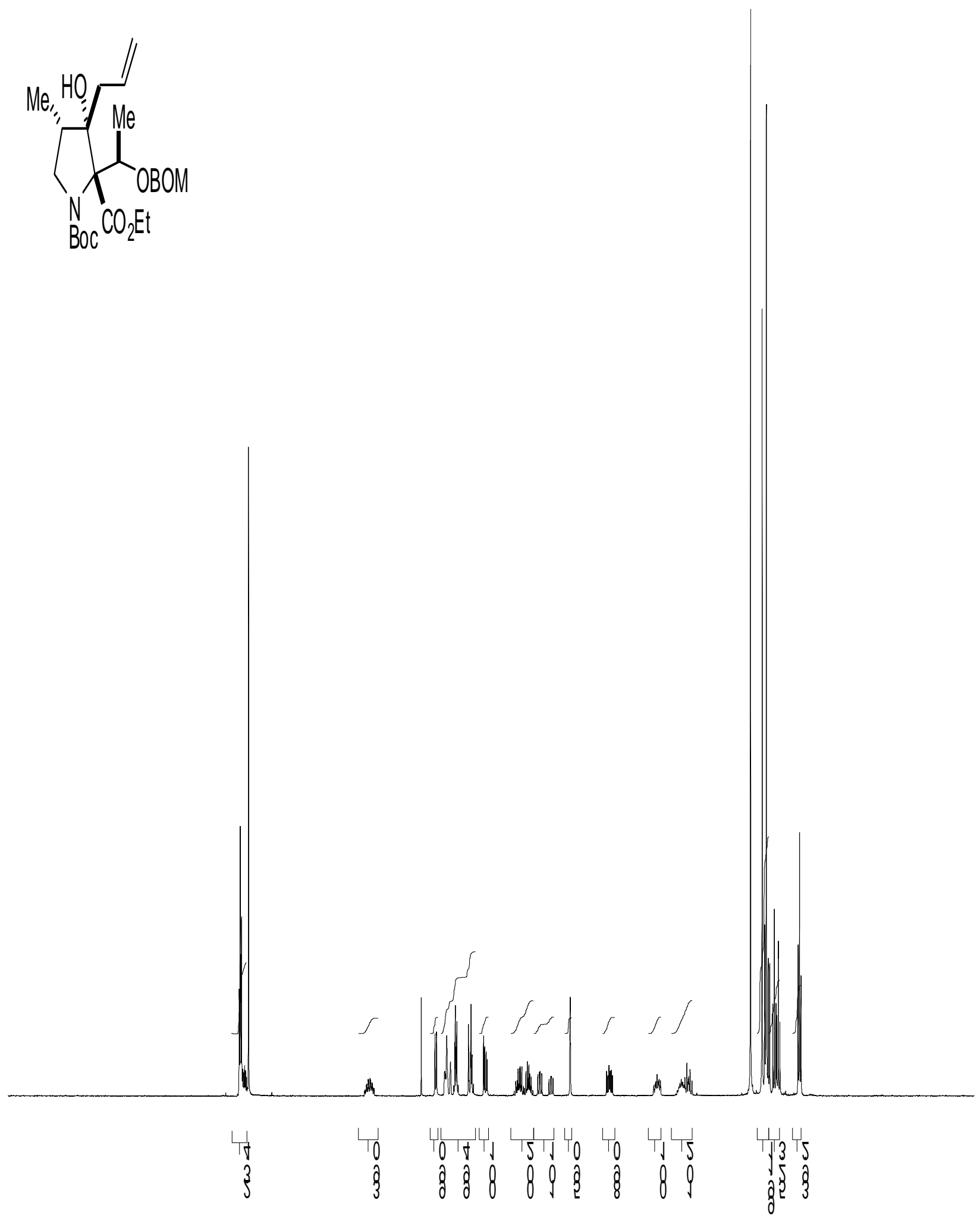

\begin{tabular}{|c|c|c|c|c|c|c|c|c|c|}
\hline $\begin{array}{c}9.0 \\
\text { chemical shitt (ppm) }\end{array}$ & 8.0 & 7.0 & 6.0 & 5.0 & 4.0 & 3.0 & 2.0 & 1.0 & 0.0 \\
\hline
\end{tabular}


${ }^{13} \mathrm{C}$ NMR of homoallyl alcohol $\mathbf{1 5}$
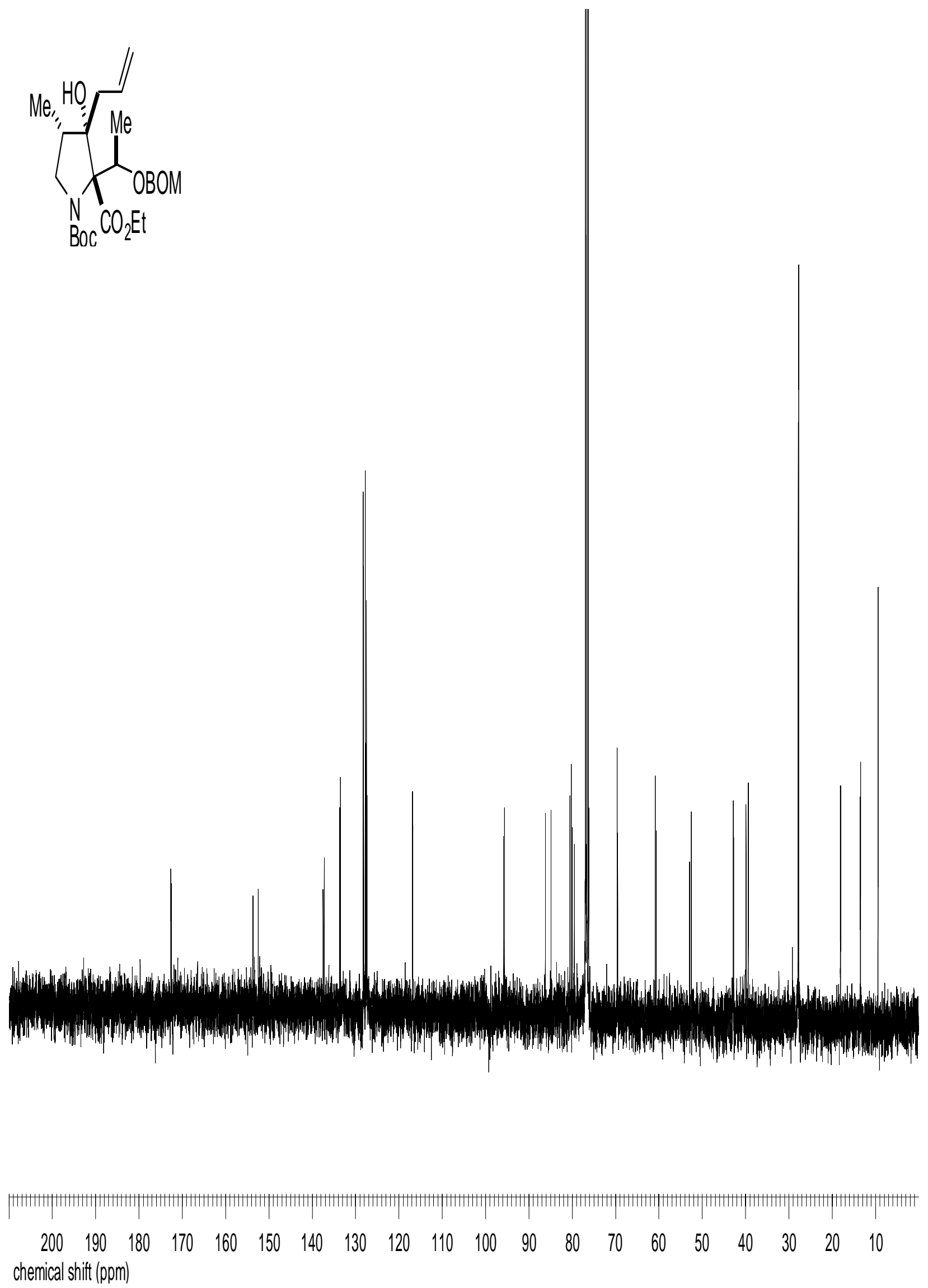


\section{${ }^{1} \mathrm{H}$ NMR of allyl alcohol $\mathbf{1 6}$}

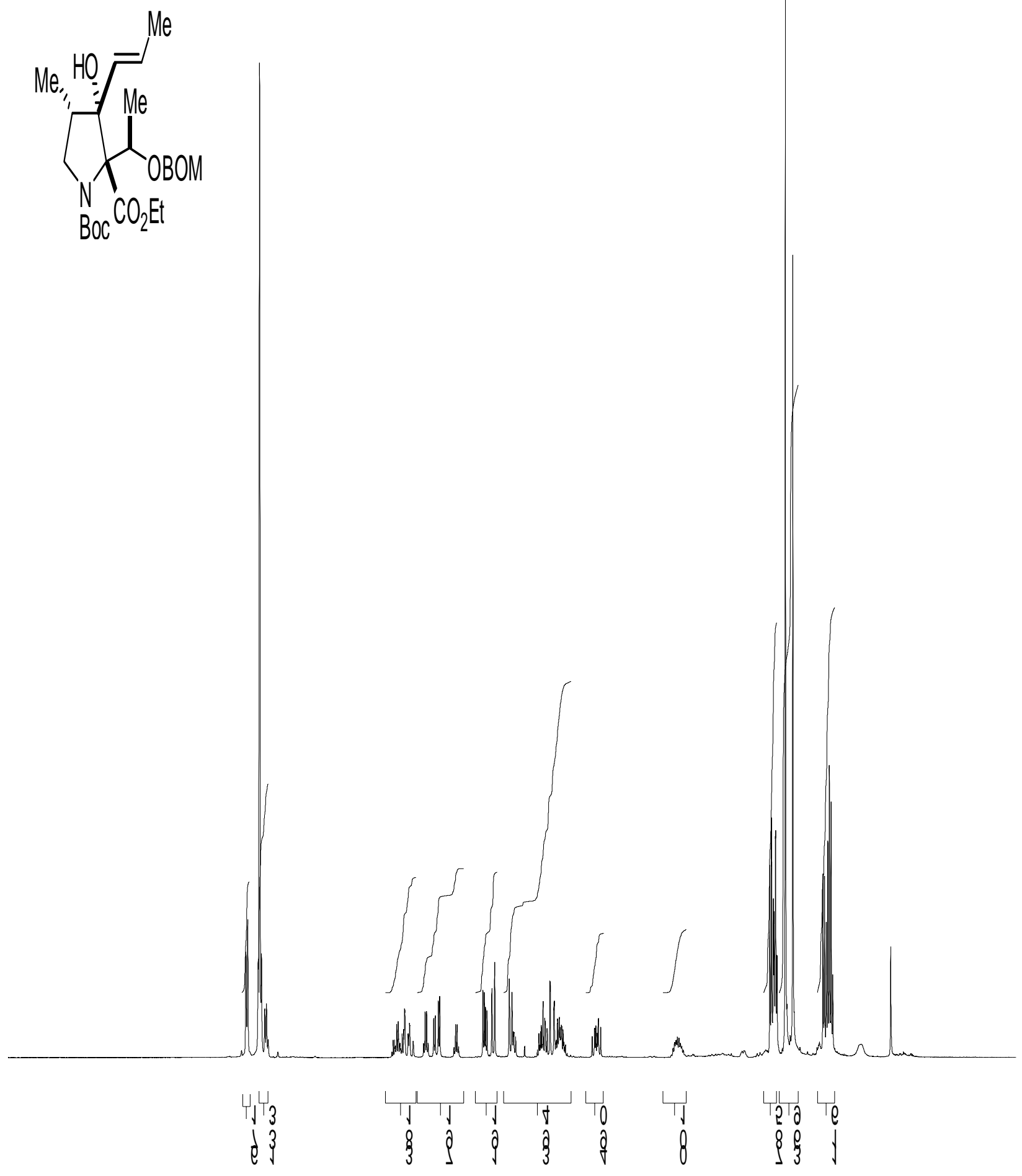

\begin{tabular}{|c|c|c|c|c|c|c|c|c|c|}
\hline $\begin{array}{c}9.0 \\
\text { chemical shift (ppm) }\end{array}$ & 8.0 & 7.0 & 6.0 & 5.0 & 4.0 & 3.0 & 2.0 & 1.0 & 0.0 \\
\hline
\end{tabular}


${ }^{13} \mathrm{C}$ NMR of allyl alcohol 16
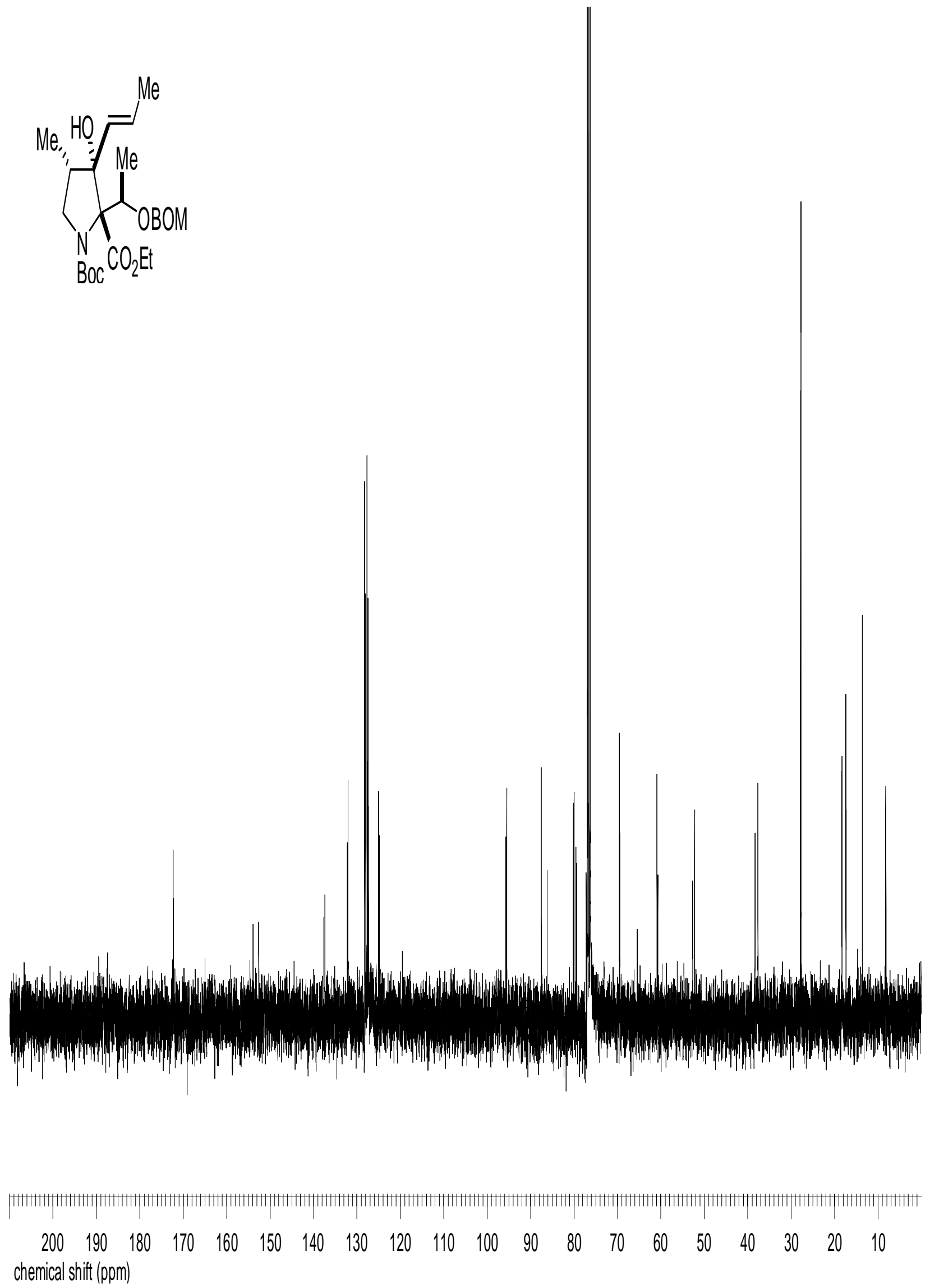
${ }^{1}$ H NMR of 1,2-diol 23

chemical shift (ppm) 
${ }^{13} \mathrm{C}$ NMR of 1,2-diol 23
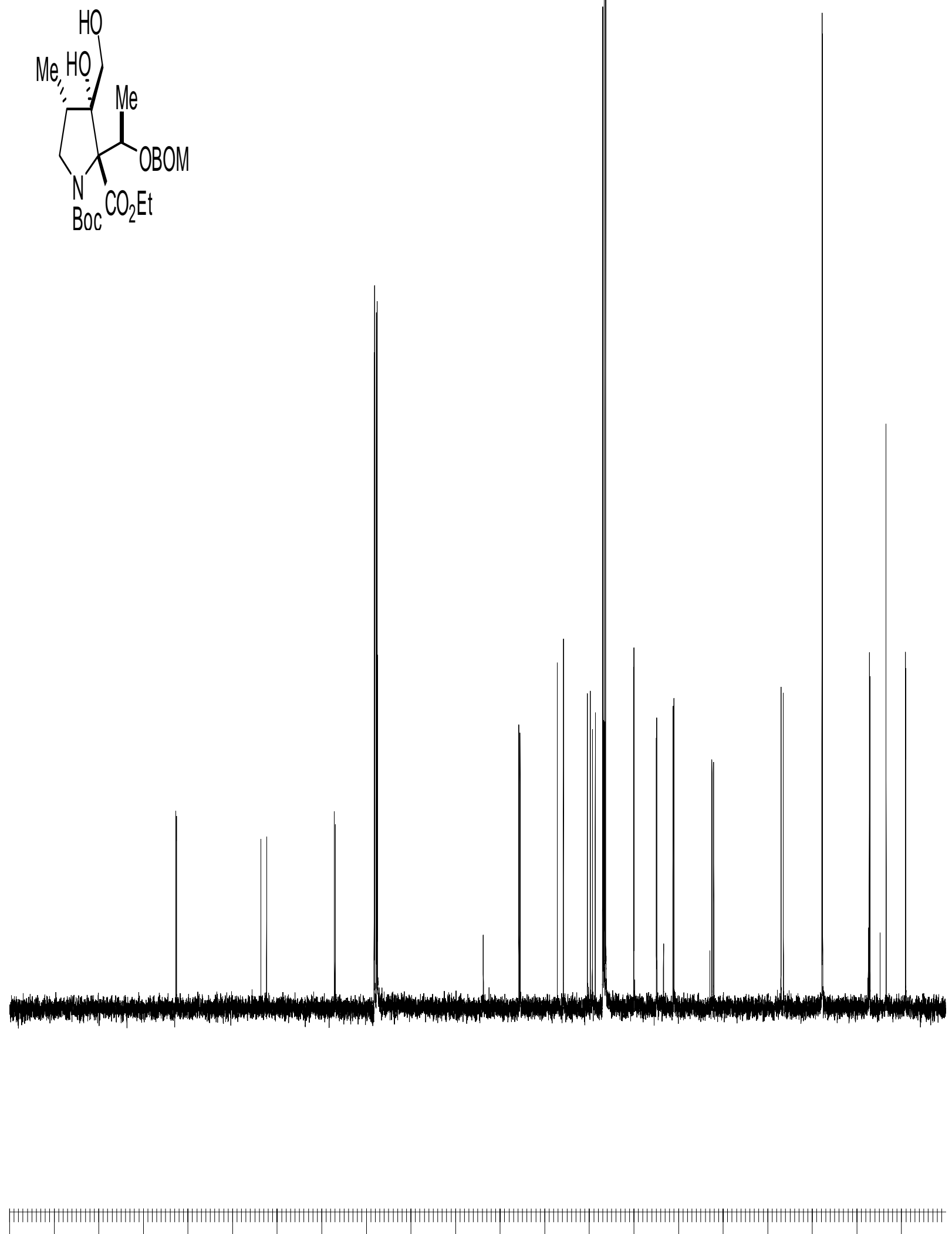

$\begin{array}{llllllllllllllllllll}200 & 190 & 180 & 170 & 160 & 150 & 140 & 130 & 120 & 110 & 100 & 90 & 80 & 70 & 60 & 50 & 40 & 30 & 20 & 10\end{array}$ chemical shift (ppm) 
${ }^{1} \mathrm{H}$ NMR of acetonide $\mathbf{1 7}$
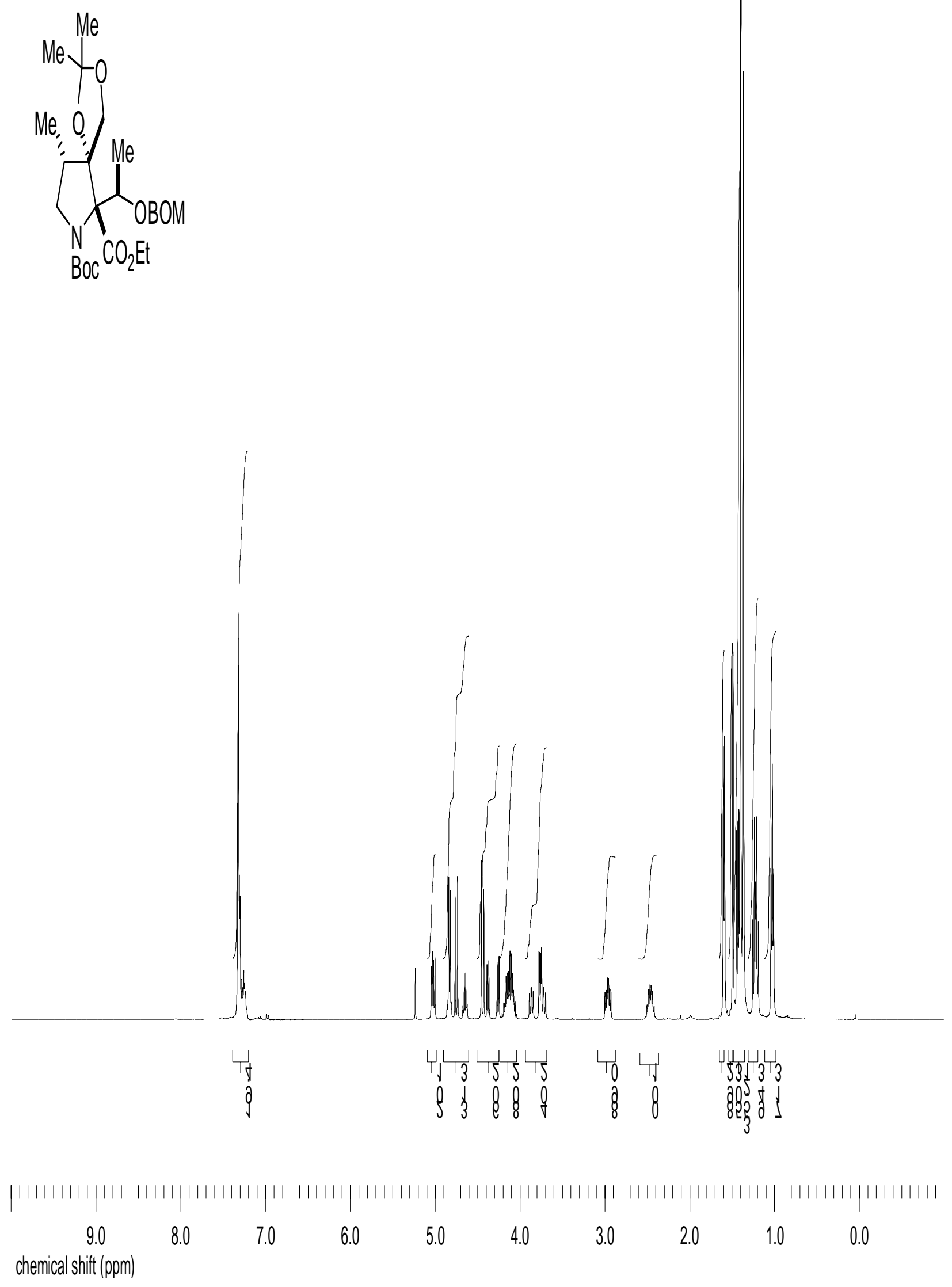
${ }^{13} \mathrm{C}$ NMR of acetonide $\mathbf{1 7}$
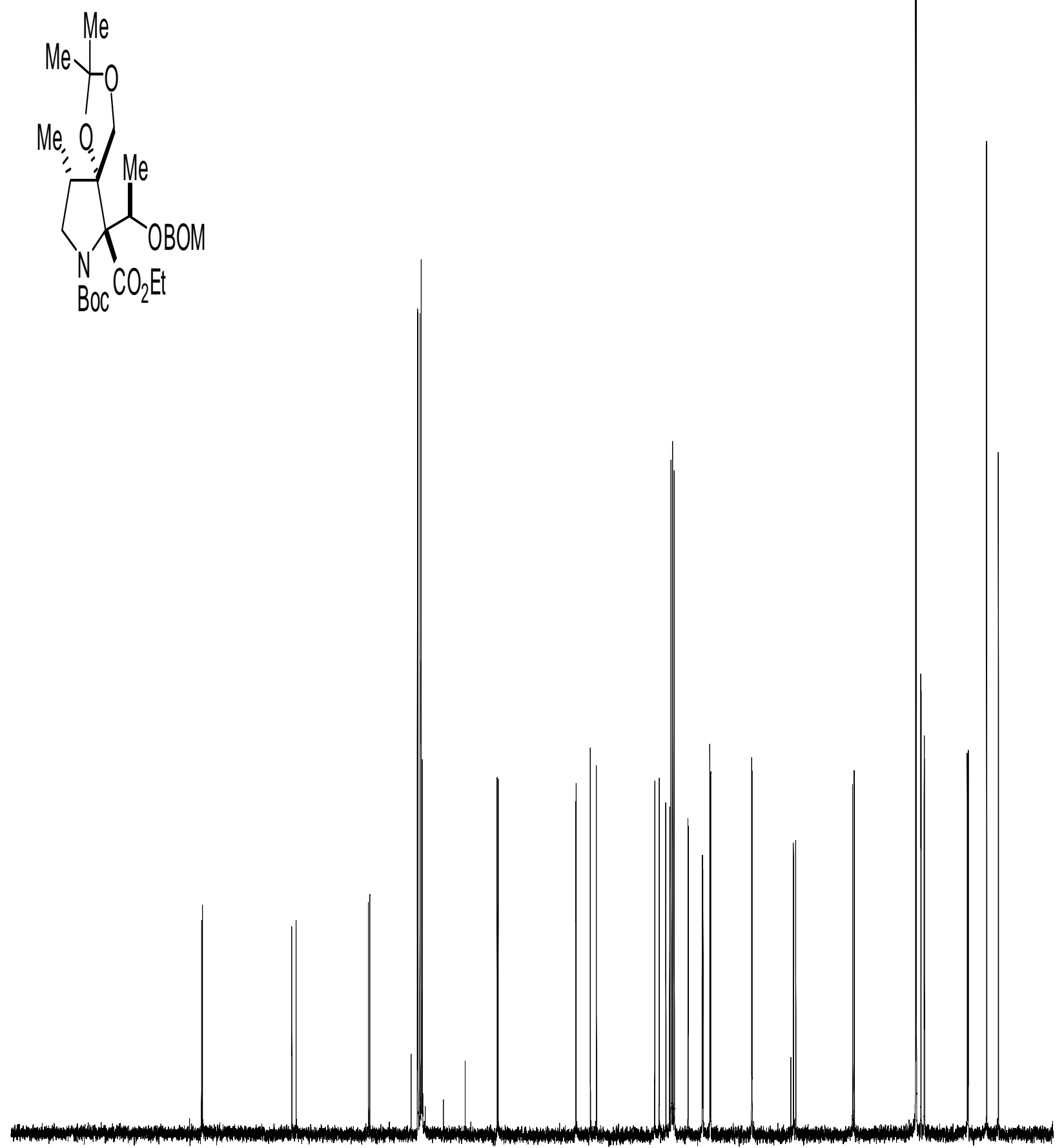
chemical shitt (ppm) 


\section{${ }^{1} \mathrm{H}$ NMR of $N$-Boc lactam 18}
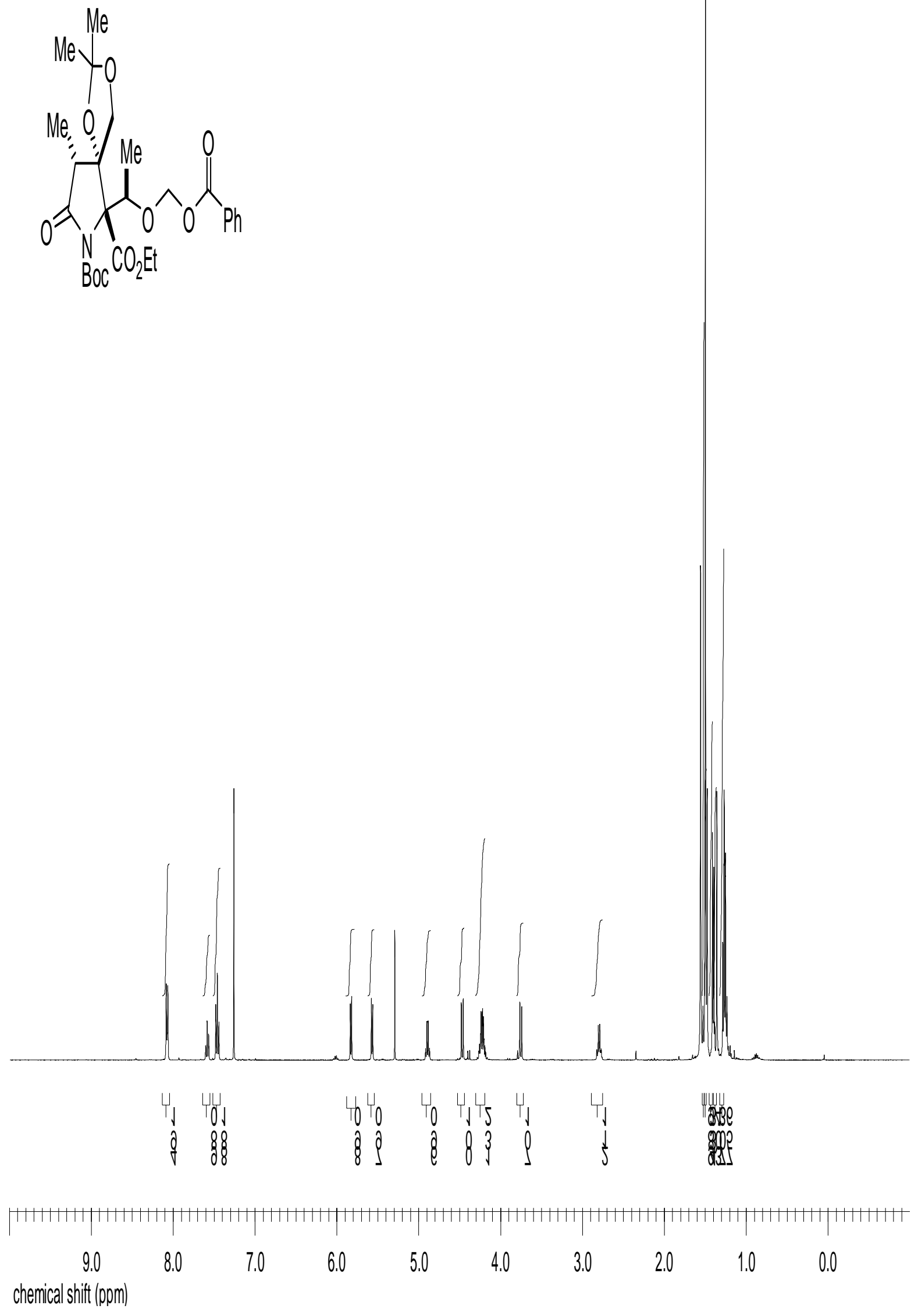
${ }^{13} \mathrm{C}$ NMR of $N$-Boc lactam 18
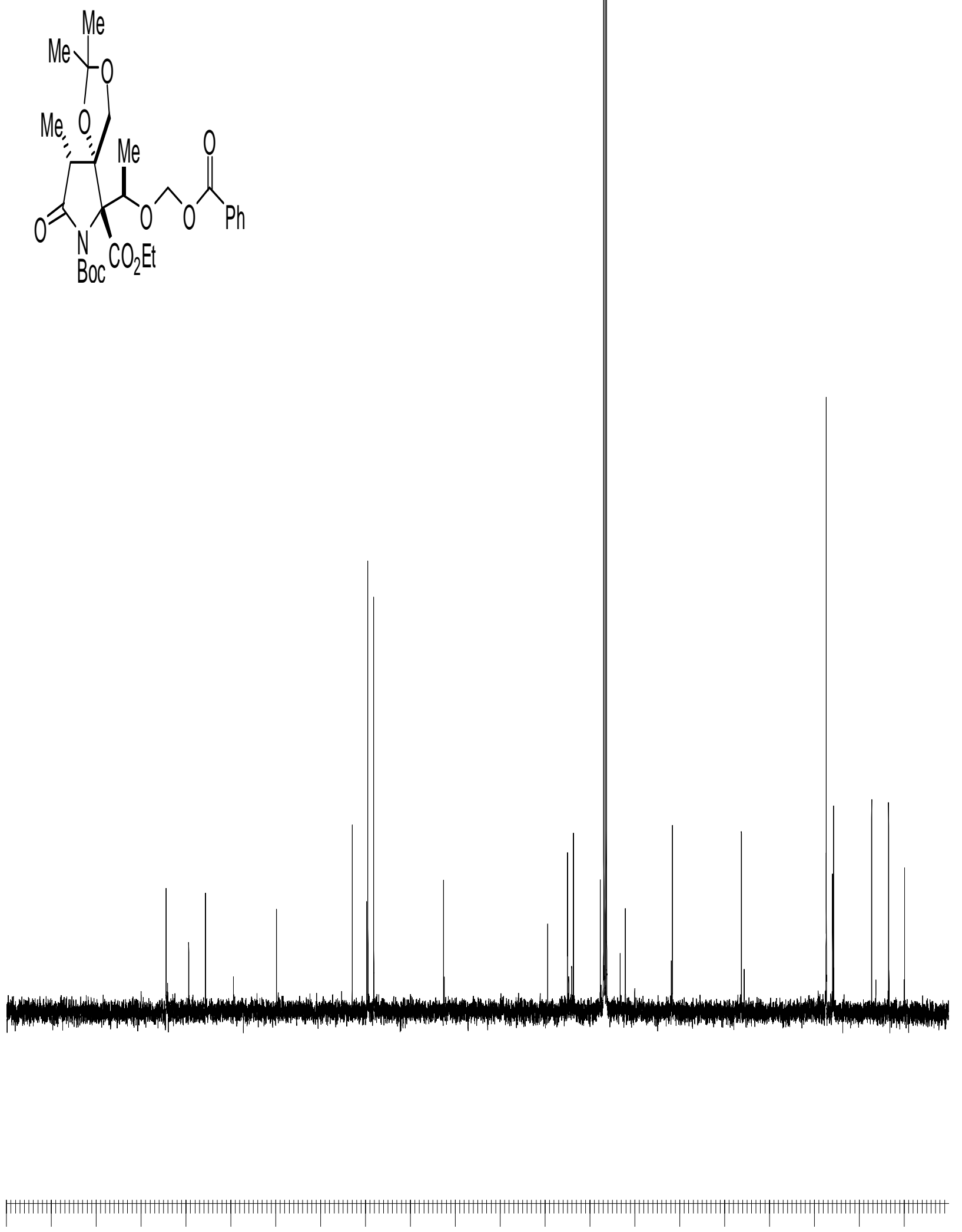

$\begin{array}{llllllllllllllllllll}200 & 190 & 180 & 170 & 160 & 150 & 140 & 130 & 120 & 110 & 100 & 90 & 80 & 70 & 60 & 50 & 40 & 30 & 20 & 10\end{array}$ chemical shift (ppm) 


\section{${ }^{1}$ H NMR of lactam 24}
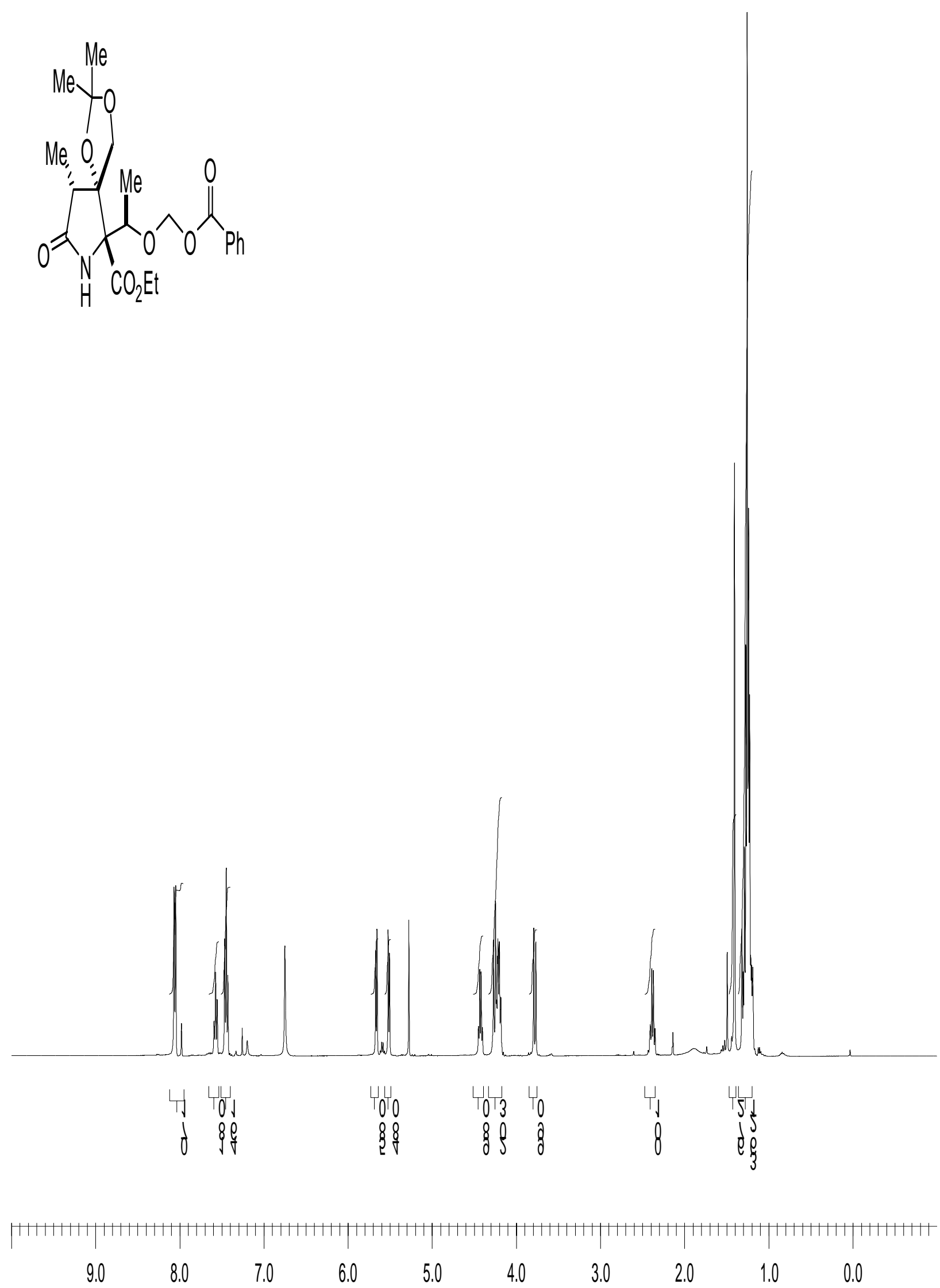

chemical shift (ppm) 
${ }^{13} \mathrm{C}$ NMR of lactam 24
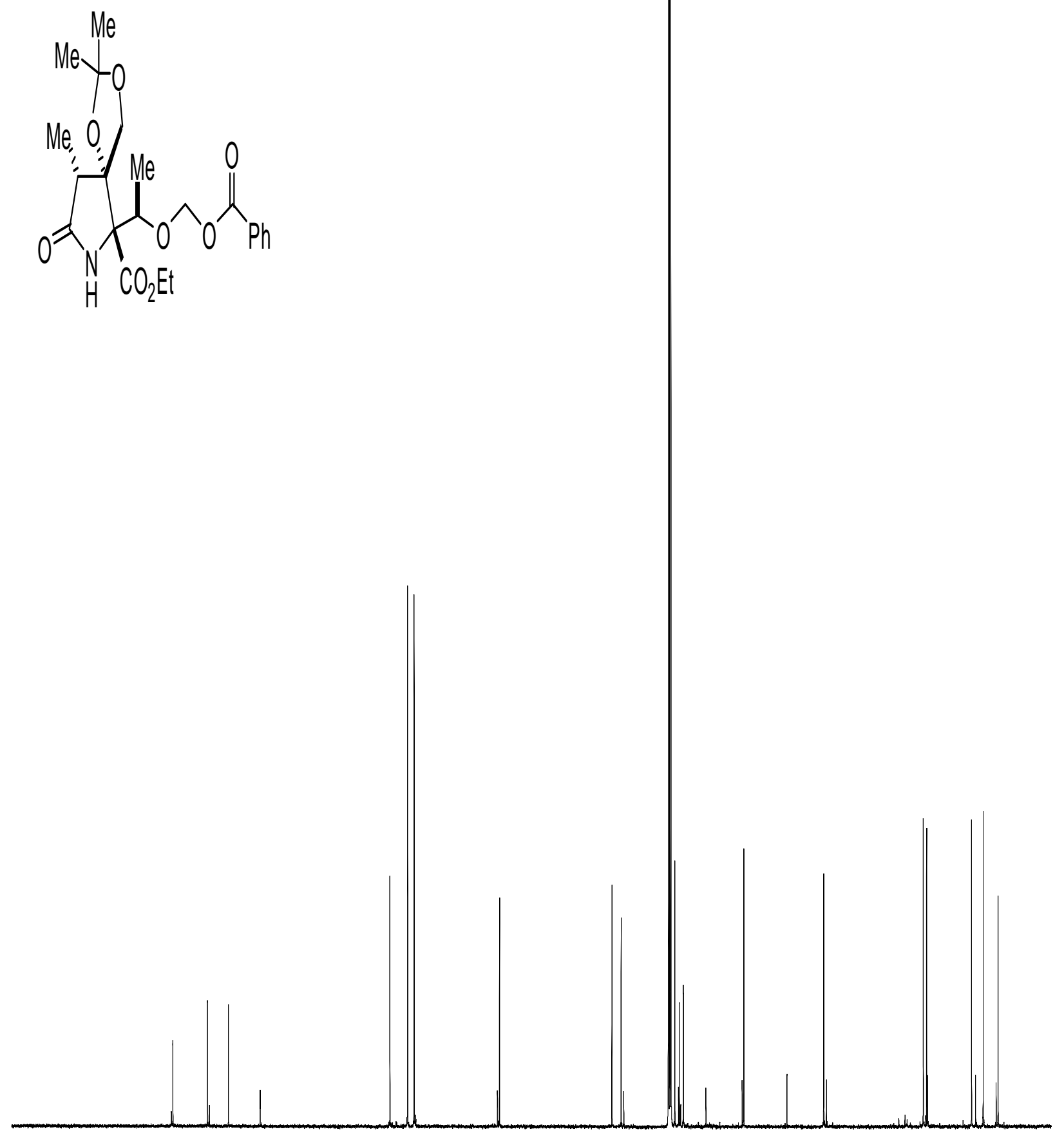

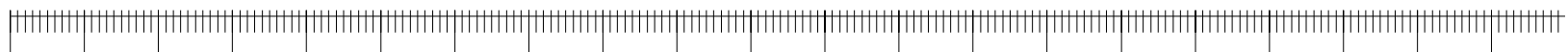
$\begin{array}{llllllllllllllllllll}200 & 190 & 180 & 170 & 160 & 150 & 140 & 130 & 120 & 110 & 100 & 90 & 80 & 70 & 60 & 50 & 40 & 30 & 20 & 10\end{array}$ chemical shitt (ppm) 
${ }^{1} \mathrm{H}$ NMR of $N$-methylated lactam 19
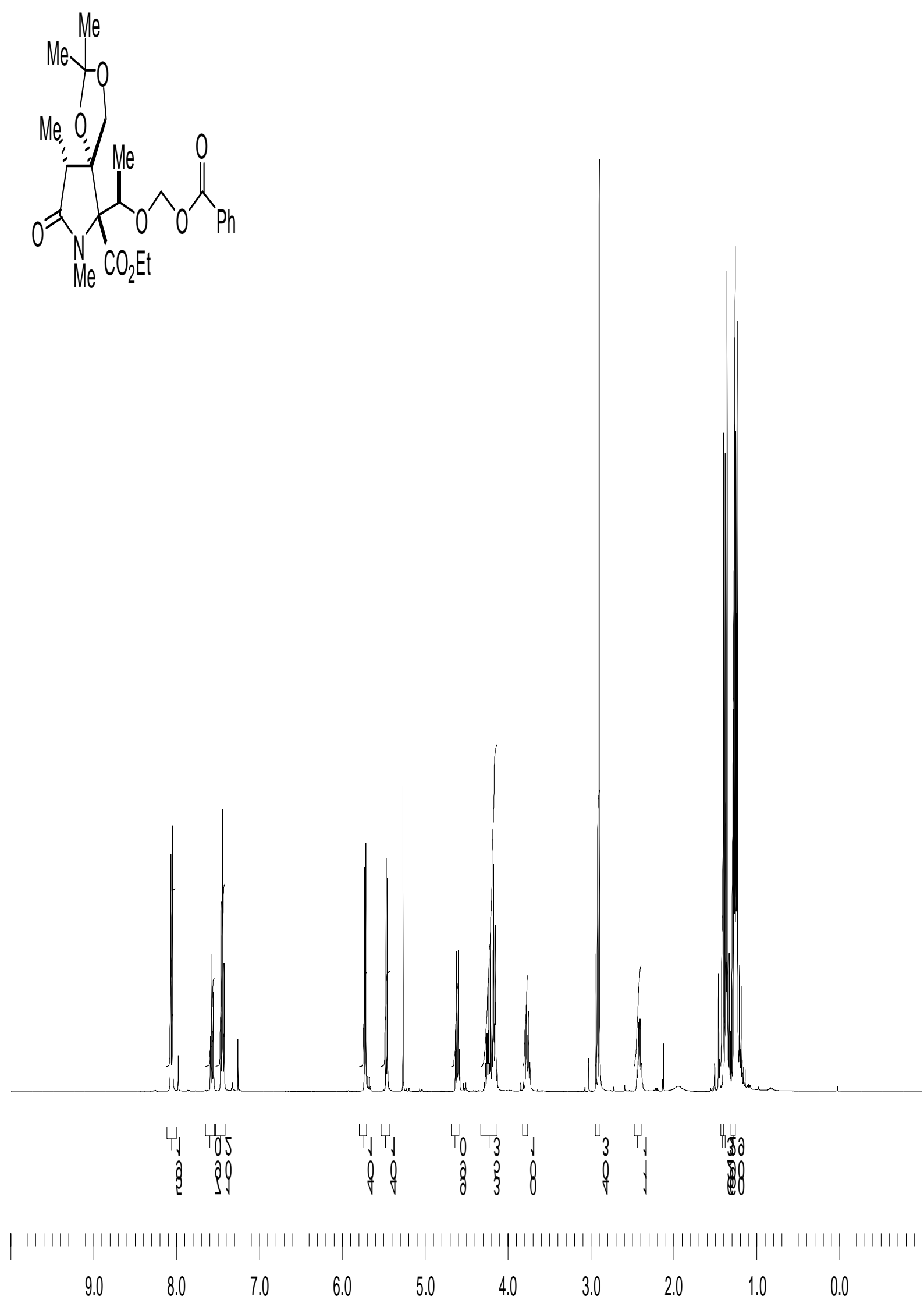
chemical shift (ppm) 


\section{${ }^{13} \mathrm{C}$ NMR of $N$-methylated lactam 19}

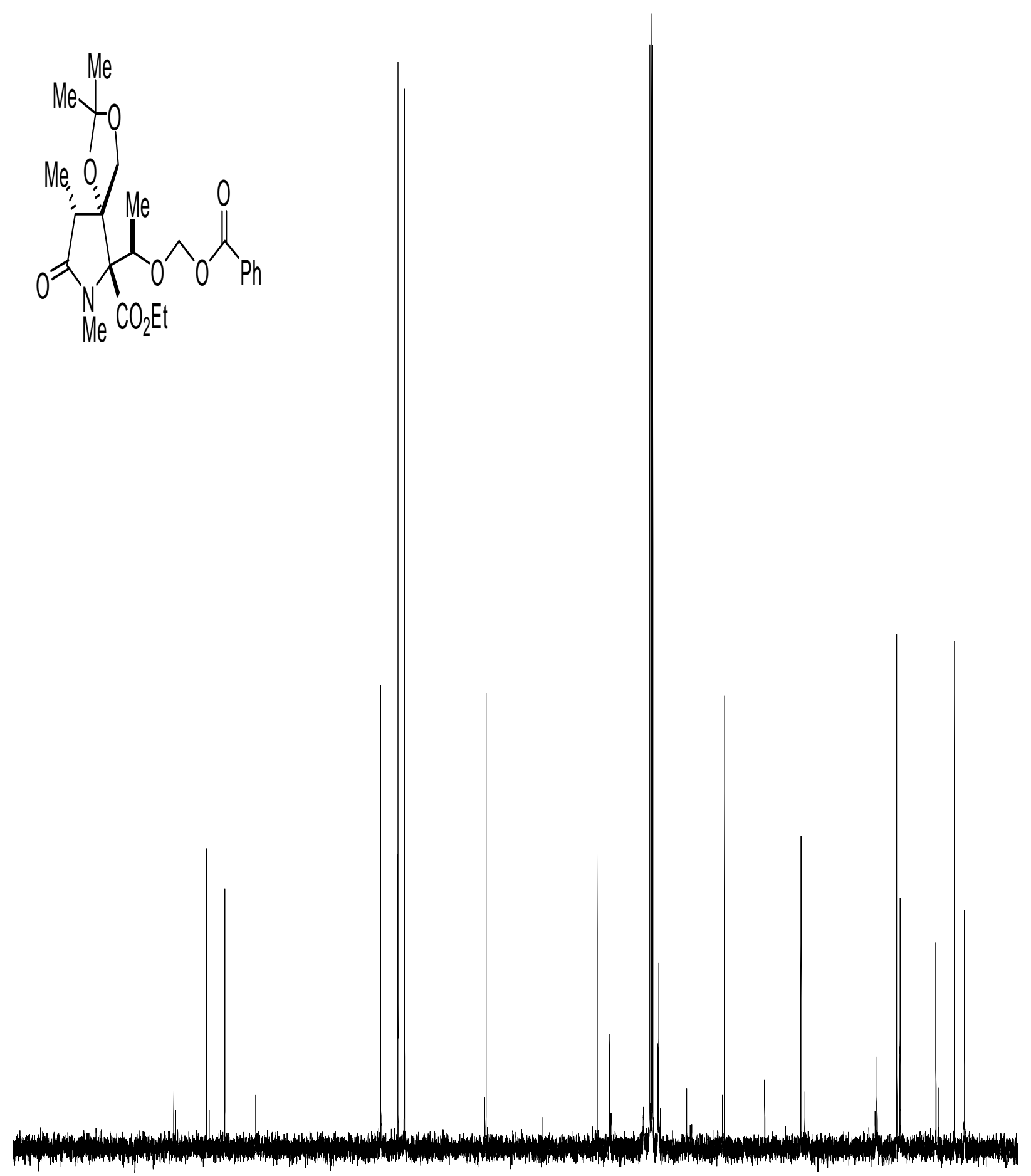

|||||||||||||||||||||||||||||||||||||||||||||||||||||||||||||||||||||||||||||||||||||||||||||||||||||||||||||||||||||||||||||||||||||||||||||||||||||||||||||||||||||||||||||||||||||||||||||||||||||||||||||||||||||||||||||||||

$\begin{array}{llllllllllllllllllll}200 & 190 & 180 & 170 & 160 & 150 & 140 & 130 & 120 & 110 & 100 & 90 & 80 & 70 & 60 & 50 & 40 & 30 & 20 & 10\end{array}$ chemical shift (ppm) 
${ }^{1}$ H NMR of 1,3-diol 20
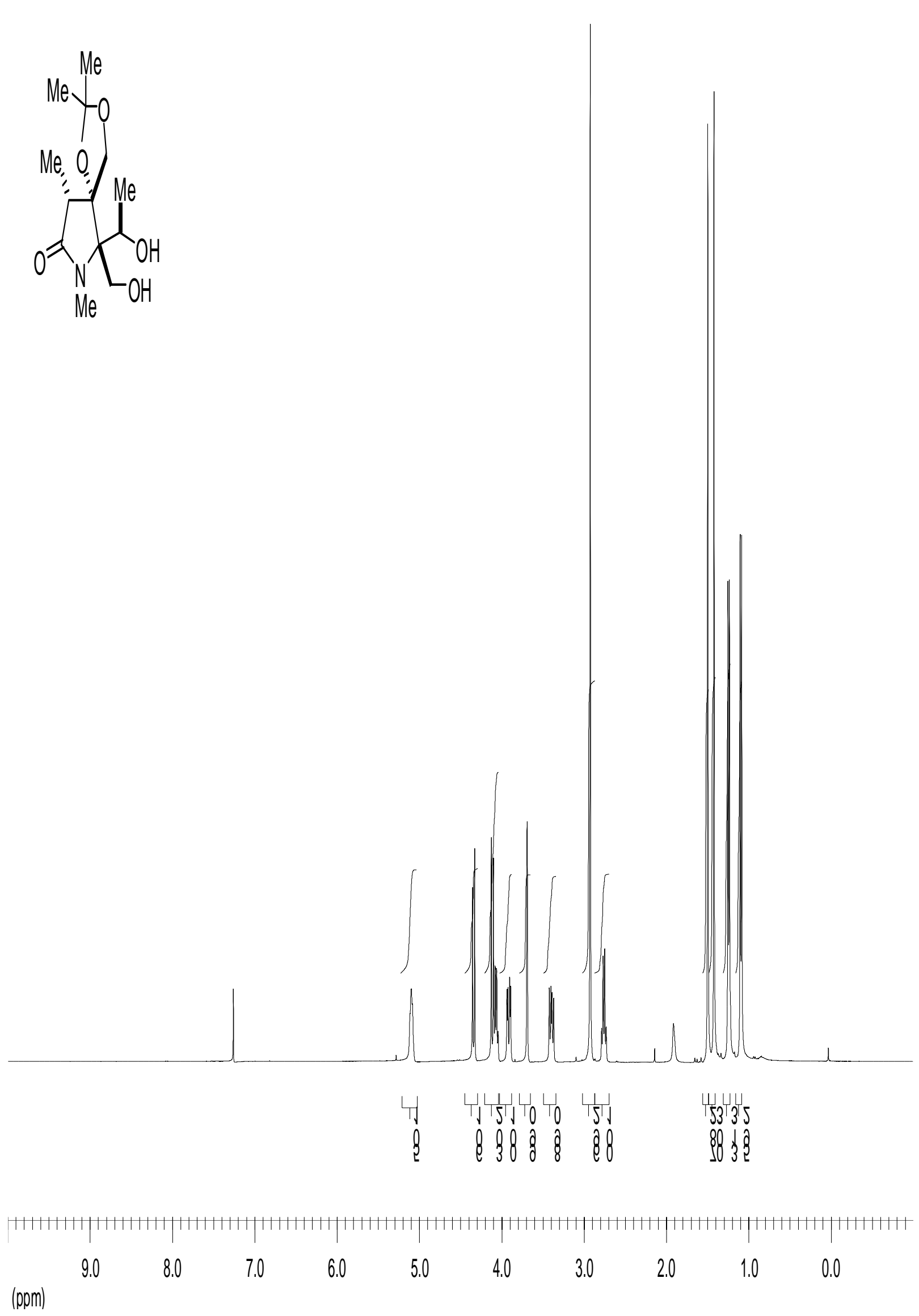
${ }^{13} \mathrm{C}$ NMR of 1,3-diol 20
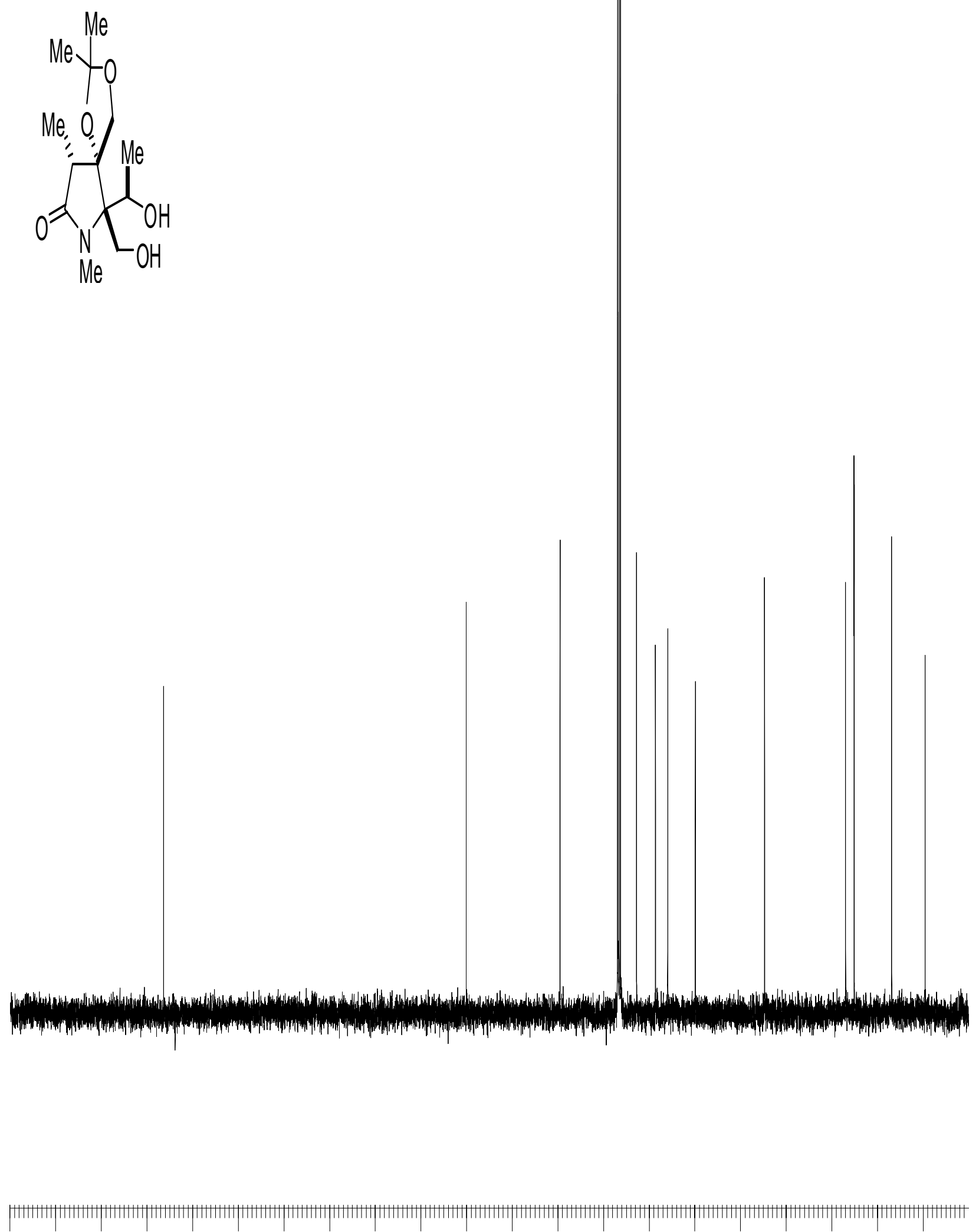

$\begin{array}{llllllllllllllllllll}200 & 190 & 180 & 170 & 160 & 150 & 140 & 130 & 120 & 110 & 100 & 90 & 80 & 70 & 60 & 50 & 40 & 30 & 20 & 10\end{array}$ chemical shitt (ppm) 
${ }^{1} \mathrm{H}$ NMR of hydroxyaldehyde 25
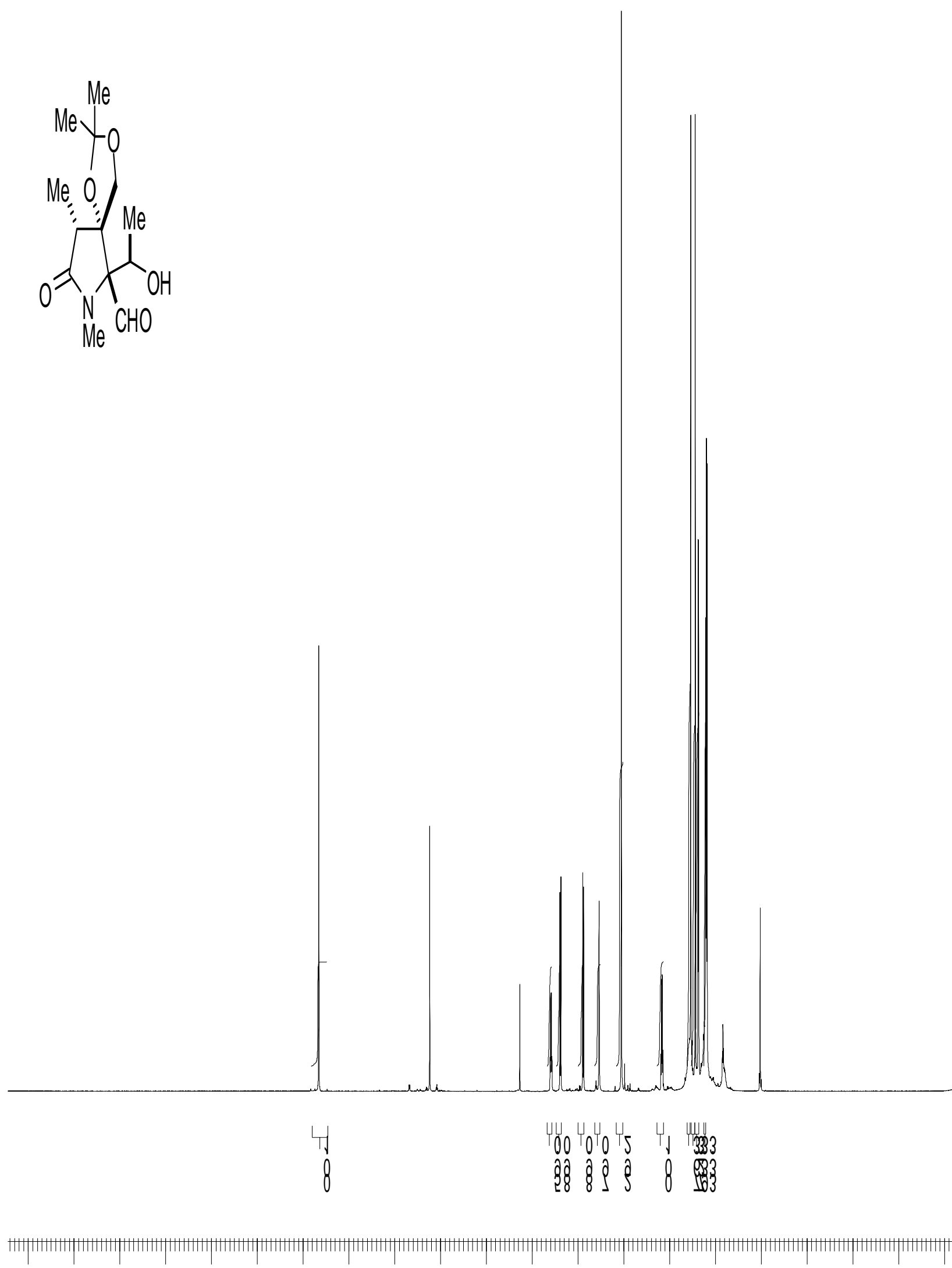

$\begin{array}{llllllllllllllllllll}16.0 & 15.0 & 14.0 & 13.0 & 12.0 & 11.0 & 10.0 & 9.0 & 8.0 & 7.0 & 6.0 & 5.0 & 4.0 & 3.0 & 2.0 & 1.0 & 0.0 & -1.0 & -2.0 & -3.0\end{array}$ chemical shitt (ppm) 


\section{${ }^{13} \mathrm{C}$ NMR of hydroxyaldehyde $\mathbf{2 5}$}
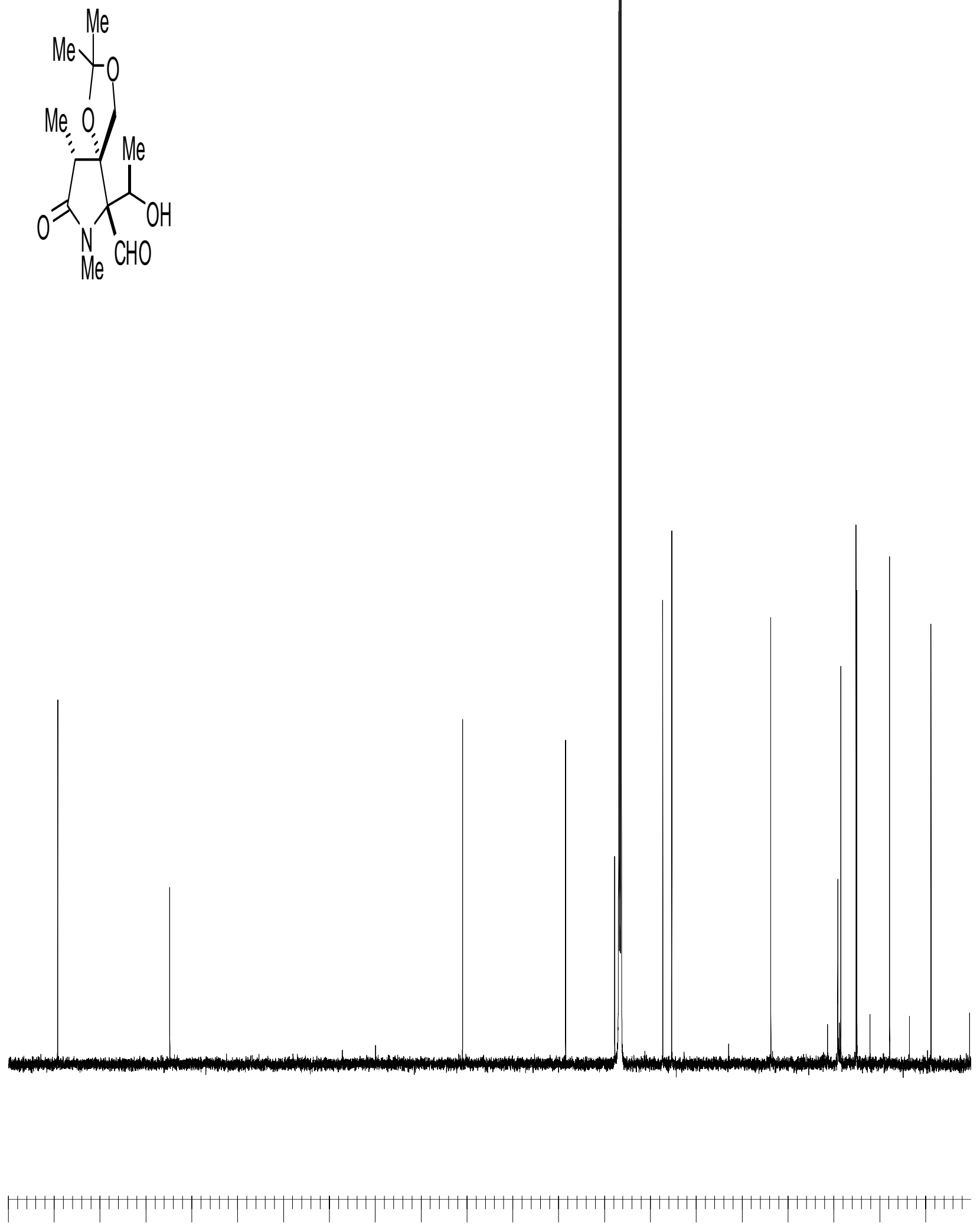

$\begin{array}{llllllllllllllllllll}200 & 190 & 180 & 170 & 160 & 150 & 140 & 130 & 120 & 110 & 100 & 90 & 80 & 70 & 60 & 50 & 40 & 30 & 20 & 10\end{array}$ chemical shift (ppm) 
${ }^{1} \mathrm{H}$ NMR of lactone 3




${ }^{13} \mathrm{C}$ NMR of lactone 3
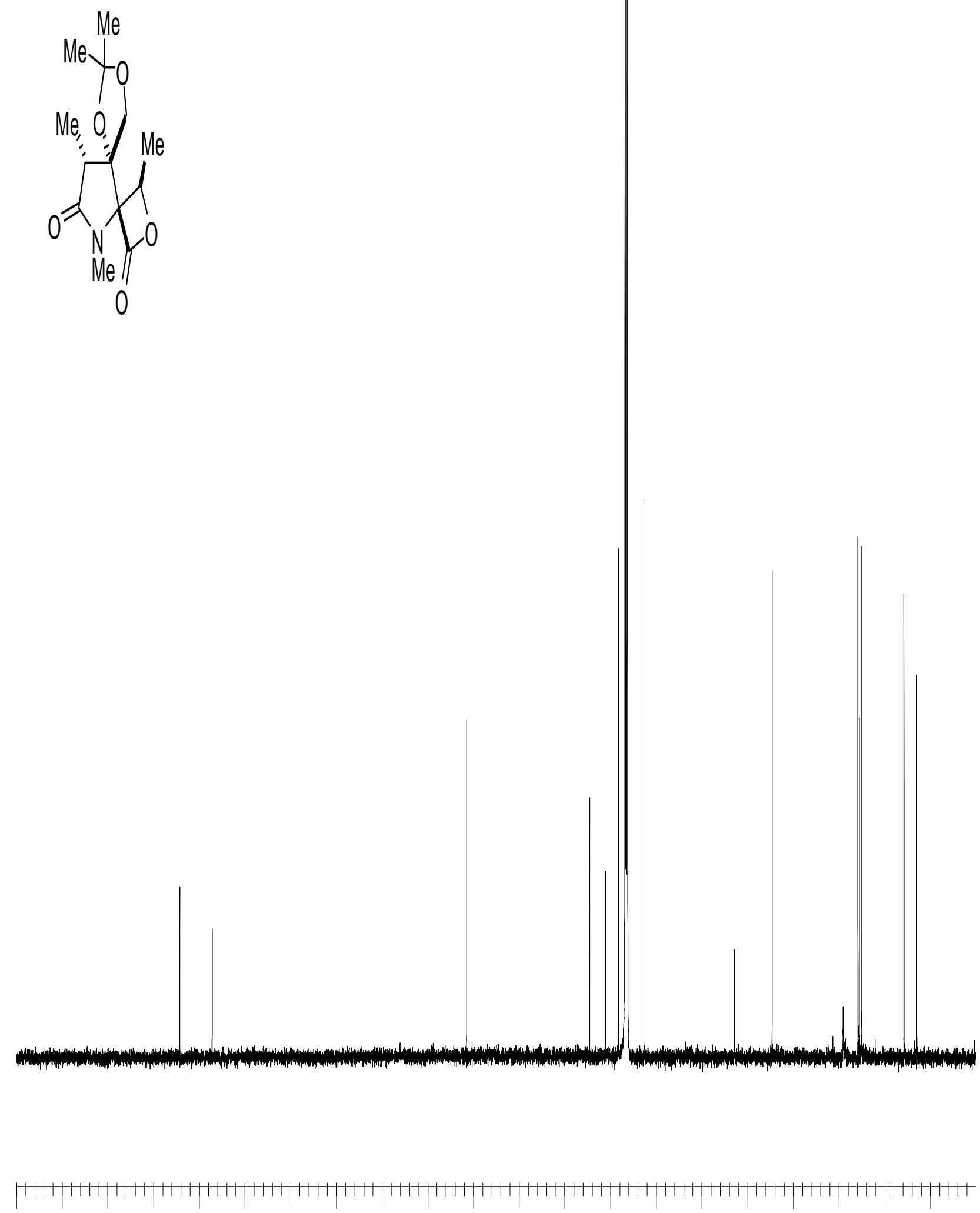

$\begin{array}{llllllllllllllllllll}200 & 190 & 180 & 170 & 160 & 150 & 140 & 130 & 120 & 110 & 100 & 90 & 80 & 70 & 60 & 50 & 40 & 30 & 20 & 10\end{array}$ chemical shift (ppm) 